Ann. Scient. Éc. Norm. Sup.,

$4^{\text {e }}$ série, t. 39, 2006, p. 415 à 466.

\title{
MODULI OF METAPLECTIC BUNDLES ON CURVES AND THETA-SHEAVES
}

\author{
By SERGEY LYSENKO
}

ABSTRACT. - We give a geometric interpretation of the Weil representation of the metaplectic group, placing it in the framework of the geometric Langlands program.

For a smooth projective curve $X$ we introduce an algebraic stack $\widetilde{\operatorname{Bun}}_{G}$ of metaplectic bundles on $X$. It also has a local version $\widetilde{\mathrm{Gr}}_{G}$, which is a gerbe over the affine Grassmanian of $G$. We define a categorical version of the (nonramified) Hecke algebra of the metaplectic group. This is a category $\operatorname{Sph}\left(\widetilde{\mathrm{Gr}}_{G}\right)$ of certain perverse sheaves on $\widetilde{\mathrm{Gr}}_{G}$, which act on $\widetilde{\mathrm{Bun}}_{G}$ by Hecke operators. A version of the Satake equivalence is proved describing $\operatorname{Sph}\left(\widetilde{\mathrm{Gr}}_{G}\right)$ as a tensor category. Further, we construct a perverse sheaf on $\widetilde{\operatorname{Bun}}_{G}$ corresponding to the Weil representation and show that it is a Hecke eigen-sheaf with respect to $\operatorname{Sph}\left(\widetilde{\operatorname{Gr}}_{G}\right)$.

(c) 2006 Elsevier Masson SAS

RÉSUMÉ. - On donne une interprétation géométrique de la représentation de Weil du groupe métaplectique, qui s'inscrit dans le cadre du programme de Langlands géométrique.

Pour une courbe $X$ lisse projective on introduit un champ algébrique $\widetilde{\mathrm{Bun}}_{G}$ des fibrés métaplectiques sur $X$. Il admet aussi une version locale $\widetilde{\mathrm{Gr}}_{G}$, qui est une gerbe sur la grassmanienne affine de $G$. On définit une version catégorique de l'algèbre de Hecke (non ramifiée) du groupe métaplectique. C'est une catégorie $\operatorname{Sph}\left(\widetilde{\mathrm{Gr}}_{G}\right)$ de certains faisceaux pervers sur $\widetilde{\mathrm{Gr}}_{G}$, qui agissent sur $\widetilde{\mathrm{Bun}}_{G}$ par les opérateurs de Hecke. On démontre une version de l'équivalence de Satake qui décrit la catégorie tensorielle $\operatorname{Sph}\left(\widetilde{G r}_{G}\right)$. Ensuite, on construit un faisceau pervers sur $\widetilde{\operatorname{Bun}}_{G}$ qui correspond à la représentation de Weil et on établit sa propriété de Hecke par rapport à $\mathrm{Sph}\left(\widetilde{\mathrm{Gr}}_{G}\right)$.

(c) 2006 Elsevier Masson SAS

\section{Introduction}

1.1. Historically $\theta$-series (such as, in one variable, $\sum q^{n^{2}}$ ) have been one of the major methods of constructing automorphic forms. A representation-theoretic approach to the theory of $\theta$-series, as discovered by A. Weil [23] and extended by R. Howe [15], is based on the oscillator representation of the metaplectic group (cf. [22] for a recent survey). In this paper we propose a geometric interpretation of this representation (in the non-ramified case) placing it in the framework of the geometric Langlands program.

Let $k=\mathbb{F}_{q}$ be a finite field with $q$ odd. Set $K=k((t))$ and $\mathcal{O}=k[[t]]$. Let $\Omega$ denote the completed module of relative differentials of $\mathcal{O}$ over $k$. Let $M$ be a free $\mathcal{O}$-module of rank $2 n$ given with a non-degenerate symplectic form $\bigwedge^{2} M \rightarrow \Omega$. It is known that the continuous cohomology group $\mathrm{H}^{2}(\mathbb{S p}(M)(K),\{ \pm 1\})$ is isomorphic to $\mathbb{Z} / 2 \mathbb{Z}[19,10.4]$. As $\mathbb{S p}(M)(K)$ is 
a perfect group, the corresponding metaplectic extension

$$
1 \rightarrow\{ \pm 1\} \stackrel{i}{\longrightarrow} \widehat{\mathbb{S p}}(M)(K) \rightarrow \mathbb{S p}(M)(K) \rightarrow 1
$$

is unique up to unique isomorphism ${ }^{1}$. It can be constructed in two essentially different ways.

Recall the classical construction of A. Weil [23]. The Heisenberg group is $H(M)=M \oplus \Omega$ with operation

$$
\left(m_{1}, \omega_{1}\right)\left(m_{2}, \omega_{2}\right)=\left(m_{1}+m_{2}, \omega_{1}+\omega_{2}+\frac{1}{2}\left\langle m_{1}, m_{2}\right\rangle\right) .
$$

Fix a prime $\ell$ that does not divide $q$. Let $\psi: k \rightarrow \overline{\mathbb{Q}}_{\ell}^{*}$ be a nontrivial additive character. Let $\chi: \Omega(K) \rightarrow \overline{\mathbb{Q}}_{\ell}$ be given by $\chi(\omega)=\psi(\operatorname{Res} \omega)$. By the Stone and Von Neumann theorem [21], there is a unique (up to isomorphism) smooth irreducible representation $\left(\rho, \mathcal{S}_{\psi}\right)$ of $H(M)(K)$ over $\overline{\mathbb{Q}}_{\ell}$ with central character $\chi$. The group $\operatorname{Sp}(M)$ acts on $H(M)$ by group automorphisms $(m, \omega) \stackrel{g}{\longrightarrow}(g m, \omega)$ This gives rise to the group

$$
\begin{aligned}
\widetilde{\operatorname{Sp}}(M)(K)=\{(g, M[g]) \mid & g \in \mathbb{S p}(M)(K), M[g] \in \operatorname{Aut} \mathcal{S}_{\psi} \\
& \rho(g m, \omega) \circ M[g]=M[g] \circ \rho(m, \omega) \\
& \text { for }(m, \omega) \in H(M)(K)\} .
\end{aligned}
$$

The group $\widetilde{\mathbb{S p}}(M)(K)$ is an extension of $\mathbb{S p}(M)(K)$ by $\overline{\mathbb{Q}}_{\ell}^{*}$. Its commutator subgroup is an extension of $\operatorname{Sp}(M)(K)$ by $\{ \pm 1\} \hookrightarrow \overline{\mathbb{Q}}_{\ell}^{*}$, uniquely isomorphic to (1).

Another way is via Kac-Moody groups. Namely, view $\operatorname{Sp}(M)(K)$ as an ind-scheme over $k$. Let

$$
1 \rightarrow \mathbb{G}_{m} \rightarrow \overline{\mathbb{S p}}(M)(K) \rightarrow \mathbb{S p}(M)(K) \rightarrow 1
$$

denote the canonical extension, here $\overline{\operatorname{Sp}}(M)(K)$ is an ind-scheme over $k$ (cf. [12]). Passing to $k$-points we get an extension of abstract groups $1 \rightarrow k^{*} \rightarrow \overline{\operatorname{Sp}}(M)(K) \rightarrow \mathbb{S p}(M)(K) \rightarrow 1$. Then (1) is the push-forward of this extension under $k^{*} \rightarrow k^{*} /\left(k^{*}\right)^{2}$.

The second construction underlies one of our main results, the Tannakian description of the Langlands dual to the metaplectic group. Namely, the canonical splitting of (2) over $\operatorname{Sp}(M)(\mathcal{O})$ yields a splitting of $(1)$ over $\operatorname{Sp}(M)(\mathcal{O})$. Consider the Hecke algebra

$$
\begin{gathered}
\mathcal{H}=\left\{f: \mathbb{S p}(M)(\mathcal{O}) \backslash \widehat{\mathbb{S p}}(M)(K) / \mathbb{S p}(M)(\mathcal{O}) \rightarrow \overline{\mathbb{Q}}_{\ell} \mid f(i(-1) g)=-f(g), g \in \widehat{\mathbb{S p}}(M)(K) ;\right. \\
f \text { is of compact support }\} .
\end{gathered}
$$

The product is convolution, defined using the Haar measure on $\widehat{\mathbb{S p}}(M)(K)$ for which the inverse image of $\operatorname{Sp}(M)(\mathcal{O})$ has volume 1.

Set $G=\mathbb{S p}(M)$. Let $\check{G}$ denote $\mathbb{S p}_{2 n}$ viewed as an algebraic group over $\overline{\mathbb{Q}}_{\ell}$. Let $\operatorname{Rep}(\check{G})$ denote the category of finite-dimensional representations of $\breve{G}$. Write $K(\operatorname{Rep}(\check{G}))$ for the Grothendieck ring of $\operatorname{Rep}(\breve{G})$ over $\overline{\mathbb{Q}}_{\ell}$. There is a canonical isomorphism of $\overline{\mathbb{Q}}_{\ell}$-algebras

$$
\mathcal{H} \widetilde{\rightarrow} K(\operatorname{Rep}(\check{G})) .
$$

Actually, a categorical version of this isomorphism is proved. Consider the affine Grassmanian $\operatorname{Gr}_{G}=G(K) / G(\mathcal{O})$, viewed as an ind-scheme over $k$. Let $W$ denote the nontrivial $\ell$-adic

\footnotetext{
${ }^{1}$ The notation $\widehat{\operatorname{Sp}}(M)(K)$ is ambiguous, these are not $K$-points of an algebraic group.

$4^{\mathrm{e}}$ SÉRIE - TOME $39-2006-\mathrm{N}^{\circ} 3$
} 
local system of rank one on $\mathbb{G}_{m}$ corresponding to the covering $\mathbb{G}_{m} \rightarrow \mathbb{G}_{m}, x \mapsto x^{2}$. Denote by $\operatorname{Sph}\left(\widetilde{\mathrm{Gr}}_{G}\right)$ the category of $G(\mathcal{O})$-equivariant perverse sheaves on $\bar{G}(K) / G(\mathcal{O})$, which are also $\left(\mathbb{G}_{m}, W\right)$-equivariant. Here $\widetilde{\mathrm{Gr}}_{G}$ denotes the stack quotient of $\bar{G}(K) / G(\mathcal{O})$ by $\mathbb{G}_{m}$ with respect to the action $g \stackrel{x}{\longrightarrow} x^{2} g, x \in \mathbb{G}_{m}, g \in \bar{G}(K)$. Actually, $\operatorname{Sph}\left(\widetilde{\mathrm{Gr}}_{G}\right)$ is a full subcategory of the category of perverse sheaves on $\widetilde{\mathrm{Gr}}_{G}$.

Replacing for simplicity $k$ by an algebraically closed field, we equip $\operatorname{Sph}\left(\widetilde{\operatorname{Gr}}_{G}\right)$ with the structure of a rigid tensor category. We establish a canonical equivalence of tensor categories

$$
\operatorname{Sph}\left(\widetilde{\mathrm{Gr}}_{G}\right) \stackrel{\longrightarrow}{\rightarrow} \operatorname{Rep}(\check{G}) .
$$

1.2. In the global setting let $X$ be a smooth projective curve over $k$. Let $G$ denote the sheaf of automorphisms of $\mathcal{O}_{X}^{n} \oplus \Omega^{n}$ (now $\Omega$ is the canonical line bundle on $X$ ) preserving the symplectic form $\bigwedge^{2}\left(\mathcal{O}_{X}^{n} \oplus \Omega^{n}\right) \rightarrow \Omega$. The stack Bun $G$ of $G$-bundles (= $G$-torsors) on $X$ classifies vector bundles $M$ of rank $2 n$ on $X$, given with a non-degenerate symplectic form $\wedge^{2} M \rightarrow \Omega$. We introduce an algebraic stack $\widetilde{\mathrm{Bun}}_{G}$ of metaplectic bundles on $X$. The stack $\widetilde{\mathrm{Gr}}_{G}$ is a local version of $\widetilde{\mathrm{Bun}}_{G}$. The category $\mathrm{Sph}\left(\widetilde{\mathrm{Gr}}_{G}\right)$ acts on $\mathrm{D}\left(\widetilde{\mathrm{Bun}}_{G}\right)$ by Hecke operators.

We construct a perverse sheaf Aut on $\widehat{\mathrm{Bun}}_{G}$, a geometric analog of the Weil representation. We calculate the fibres of Aut and its constant terms for maximal parabolic subgroups of $G$. Finally, we argue that Aut is a Hecke eigensheaf on $\widetilde{\operatorname{Bun}}_{G}$ with eigenvalue

$$
\mathrm{St}=\mathrm{R} \Gamma\left(\mathbb{P}^{2 n-1}, \overline{\mathbb{Q}}_{\ell}\right) \otimes \overline{\mathbb{Q}}_{\ell}[1]\left(\frac{1}{2}\right)^{\otimes 2 n-1}
$$

viewed as a constant complex on $X$. Note that $\mathrm{St}$ is equipped with an action of $\mathrm{SL}_{2}$ of Arthur, the corresponding representation of $\mathrm{SL}_{2}$ is irreducible of dimension $2 n$ and admits a unique, up to a multiple, symplectic form. One may imagine that Aut corresponds to a group homomorphism $\pi_{1}(X) \times \mathrm{SL}_{2} \rightarrow \check{G}$ trivial on $\pi_{1}(X)$. This agrees with Arthur's conjectures.

\section{Weil representation and motivations}

2.1. Let $X$ be a smooth projective absolutely irreducible curve over $k=\mathbb{F}_{q}, F=\mathbb{F}_{q}(X), \mathbb{A}$ be the adeles rings of $F, \mathcal{O} \subset \mathbb{A}$ be the entire adeles. Assume that $q$ is odd. Fix a prime $\ell$ that does not divide $q$. Let $\Omega$ denote the canonical line bundle on $X$.

Let $M$ be a $2 n$-dimensional vector space over $F$ with symplectic form $\bigwedge^{2} M \rightarrow \Omega_{F}$, where $\Omega_{F}$ is the generic fibre of $\Omega$. The Heisenberg group $H(M)=M \oplus \Omega_{F}$ with operation

$$
\left(m_{1}, \omega_{1}\right)\left(m_{2}, \omega_{2}\right)=\left(m_{1}+m_{2}, \omega_{1}+\omega_{2}+\frac{1}{2}\left\langle m_{1}, m_{2}\right\rangle\right)
$$

is algebraic over $F$. Fix a nontrivial additive character $\psi: \mathbb{F}_{q} \rightarrow \overline{\mathbb{Q}}_{\ell}^{*}$. Then $H(M)(\mathbb{A})=$ $M(\mathbb{A}) \oplus \Omega(\mathbb{A})$ admits a canonical central character $\chi: \Omega(\mathbb{A}) / \Omega(F) \rightarrow \overline{\mathbb{Q}}_{\ell}^{*}$ given by

$$
\chi(\omega)=\psi\left(\sum_{x \in X} \operatorname{tr}_{k(x) / k} \operatorname{Res} \omega_{x}\right) .
$$

The Stone and Von Neumann theorem [21] says that there is a unique (up to isomorphism) smooth irreducible representation $\left(\rho, \mathcal{S}_{\psi}\right)$ of $H(M)(\mathbb{A})$ over $\overline{\mathbb{Q}}_{\ell}$ with central character $\chi$. The 
group $\operatorname{Sp}(M)$ acts on $H(M)$ by group automorphisms $(m, \omega) \stackrel{g}{\longrightarrow}(g m, \omega)$. This defines the global metaplectic group

$$
\begin{aligned}
\widetilde{\mathbb{S p}}(M)(\mathbb{A})=\{(g, M[g]) \mid & g \in \mathbb{S p}(M)(\mathbb{A}), M[g] \in \operatorname{Aut} \mathcal{S}_{\psi}, \\
& \rho(g m, \omega) \circ M[g]=M[g] \circ \rho(m, \omega) \text { for }(m, \omega) \in H(M)(\mathbb{A})\}
\end{aligned}
$$

included into an exact sequence

$$
1 \rightarrow \overline{\mathbb{Q}}_{\ell}^{*} \rightarrow \widetilde{\operatorname{Sp}}(M)(\mathbb{A}) \rightarrow \mathbb{S p}(M)(\mathbb{A}) \rightarrow 1 .
$$

The representation of $\widetilde{\mathbb{S p}}(M)(\mathbb{A})$ on $S_{\psi}$ is called the Weil (or oscillator) representation [23].

For a subgroup $K \subset \mathbb{S p}(M)(\mathbb{A})$ write $\widetilde{K}$ for the preimage of $K$ in $\widetilde{\mathbb{S p}}(M)(\mathbb{A})$. Since $\chi$ is trivial on $\Omega_{F}$, one may talk about $H(M)$-invariant functionals on $S_{\psi}$, they are called thetafunctionals. The space of theta-functionals is 1-dimensional and preserved by $\widetilde{\mathbb{S p}}(M)(F)$, so the action of $\widetilde{\mathbb{S p}}(M)(F)$ on this space defines a splitting of (3) over $\operatorname{Sp}(M)(F)$.

View

$$
\operatorname{Funct}(\mathbb{S p}(M)(F) \backslash \widetilde{\operatorname{Sp}}(M)(\mathbb{A}))=\left\{f: \mathbb{S p}(M)(F) \backslash \widetilde{\mathbb{S p}}(M)(\mathbb{A}) \rightarrow \overline{\mathbb{Q}}_{\ell}\right\}
$$

as a representation of $\widetilde{\mathbb{S p}}(M)(\mathbb{A})$ by right translations. A theta-functional $\Theta: S_{\psi} \rightarrow \overline{\mathbb{Q}}_{\ell}$ defines a morphism of $\widetilde{\mathbb{S p}}(M)(\mathbb{A})$-modules

$$
\mathcal{S}_{\psi} \rightarrow \operatorname{Funct}(\operatorname{Sp}(M)(F) \backslash \widetilde{\mathbb{S p}}(M)(\mathbb{A}))
$$

sending $\phi$ to $\theta_{\phi}$ given by $\theta_{\phi}(g)=\Theta(g \phi)$ for $g \in \widetilde{\mathbb{S p}}(M)(\mathbb{A})$.

Now assume that $M$ is actually a rank $2 n$ vector bundle on $X$ with symplectic form $\bigwedge^{2} M \rightarrow \Omega$. Then we get the subgroups $\operatorname{Sp}(M)(\mathcal{O}) \subset \mathbb{S p}(M)(\mathbb{A})$ and $M(\mathcal{O}) \oplus \Omega(\mathcal{O}) \subset$ $H(M)(\mathbb{A})$. Moreover, the space of $M(\mathcal{O}) \oplus \Omega(\mathcal{O})$-invariants in $\mathcal{S}_{\psi}$ is 1-dimensional and preserved by $\widetilde{\mathbb{S p}}(M)(\mathcal{O})$. The action of $\widetilde{\operatorname{Sp}}(M)(\mathcal{O})$ on this space yields a splitting of (3) over $\mathbb{S p}(M)(\mathcal{O})$. If $\phi_{0} \in \mathcal{S}_{\psi}$ is a nonzero $M(\mathcal{O}) \oplus \Omega(\mathcal{O})$-invariant vector then its image under (4) is the classical theta-function

$$
f_{0}: \mathbb{S p}(M)(F) \backslash \widetilde{\mathbb{S p}}(M)(\mathbb{A}) / \mathbb{S p}(M)(\mathcal{O}) \rightarrow \overline{\mathbb{Q}}_{\ell}
$$

that we are going to geometrize.

Let $G$ denote the sheaf of automorphisms of $M$ preserving the form $\bigwedge^{2} M \rightarrow \Omega$. This is a sheaf of groups (in flat topology) on $X$ locally in Zariski topology isomorphic to $\mathbb{S p}_{2 n}$.

2.2. Assume $M=V \oplus\left(V^{*} \otimes \Omega\right)$ is a direct sum of Lagrangian subbundles, the form being given by the canonical pairing $\langle\cdot, \cdot\rangle$ between $V$ and $V^{*}$. Let

$$
\chi_{V}: V(\mathbb{A}) \oplus \Omega(\mathbb{A}) \rightarrow \overline{\mathbb{Q}}_{\ell}^{*}
$$

denote the character $\chi_{V}(v, \omega)=\chi(\omega)$.

We have the subgroup $V(\mathbb{A}) \subset H(M)(\mathbb{A})$. The space of $V(\mathbb{A})$-invariant functionals on $\mathcal{S}_{\psi}$ is 1-dimensional. A choice of such functional identifies $\mathcal{S}_{\psi}$ with the induced representation of $\left(V(\mathbb{A}) \oplus \Omega(\mathbb{A}), \chi_{V}\right)$ to $H(M)(\mathbb{A})$. The latter identifies with the Schwarz space $\mathcal{S}\left(V^{*} \otimes \Omega(\mathbb{A})\right)$ of locally constant compactly supported $\overline{\mathbb{Q}}_{\ell}$-valued functions on $V^{*} \otimes \Omega(\mathbb{A})$, the corresponding

$4^{\text {e }}$ SÉRIE - TOME $39-2006-\mathrm{N}^{\circ} 3$ 
functional on $\mathcal{S}\left(V^{*} \otimes \Omega(\mathbb{A})\right)$ becomes the evaluation at zero $e v: \mathcal{S}\left(V^{*} \otimes \Omega(\mathbb{A})\right) \rightarrow \overline{\mathbb{Q}}_{\ell}$. This is the Schrödinger model of $\mathcal{S}_{\psi}$.

Write $g \in \mathbb{S p}(M)(\mathbb{A})$ as a matrix

$$
g=\left(\begin{array}{ll}
a & b \\
c & d
\end{array}\right)
$$

with $a \in \operatorname{End}(V)(\mathbb{A}), b \in \operatorname{Hom}\left(V^{*} \otimes \Omega, V\right)(\mathbb{A}), d \in \operatorname{End}\left(V^{*}\right)(\mathbb{A}), c \in \operatorname{Hom}\left(V, V^{*} \otimes \Omega\right)(\mathbb{A})$. Write $a^{*}$ for the transpose operator to $a$.

The defined up to a scalar automorphism $M[g]$ of $\mathcal{S}\left(V^{*} \otimes \Omega(\mathbb{A})\right)$ is described as follows.

- For $a \in \mathrm{GL}(V)(\mathbb{A})$ we have $\left(\begin{array}{cc}a & 0 \\ 0 & a^{*-1}\end{array}\right) \in \mathbb{S p}(M)(\mathbb{A})$. Besides, $\left(\begin{array}{ll}1 & b \\ 0 & 1\end{array}\right) \in \mathbb{S p}(M)(\mathbb{A})$ if and only if $b \in\left(V \otimes V \otimes \Omega^{-1}\right)(\mathbb{A})$ is symmetric. For $g$ given by (5) with $c=0$ we have

$$
(M[g] f)\left(v^{*}\right)=\chi\left(\frac{1}{2}\left\langle a^{*} v^{*}, b^{*} v^{*}\right\rangle\right) f\left(a^{*} v^{*}\right), \quad v^{*} \in V^{*} \otimes \Omega(\mathbb{A}),
$$

- if $b: V^{*} \otimes \Omega(\mathbb{A}) \widetilde{\rightarrow} V(\mathbb{A})$ then $g=\left(\begin{array}{rr}0 & b \\ -b^{*-1} & 0\end{array}\right) \in \mathbb{S p}(M)(\mathbb{A})$ and

$$
(M[g] f)\left(v^{*}\right)=\int_{V(\mathbb{A})} \chi\left(\left\langle v, v^{*}\right\rangle\right) f\left(b^{-1} v\right) \mathrm{d} v, \quad v^{*} \in V^{*} \otimes \Omega(\mathbb{A})
$$

for any Haar measure $d v$ on $V(\mathbb{A})$.

Let $P \subset G$ denote the Siegel parabolic subgroup preserving $V$. The subgroup $\widetilde{P}(\mathbb{A})$ preserves $e v$ up to a multiple, so defining a splitting of $(3)$ over $P(\mathbb{A})$. This splitting coincides with the one given by (6).

Let $\phi_{0} \in \mathcal{S}\left(V^{*} \otimes \Omega(\mathbb{A})\right)$ denote the characteristic function of $V^{*} \otimes \Omega(\mathcal{O})$. Using (6) and (7) one shows that $\phi_{0}$ generates the space of $\operatorname{Sp}(M)(\mathcal{O})$-invariants in $\mathcal{S}\left(V^{*} \otimes \Omega(\mathbb{A})\right)$. In this model of $\mathcal{S}_{\psi}$ the theta functional $\Theta: \mathcal{S}\left(V^{*} \otimes \Omega(\mathbb{A})\right) \rightarrow \overline{\mathbb{Q}}_{\ell}$ is given by

$$
\Theta(\phi)=\sum_{v^{*} \in V^{*} \otimes \Omega(F)} \phi\left(v^{*}\right) \quad \text { for } \phi \in \mathcal{S}\left(V^{*} \otimes \Omega(\mathbb{A})\right) .
$$

Let $f_{0}$ denote the image of $\phi_{0}$ under the corresponding map (4). Let us calculate the composition

$$
P(F) \backslash P(\mathbb{A}) / P(\mathcal{O}) \rightarrow \mathbb{S p}(M)(F) \backslash \widetilde{\mathbb{S p}}(M)(\mathbb{A}) / \mathbb{S p}(M)(\mathcal{O}) \stackrel{f_{0}}{\longrightarrow} \overline{\mathbb{Q}}_{\ell}
$$

denoted by $f_{P}$. We used the fact that the splittings of (3) over $P(\mathbb{A})$ and $\operatorname{Sp}(M)(\mathcal{O})$ are compatible over $P(\mathcal{O})$.

Denote by $\mathrm{Bun}_{n}$ the $k$-stack of rank $n$ vector bundles on $X$. The set $\mathrm{GL}(V)(\mathbb{A}) / \mathrm{GL}(V)(\mathcal{O})$ naturally identifies with the isomorphism classes of pairs $(L, \alpha)$, where $L \in \operatorname{Bun}_{n}(k)$ and $\alpha: L(F) \underset{\rightarrow}{\longrightarrow} V(F)$. Here $L(F)$ is the generic fibre of $L$.

Let $a \in \mathrm{GL}(V)(\mathbb{A})$ and $(L, \alpha)$ be the pair attached to $a \mathrm{GL}(V)(\mathcal{O})$. Then

$$
\left\{v^{*} \in V^{*} \otimes \Omega(F) \mid a^{*} v^{*} \in V^{*} \otimes \Omega(\mathcal{O})\right\} \stackrel{\alpha^{*}}{\longrightarrow} \operatorname{Hom}(L, \Omega)
$$

is an isomorphism.

The group $P$ fits into an exact sequence $1 \rightarrow\left(\mathrm{Sym}^{2} V\right) \otimes \Omega^{-1} \rightarrow P \rightarrow \mathrm{GL}(V) \rightarrow 1$ of algebraic groups over $X$. For $g \in P(\mathbb{A})$ we get 


$$
\begin{aligned}
f_{P}(g) & =\Theta\left(g \phi_{0}\right)=\sum_{v^{*} \in V^{*} \otimes \Omega(F)}\left(g \phi_{0}\right)\left(v^{*}\right) \\
& =\sum_{v^{*} \in V^{*} \otimes \Omega(F)} \chi\left(\frac{1}{2}\left\langle a^{*} v^{*}, b^{*} v^{*}\right\rangle\right) \phi_{0}\left(a^{*} v^{*}\right) \\
& =\sum_{s \in \operatorname{Hom}(L, \Omega)} \chi\left(\frac{1}{2}\left\langle s, a b^{*} s\right\rangle\right)
\end{aligned}
$$

in view of (8).

Let $\operatorname{Bun}_{P}$ be the $k$-stack of $P$-bundles on $X$. Its $Y$-points for a scheme $Y$ is the category of $(Y \times X) \times{ }_{X} P$-torsors over $Y \times X$. Then $\operatorname{Bun}_{P}$ classifies pairs $L \in \mathrm{Bun}_{n}$ together with an exact sequence on $X$

$$
0 \rightarrow \mathrm{Sym}^{2} L \rightarrow ? \rightarrow \Omega \rightarrow 0 .
$$

(More generally, for a semidirect product of group schemes $1 \rightarrow U \rightarrow P \rightarrow M \rightarrow 1$ providing a $P$-torsor $\mathcal{F}_{P}$ is equivalent to providing a $M$-torsor $\mathcal{F}_{M}$ and a $U_{\mathcal{F}_{M}}$-torsor of isomorphisms $\operatorname{Isom}\left(\mathcal{F}_{P}, \mathcal{F}_{M} \times_{M} P\right)$ inducing a given one on the corresponding $M$-torsors.)

In view of the bijection $P(F) \backslash P(\mathbb{A}) / P(\mathcal{O}) \stackrel{\sim}{\rightarrow} \operatorname{Bun}_{P}(k)$, the function $f_{P}$ on $\operatorname{Bun}_{P}(k)$ is described as follows. Let a $P$-torsor $\mathcal{F}_{P} \in \operatorname{Bun}_{P}(k)$ be given by $L \in \operatorname{Bun}_{n}(k)$ together with (9). Consider the map $q^{\mathcal{F}_{P}}: \operatorname{Hom}(L, \Omega) \rightarrow k$ sending $s \in \operatorname{Hom}(L, \Omega)$ to the pairing of

$$
s \otimes s \in \operatorname{Hom}\left(\operatorname{Sym}^{2} L, \Omega^{\otimes 2}\right)
$$

with the exact sequence (9). Then

$$
f_{P}\left(\mathcal{F}_{P}\right)=\sum_{s \in \operatorname{Hom}(L, \Omega)} \psi\left(q^{\mathcal{F}_{P}}(s)\right)
$$

The function $f_{P}: \operatorname{Bun}_{P}(k) \rightarrow \overline{\mathbb{Q}}_{\ell}$ is the trace of Frobenius of the following $\ell$-adic complex $S_{P, \psi}$ on $\operatorname{Bun}_{P}$.

Let $p: \mathcal{X} \rightarrow \operatorname{Bun}_{P}$ be the stack over $\operatorname{Bun}_{P}$ with fibre $\operatorname{Hom}(L, \Omega)$. Let $q: \mathcal{X} \rightarrow \mathbb{A}^{1}$ be the map sending $s \in \operatorname{Hom}(L, \Omega)$ to the pairing of (9) with

$$
s \otimes s \in \operatorname{Hom}\left(\operatorname{Sym}^{2} L, \Omega^{\otimes 2}\right) .
$$

The geometric analog of $f_{P}$ is the complex $S_{P, \psi}=p_{!} q^{*} \mathcal{L}_{\psi} \otimes \overline{\mathbb{Q}}_{\ell}[1]\left(\frac{1}{2}\right)^{\otimes \operatorname{dim} \mathcal{X}}$ on $\operatorname{Bun}_{P}$, here $\operatorname{dim} \mathcal{X}$ denotes the dimension of the corresponding connected component of $\mathcal{X}$.

\section{Main results}

\subsection{Notation}

From now on $k$ denotes an algebraically closed field of characteristic $p>2$, all the schemes (or stacks) we consider are defined over $k$.

Let $X$ be a smooth projective connected curve. Write $\Omega$ for the canonical line bundle on $X$. Fix a prime $\ell \neq p$. For a scheme (or stack) $S$ write $\mathrm{D}(S)$ for the bounded derived category of $\ell$-adic étale sheaves on $S$, and $\mathrm{P}(S) \subset \mathrm{D}(S)$ for the category of perverse sheaves (the middle perversity function is always taken in absolute sense over $\operatorname{Spec} k$ ).

$4^{\text {e }}$ SÉRIE - TOME $39-2006-\mathrm{N}^{\circ} 3$ 
Fix a nontrivial character $\psi: \mathbb{F}_{p} \rightarrow \overline{\mathbb{Q}}_{\ell}^{*}$ and denote by $\mathcal{L}_{\psi}$ the corresponding Artin-Shreier sheaf on $\mathbb{A}^{1}$. Fix a square root $\overline{\mathbb{Q}}_{\ell}\left(\frac{1}{2}\right)$ of the sheaf $\overline{\mathbb{Q}}_{\ell}(1)$ on $\operatorname{Spec} \mathbb{F}_{q}$. Isomorphism classes of such correspond to square roots of $q$ in $\overline{\mathbb{Q}}_{\ell}$. Fix an inclusion of fields $\mathbb{F}_{q} \hookrightarrow k$.

If $V \rightarrow S$ and $V^{*} \rightarrow S$ are dual rank $n$ vector bundles over a stack $S$, we normalize the Fourier transform $\operatorname{Four}_{\psi}: \mathrm{D}(V) \rightarrow \mathrm{D}\left(V^{*}\right)$ by $\operatorname{Four}_{\psi}(K)=\left(p_{V^{*}}\right) !\left(\xi^{*} \mathcal{L}_{\psi} \otimes p_{V}^{*} K\right)[n]\left(\frac{n}{2}\right)$, where $p_{V}, p_{V^{*}}$ are the projections, and $\xi: V \times{ }_{S} V^{*} \rightarrow \mathbb{A}^{1}$ is the pairing.

A $G$-torsor on a scheme $S$ is also referred to as a $G$-bundle on $S$. Write Vect ${ }^{\varepsilon}$ for the tensor category of $\mathbb{Z} / 2 \mathbb{Z}$-graded vector spaces, our conventions about this category are those of [8]. Write Vect $\subset \operatorname{Vect}^{\varepsilon}$ for its even component, i.e., the tensor category of vector spaces.

3.1.1. The sheaf (in flat topology) on the category of $k$-schemes represented by $\mu_{2}:=$ $\operatorname{Ker}\left(x \mapsto x^{2}: \mathbb{G}_{m} \rightarrow \mathbb{G}_{m}\right)$ is the constant sheaf $\{ \pm 1\}$.

For a scheme $S$ and a line bundle $\mathcal{A}$ on $S$ denote by $\widetilde{S}$ the following $\mu_{2}$-gerbe over $S$. For an $S$-scheme $S^{\prime}$, the category of $S^{\prime}$-points of $\widetilde{S}$ is the category of pairs $\left(\mathcal{B},\left.\mathcal{B}^{2} \underset{\rightarrow}{\rightarrow}\right|_{S^{\prime}}\right)$, where $\mathcal{B}$ is a line bundle on $S^{\prime}$. Note that $\widetilde{S} \rightarrow S$ is étale.

If $\widetilde{S} \rightarrow S$ admits a section given by invertible $\mathcal{O}_{S}$-module $\mathcal{B}_{0}$ together with $\mathcal{B}_{0}^{2} \widetilde{\mathcal{A}}$ then the gerbe is trivial, that is, $\widetilde{S} \widetilde{\rightrightarrows} B\left(\mu_{2} / S\right)$ over $S$. In this case we get the $S_{2}$-covering $\operatorname{Cov}(\widetilde{S}) \rightarrow \widetilde{S}$, whose fibre consists of isomorphisms $\mathcal{B} \widetilde{\rightarrow} \mathcal{B}_{0}$ whose square is the given one $\mathcal{B}^{2} \breve{\rightarrow}$. This covering is locally trivial in étale topology, but not trivial even for $S=\operatorname{Spec} k$. Actually $S=\operatorname{Cov}(\widetilde{S})$.

3.1.2. If in addition $\mathcal{A}$ is a $\mathbb{Z} / 2 \mathbb{Z}$-graded line bundle on $S$ purely of degree zero, then by definition $\widetilde{S}$ classifies a $\mathbb{Z} / 2 \mathbb{Z}$-graded line bundle $\mathcal{B}$ purely of degree zero, given with a $\mathbb{Z} / 2 \mathbb{Z}$ graded isomorphism $\mathcal{B}^{2} \stackrel{\mathcal{A}}{\rightarrow}$. If $\mathcal{B}$ is a $\mathbb{Z} / 2 \mathbb{Z}$-graded line bundle on $S$ of pure degree (that is, placed in one degree only over each connected component) then a $\mathbb{Z} / 2 \mathbb{Z}$-graded isomorphism $\mathcal{B}^{2} \widetilde{\mathcal{A}}$ yields a (uniquely defined) section of $\widetilde{S}$.

3.2. Let $\operatorname{Bun}_{n}$ be the stack of rank $n$ vector bundles on $X$. Let $G$ denote the sheaf of automorphisms of $\mathcal{O}_{X}^{n} \oplus \Omega^{n}$ preserving the symplectic form $\bigwedge^{2}\left(\mathcal{O}_{X}^{n} \oplus \Omega^{n}\right) \rightarrow \Omega$. So, $G$ is a sheaf of groups in flat topology on the category of $X$-schemes.

The stack $\operatorname{Bun}_{G}$ of $G$-bundles on $X$ classifies $M \in \operatorname{Bun}_{2 n}$ together with a symplectic form $\bigwedge^{2} M \rightarrow \Omega$. A theta-characteristic is a line bundle $\mathcal{N}$ on $X$ equipped with $\mathcal{N} \otimes 2 \stackrel{\Im}{\rightarrow} \Omega$. A choice of a theta-characteristic yields an isomorphism $\operatorname{Bun}_{G} \underset{\rightarrow}{\rightarrow} \mathrm{Bun}_{\mathbb{S p}_{2 n}}$. So, Bun ${ }_{G}$ is a smooth algebraic stack locally of finite type over $k$. Since $\mathbb{S p}_{2 n}$ is simply-connected, Bun ${ }_{G}$ is irreducible [11, Proposition 5]. Let $d_{G}=\operatorname{dimBun} \operatorname{Bun}_{G}=(g-1) \operatorname{dim} \mathfrak{s p}_{2 n}$. To express the dependence on $n$ we write $G_{n}, \operatorname{Bun}_{G_{n}}, d_{G_{n}}$ and so on.

Denote by $\mathcal{A}$ the line bundle on $\operatorname{Bun}_{G}$ whose fibre at $M$ is $\operatorname{det} \mathrm{R} \Gamma(X, M)$ (cf. [8]). As $\chi(M)=0$, we view $\mathcal{A}$ as a $\mathbb{Z} / 2 \mathbb{Z}$-graded line bundle placed in degree zero. It yields a $\mu_{2}$-gerbe

$$
\mathfrak{r}: \widetilde{\operatorname{Bun}}_{G} \rightarrow \operatorname{Bun}_{G}
$$

So, $S$-points of $\widetilde{\operatorname{Bun}}_{G}$ is the category: a line bundle $\mathcal{B}$ on $S$, a vector bundle $M$ on $S \times X$ of rank $2 n$ with symplectic form $\bigwedge^{2} M \rightarrow \Omega_{S \times X / S}$, and an isomorphism of $\mathcal{O}_{S}$-modules $\mathcal{B}^{2} \stackrel{\operatorname{det}}{\mathrm{R} \Gamma}(X, M)$.

The idea of using the determinant of cohomology was communicated to me by G. Laumon and goes back to P. Deligne [9].

Let ${ }_{i} \operatorname{Bun}_{G} \hookrightarrow \operatorname{Bun}_{G}$ be the locally closed substack given by $\operatorname{dim} H^{0}(X, M)=i$. Let $\widetilde{\operatorname{Bun}}_{G}$ denote the preimage of ${ }_{i} \operatorname{Bun}_{G}$ under $\mathfrak{r}$. 
LEMmA 1. - Each stratum ${ }_{i} \operatorname{Bun}_{G}$ of $\operatorname{Bun}_{G}$ is nonempty.

Proof. - For $n=1$ take $M=\mathcal{A}(D) \oplus\left(\mathcal{A}^{*} \otimes \Omega(-D)\right)$, where $D$ is an effective divisor of degree $i$ on $X$, and $\mathcal{A}$ is a line bundle on $X$ of degree $g-1$ such that $\mathrm{H}^{0}(X, \mathcal{A})=\mathrm{H}^{1}(X, \mathcal{A})=0$. Such $\mathcal{A}$ exist, because $\operatorname{dim} X^{(g-1)}=g-1$, and the dimension of the Picard scheme of $X$ is $g$. Then $\operatorname{dim} \mathrm{H}^{0}(X, M)=i$.

For any $n$ construct $M \in{ }_{i} \operatorname{Bun}_{G}$ as $M=M_{1} \oplus \cdots \oplus M_{n}$ with $M_{j} \in i_{i_{j}} \operatorname{Bun}_{G_{1}}$ for some $i_{1}+\cdots+i_{n}=i$.

We have a line bundle ${ }_{i} \mathcal{B}$ on ${ }_{i} \operatorname{Bun}_{G}$ whose fibre at $M \in \operatorname{Bun}_{G}$ is $\operatorname{det} \mathrm{H}^{0}(X, M)$. View it as a $\mathbb{Z} / 2 \mathbb{Z}$-graded placed in degree $\operatorname{dim} \mathrm{H}^{0}(X, M)$ modulo 2 . Then for each $i$ we get a $\mathbb{Z} / 2 \mathbb{Z}$-graded isomorphism $\left.{ }_{i} \mathcal{B}^{2} \widetilde{\rightarrow} \mathcal{A}\right|_{i} \operatorname{Bun}_{G}$. By 3.1.2, the gerbe ${ }_{i} \widetilde{\operatorname{Bun}}_{G} \rightarrow{ }_{i} \operatorname{Bun}_{G}$ is trivial. So, we have the two-sheeted covering

$$
{ }_{i} \rho: \operatorname{Cov}\left({ }_{i} \widetilde{\operatorname{Bun}}_{G}\right) \rightarrow{ }_{i} \widetilde{\operatorname{Bun}}_{G} .
$$

By [12, Theorem 17], $\mathcal{A}$ generates the Picard group $\operatorname{Pic}\left(\operatorname{Bun}_{G}\right) \widetilde{\longrightarrow}$. So, the gerb $\mathfrak{r}$ is nontrivial, and the line bundles ${ }_{i} \mathcal{B}$ (viewed as ungraded) do not glue into a line bundle over $\operatorname{Bun}_{G}$.

Definition 1. - For each $i$ define a local system ${ }_{i}$ Aut on ${ }_{i} \widetilde{\operatorname{Bun}}_{G}$ by

$$
{ }_{i} \text { Aut }=\operatorname{Hom}_{S_{2}}\left(\operatorname{sign},{ }_{i} \rho ! \overline{\mathbb{Q}}_{\ell}\right) .
$$

Let $\mathrm{Aut}_{g} \in \mathrm{P}\left(\widetilde{\operatorname{Bun}}_{G}\right)$ (respectively, Aut ${ }_{s} \in \mathrm{P}\left(\widetilde{\operatorname{Bun}}_{G}\right)$ ) denote the Goresky-MacPherson extension of ${ }_{0}$ Aut $\otimes \overline{\mathbb{Q}}_{\ell}\left[d_{G}\right]\left(\frac{d_{G}}{2}\right)$ (respectively, of ${ }_{1}$ Aut $\otimes \overline{\mathbb{Q}}_{\ell}\left[d_{G}-1\right]\left(\frac{d_{G}-1}{2}\right)$ ) under ${ }_{i} \widetilde{\operatorname{Bun}}_{G} \hookrightarrow \widetilde{\operatorname{Bun}}_{G} \cdot{ }^{2}$ Set

$$
\text { Aut }=\operatorname{Aut}_{g} \oplus \text { Aut }_{s} .
$$

By construction, $\mathbb{D}($ Aut $) \stackrel{\Im}{\hookrightarrow}$ Aut canonically.

Here is our main result.

THEOREM 1. - For each $i$ the $*$-restriction Aut $\left.\right|_{i \operatorname{Bun}_{G}}$ identifies with

$$
\text { Aut }\left.\right|_{i \widetilde{\operatorname{Bun}}_{G}} \underset{\rightarrow}{\rightarrow} \text { Aut } \otimes \overline{\mathbb{Q}}_{\ell}[1]\left(\frac{1}{2}\right)^{\otimes d_{G}-i}
$$

(once $\sqrt{-1} \in k$ is fixed, the corresponding isomorphism is well-defined up to a sign). The *-restriction of $\mathrm{Aut}_{g}$ (respectively, of $\mathrm{Aut}_{s}$ ) to ${ }_{i} \widetilde{\operatorname{Bun}}_{G}$ vanishes for $i$ odd (respectively, even).

Remark 1. - Classically, for two symplectic spaces $W, W^{\prime}$ there is a natural map $\widetilde{\mathbb{S p}}(W) \times$ $\widetilde{\mathbb{S p}}\left(W^{\prime}\right) \rightarrow \widetilde{\mathbb{S p}}\left(W \oplus W^{\prime}\right)$, and the restriction of the metaplectic representation under this map is the tensor product of metaplectic representations of the factors [22, Remark 2.7].

In geometric setting we have a map $s_{n, m}: \operatorname{Bun}_{G_{n}} \times \operatorname{Bun}_{G_{m}} \rightarrow \operatorname{Bun}_{G_{n+m}}$ sending $M, M^{\prime}$ to $M \oplus M^{\prime}$. It extends to a map

$$
\tilde{s}_{n, m}: \widetilde{\operatorname{Bun}}_{G_{n}} \times \widetilde{\operatorname{Bun}}_{G_{m}} \rightarrow \widetilde{\operatorname{Bun}}_{G_{n+m}}
$$

\footnotetext{
${ }^{2}$ Here 'g' stands for generic and 's' for special. We postpone to Proposition 7 the proof of the fact that ${ }_{1}$ Aut is a shifted perverse sheaf on ${ }_{1} \widehat{\operatorname{Bun}}_{G}$.

$4^{\mathrm{e}}$ SÉRIE - TOME $39-2006-\mathrm{N}^{\circ} 3$
} 


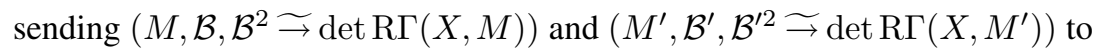

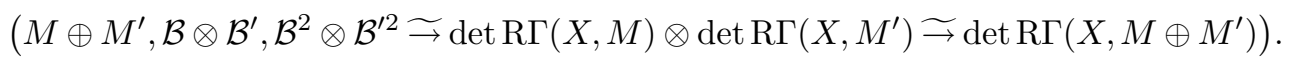

The restriction yields a map $s_{n, m}:{ }_{i} \operatorname{Bun}_{G_{n}} \times{ }_{j} \operatorname{Bun}_{G_{m}} \rightarrow{ }_{i+j} \operatorname{Bun}_{G_{n+m}}$ and we get canonically $s_{n, m}^{*}(i+j \mathcal{B}) \underset{\rightarrow}{\rightarrow} \mathcal{B} \otimes_{j} \mathcal{B}$. For any $i, j$ this yields an isomorphism

$$
\tilde{s}_{n, m}^{*}\left({ }_{i+j} \text { Aut }\right) \widetilde{\rightarrow}_{i} \text { Aut } \otimes_{j} \text { Aut }
$$

of local systems on $\widetilde{\operatorname{Bun}}_{G_{n}} \times{ }_{j} \widetilde{\operatorname{Bun}}_{G_{m}}$. Thus,

$$
\tilde{s}_{n, m}^{*} \mathrm{Aut}_{g} \otimes \overline{\mathbb{Q}}_{\ell}[1]\left(\frac{1}{2}\right)^{\otimes d_{G_{n}}+d_{G_{m}}-d_{G_{n+m}}} \underset{\rightarrow}{\longrightarrow}\left(\mathrm{Aut}_{g} \otimes \mathrm{Aut}_{g}\right) \oplus\left(\mathrm{Aut}_{s} \otimes \mathrm{Aut}_{s}\right)
$$

and

$$
\tilde{s}_{n, m}^{*} \mathrm{Aut}_{s} \otimes \overline{\mathbb{Q}}_{\ell}[1]\left(\frac{1}{2}\right)^{\otimes d_{G_{n}}+d_{G_{m}}-d_{G_{n+m}}} \underset{\longrightarrow}{\longrightarrow}\left(\mathrm{Aut}_{g} \otimes \mathrm{Aut}_{s}\right) \oplus\left(\mathrm{Aut}_{s} \otimes \mathrm{Aut}_{g}\right)
$$

in the completed Grothendieck group $K\left(\widetilde{\operatorname{Bun}}_{G_{n}} \times \widetilde{\operatorname{Bun}}_{G_{m}}\right.$ ) (the completion is with respect to the filtration given by the codimension of support).

3.3. For $1 \leqslant k \leqslant n$ denote by $\operatorname{Bun}_{P_{k}}$ the stack classifying $M \in \operatorname{Bun}_{G}$ together with an isotropic subbundle $L_{1} \subset M$ of rank $k$. We write $L_{-1} \subset M$ for the orthogonal complement of $L_{1}$, so a point of $\operatorname{Bun}_{P_{k}}$ gives rise to a flag $\left(L_{1} \subset L_{-1} \subset M\right)$, and $L_{-1} / L_{1} \in \operatorname{Bun}_{G_{n-k}}$ naturally.

Write $\nu_{k}: \operatorname{Bun}_{P_{k}} \rightarrow \operatorname{Bun}_{G}$ for the projection. Define the map

$$
\tilde{\nu}_{k}: \widetilde{\operatorname{Bun}}_{G_{n-k}} \times_{\operatorname{Bun}_{G_{n-k}}} \operatorname{Bun}_{P_{k}} \rightarrow \widetilde{\operatorname{Bun}}_{G}
$$

as follows. An $S$-point of the source is given by $\left(L_{1} \subset L_{-1} \subset M\right) \in \operatorname{Bun}_{P_{k}}(S)$ together with a $\left(\mathbb{Z} / 2 \mathbb{Z}\right.$-graded of pure degree zero) invertible $\mathcal{O}_{S}$-module $\mathcal{B}$ and $\mathcal{B}^{2} \widetilde{\longrightarrow} \operatorname{det} \mathrm{R} \Gamma\left(X, L_{-1} / L_{1}\right)$. We have a canonical isomorphism of $\mathbb{Z} / 2 \mathbb{Z}$-graded lines

$$
\operatorname{det} \mathrm{R} \Gamma\left(X, L_{1}\right) \otimes \operatorname{det} \mathrm{R} \Gamma\left(X, L_{-1} / L_{1}\right) \otimes \operatorname{det} \mathrm{R} \Gamma\left(X, L_{1}^{*} \otimes \Omega\right) \widetilde{\rightarrow} \operatorname{det} \mathrm{R} \Gamma(X, M)
$$

The map $\tilde{\nu}_{k}$ sends this point to $M \in \operatorname{Bun}_{G}$ together with an invertible $\mathcal{O}_{S}$-module $\mathcal{B}^{\prime}=\mathcal{B} \otimes \operatorname{det} \mathrm{R} \Gamma\left(X, L_{1}\right)$ and $\mathcal{B}^{\prime 2} \underset{\longrightarrow}{\longrightarrow} \operatorname{det} \mathrm{R} \Gamma(X, M)$ given by (11). Since $\mathcal{B}^{\prime}$ is of pure degree as $\mathbb{Z} / 2 \mathbb{Z}$-graded, the map is well-defined by 3.1.2.

Let $\operatorname{Bun}_{Q_{k}}$ be the stack of collections: an exact sequence $0 \rightarrow L_{1} \rightarrow L_{-1} \rightarrow L_{-1} / L_{1} \rightarrow 0$ of vector bundles on $X$ with $L_{1} \in \mathrm{Bun}_{k}$ and $L_{-1} / L_{1} \in \mathrm{Bun}_{2 n-2 k}$, and a symplectic form $\bigwedge^{2}\left(L_{-1} / L_{1}\right) \rightarrow \Omega$ (thus, $\left.L_{-1} / L_{1} \in \operatorname{Bun}_{G_{n-k}}\right)$.

Let $\eta_{k}: \operatorname{Bun}_{P_{k}} \rightarrow \operatorname{Bun}_{Q_{k}}$ denote the natural projection. Let ${ }^{0} \operatorname{Bun}_{Q_{k}} \subset \operatorname{Bun}_{Q_{k}}$ be the open substack given by $\mathrm{H}^{0}\left(X, \mathrm{Sym}^{2} L_{1}\right)=0$.

THEOREM 2. - For the diagram

$$
\widetilde{\operatorname{Bun}}_{G_{n-k}} \times \operatorname{Bun}_{G_{n-k}} \operatorname{Bun}_{Q_{k}} \stackrel{\operatorname{id} \times \eta_{k}}{\longleftarrow \operatorname{Bun}_{G_{n-k}}} \times_{\operatorname{Bun}_{G_{n-k}}} \operatorname{Bun}_{P_{k}} \stackrel{\tilde{\nu}_{k}}{\longrightarrow} \widetilde{\operatorname{Bun}_{G}}
$$


we have an isomorphism

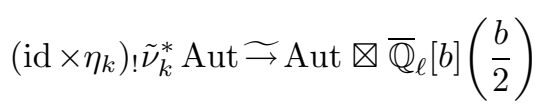

over $\widetilde{\operatorname{Bun}}_{G_{n-k}} \times_{\operatorname{Bun}_{G_{n-k}}}{ }^{0} \operatorname{Bun}_{Q_{k}}$. (Once $\sqrt{-1} \in k$ is fixed, the isomorphism is well-defined up to a sign on generic and special parts.) Here $b\left(L_{1}\right)=d_{G}-d_{G_{n-k}}-\chi\left(L_{1}\right)+2 \chi\left(\Omega^{-1} \otimes \operatorname{Sym}^{2} L_{1}\right)$ is a function of a connected component of ${ }^{0} \mathrm{Bun}_{Q_{k}}$. If $\chi\left(L_{1}\right)$ is even then, over the corresponding connected component, the above isomorphism preserves generic and special parts, otherwise it interchanges them.

3.4. In Section 8.1 we consider the affine Grassmanian $\operatorname{Gr}_{G}$ for $G$, it is equipped with a natural line bundle $\mathcal{L}$ that generates the Picard group of $\mathrm{Gr}_{G}$. Let $\widetilde{G r}_{G} \rightarrow \mathrm{Gr}_{G}$ denote the $\mu_{2}$-gerbe of square roots of $\mathcal{L}$. This is a local version of the gerbe (10). We introduce the category $\operatorname{Sph}\left(\widetilde{\mathrm{Gr}}_{G}\right)^{b}$ of genuine spherical sheaves on $\widetilde{\mathrm{Gr}}_{G}$ (cf. Definitions 4 and 6).

As for usual spherical sheaves on the affine Grassmanian, we equip $\operatorname{Sph}\left(\widetilde{\operatorname{Gr}}_{G}\right)^{b}$ with a structure of a rigid tensor category. Main result of Section 8 is the following version of the Satake equivalence.

THEOREM 3. - The category $\operatorname{Sph}\left(\widetilde{\mathrm{Gr}}_{G}\right)^{b}$ is canonically equivalent, as a tensor category, to the category $\operatorname{Rep}\left(\mathbb{S p}_{2 n}\right)$ of finite-dimensional $\overline{\mathbb{Q}}_{\ell}$-representations of $\mathbb{S p}_{2 n}$.

In Section 9 we define for $K \in \operatorname{Sph}\left(\widetilde{G r}_{G}\right)^{b}$ Hecke operators $\mathrm{H}(K, \cdot): \mathrm{D}\left(\widetilde{\operatorname{Bun}}_{G}\right) \rightarrow$ $\mathrm{D}\left(X \times \widetilde{\operatorname{Bun}}_{G}\right)$ compatible with the tensor structure on $\operatorname{Sph}\left(\widetilde{\mathrm{Gr}}_{G}\right)^{b}$. Finally, we prove Theorem 4 saying that Aut is a Hecke eigen-sheaf with eigenvalue

$$
\mathrm{St}=\mathrm{R} \Gamma\left(\mathbb{P}^{2 n-1}, \overline{\mathbb{Q}}_{\ell}\right) \otimes \overline{\mathbb{Q}}_{\ell}[1]\left(\frac{1}{2}\right)^{\otimes 2 n-1}
$$

viewed as a constant complex on $X$.

Remark 2. - The following observation was communicated to the author by Drinfeld. Let $\sigma$ be the 2-automorphism of id: $\widetilde{\operatorname{Bun}}_{G} \rightarrow \widetilde{\operatorname{Bun}}_{G}$ that acts on $\left(M, \bigwedge^{2} M \rightarrow \Omega, \mathcal{B}^{2} \underset{\rightrightarrows}{\rightarrow} \operatorname{det} \mathrm{R} \Gamma(X, M)\right) \in$ $\widetilde{\operatorname{Bun}}_{G}$ as -1 on $M$ and trivially on $\mathcal{B}$. Then $\sigma$ acts as -1 on Aut ${ }_{s}$ and trivially on Aut $g$. This is a way to think about the decomposition of Aut in a direct sum of perverse shaves.

\section{Finite-dimensional model}

4.1. Let $V$ be a $k$-vector space of dimension $d$. Write $\mathrm{ST}^{2}\left(V^{*}\right)$ for the space of symmetric tensors in $V^{*} \otimes V^{*}$, this is the space of symmetric bilinear forms on $V$. Think of $b \in \operatorname{ST}^{2}\left(V^{*}\right)$ as a map $b: V \rightarrow V^{*}$ such that $b^{*}=b$. Let $p: V \times \mathrm{ST}^{2}\left(V^{*}\right) \rightarrow \mathrm{ST}^{2}\left(V^{*}\right)$ denote the projection. Let $\beta: V \times \mathrm{ST}^{2}\left(V^{*}\right) \rightarrow \mathbb{A}^{1}$ be the map that sends $(v, b)$ to $\langle v, b v\rangle$. Set

$$
S_{\psi}=p_{!} \beta^{*} \mathcal{L}_{\psi} \otimes \overline{\mathbb{Q}}_{\ell}[1]\left(\frac{1}{2}\right)^{\otimes d+\frac{1}{2} d(d+1)} .
$$

Let $\pi: V \rightarrow \operatorname{Sym}^{2} V$ be the map $v \mapsto v \otimes v$. Then

$$
S_{\psi}=\operatorname{Four}_{\psi}\left(\pi ! \overline{\mathbb{Q}}_{\ell}[d]\left(\frac{d}{2}\right)\right)
$$

$4^{e}$ SÉRIE - TOME $39-2006-\mathrm{N}^{\circ} 3$ 
The map $\pi$ is finite, and $\pi ! \overline{\mathbb{Q}}_{\ell}=\mathcal{L}_{0}+\mathcal{L}_{1}$, where $\mathcal{L}_{0}$ is the constant sheaf on the image $\operatorname{Im} \pi$ of $\pi$, and $\mathcal{L}_{1}$ is a nontrivial local system of rank one on $\operatorname{Im} \pi-\{0\}$ extended by zero to $\operatorname{Im} \pi$. So, $S_{\psi}$ is a direct sum of two irreducible perverse sheaves.

LEMMA 2. $-S_{\psi}$ is $\mathrm{GL}(V)$-equivariant.

Proof. - Clearly, $\pi_{!} \overline{\mathbb{Q}}_{\ell}$ is $\mathrm{GL}(V)$-equivariant. The Fourier transform preserves $\mathrm{GL}(V)$ equivariance of a perverse sheaf.

Stratify $\operatorname{ST}^{2}\left(V^{*}\right)$ by $Q_{i}(V)$, where $Q_{i}(V)$ is the locus of $b: V \rightarrow V^{*}$ such that $\operatorname{dim} \operatorname{Ker} b=i$. For $b \in \mathrm{ST}^{2}\left(V^{*}\right)$ denote by $\beta_{b}: V \rightarrow \mathbb{A}^{1}$ the map $v \mapsto\langle v, b v\rangle$. We have a usual ambiguity in identifying $\mathrm{ST}^{2}\left(V^{*}\right)$ with $\operatorname{Sym}^{2}\left(V^{*}\right): b$ goes to $\beta_{b}$ or $\frac{1}{2} \beta_{b}$. Since $S_{\psi}$ is $\operatorname{GL}(V)$-equivariant, we can view it as a perverse sheaf on $\operatorname{Sym}^{2}\left(V^{*}\right)$ unambiguously.

Lemma 3. - For $b \in Q_{0}(V)$ the complex $\mathrm{R} \Gamma_{c}\left(V, \beta_{b}^{*} \mathcal{L}_{\psi}\right)$ is a 1-dimensional vector space placed in degree $d$.

Proof. - In some basis $\beta_{b}$ is given by $\left(x_{1}, \ldots, x_{d}\right) \mapsto x_{1}^{2}+\cdots+x_{d}^{2}$. Thus we may assume $d=1$. Consider the map $\pi: \mathbb{A}^{1} \rightarrow \mathbb{A}^{1}$ given by $\pi(x)=x^{2}$. As above $\pi ! \overline{\mathbb{Q}}_{\ell} \widetilde{\sim} \mathcal{L}_{0} \oplus \mathcal{L}_{1}$ with $\mathcal{L}_{0}=\overline{\mathbb{Q}}_{\ell}$. We get $\mathrm{R} \Gamma_{c}\left(\mathbb{A}^{1}, \pi^{*} \mathcal{L}_{\psi}\right) \widetilde{\longrightarrow} \Gamma_{c}\left(\mathbb{G}_{m}, \mathcal{L} \otimes \mathcal{L}_{\psi}\right)$. The latter is a vector space of dimension one placed in degree one (a gamma-function on $\mathbb{G}_{m}$ ).

Let $\operatorname{Cov}\left(Q_{0}(V)\right) \rightarrow Q_{0}(V)$ denote the two-sheeted covering of $Q_{0}(V)$ whose fibre over $b: V \widetilde{\longrightarrow} V^{*}$ is the set of trivializations $\operatorname{det} V \widetilde{\rightarrow} k$ whose square is the one induced by $b$.

The group $\operatorname{GL}(V)$ acts transitively on $Q_{0}(V)$, so given $b \in Q_{0}(V)$ one gets an identification

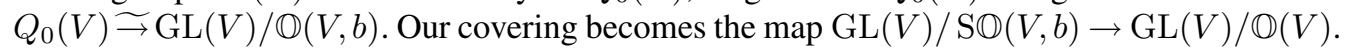

More generally, $\operatorname{GL}(V)$ acts transitively on $Q_{i}(V)$. For $b \in Q_{i}(V)$ with $\operatorname{Ker} b=V_{0}$, we can consider $b$ as an element of $\operatorname{Sym}^{2}\left(V / V_{0}\right)^{*}$. We get a parabolic $P_{0} \subset \mathrm{GL}(V)$ of automorphisms of $V$ that preserve $V_{0}$. Let $\operatorname{St}_{\mathrm{V}_{0}}$ be the preimage of $\mathbb{O}\left(V / V_{0}, b\right)$ under $P_{0} \rightarrow \mathrm{GL}\left(V / V_{0}\right)$. Then $\mathrm{St}_{\mathrm{V}_{0}}$ is the stabilizer of $b \in Q_{i}(V)$ in $\operatorname{GL}(V)$. Since $\mathrm{SO}(V, b)$ is connected, for $i<d$ there is exactly one (up to isomorphism) nonconstant $\mathrm{GL}(V)$-equivariant local system of rank one on $Q_{i}(V)$. It corresponds to the $S_{2}$-covering $\operatorname{Cov}\left(Q_{i}(V)\right) \rightarrow Q_{i}(V)$ whose fibre over $b$ is the set of trivializations $\operatorname{det}\left(V / V_{0}\right) \widetilde{\rightarrow} k$ compatible with $b$.

PROPOSITION 1.-

(1) The *-restriction of $S_{\psi}$ to $Q_{i}(V)$ is a $\mathrm{GL}(V)$-equivariant local system of rank one placed in degree $i-\frac{1}{2} d(d+1)$. For $i<d$ this local system is nonconstant and comes from the covering $\operatorname{Cov}\left(Q_{i}(V)\right) \rightarrow Q_{i}(V)$.

(2) $S_{\psi}=S_{\psi, g} \oplus S_{\psi, s}$ is a direct sum of two irreducible perverse sheaves. Here $S_{\psi, g}$ is the Goresky-MacPherson extension of $\left.S_{\psi}\right|_{Q_{0}(V)}$, and $S_{\psi, s}$ is the Goresky-MacPherson extension of $\left.S_{\psi}\right|_{Q_{1}(V)}$ under $Q_{1}(V) \hookrightarrow Q_{\geqslant 1}(V)$.

(3) We have $\mathbb{D} S_{\psi, g} \stackrel{Q}{\rightarrow} S_{\psi^{-1}, g}$ and $\mathbb{D} S_{\psi, s} \stackrel{\longrightarrow}{\rightarrow} S_{\psi^{-1}, s}$ canonically.

(4) If $V=V_{1} \oplus V_{2}$ is a direct sum of two vector spaces of dimensions $d_{1}$ and $d_{2}$ then the *-restriction of $S_{\psi} \otimes \overline{\mathbb{Q}}_{\ell}[1]\left(\frac{1}{2}\right)^{\otimes-\frac{1}{2} d(d+1)}$ to the subspace $\operatorname{Sym}^{2}\left(V_{1}^{*}\right) \oplus \operatorname{Sym}^{2}\left(V_{2}^{*}\right)$ is canonically

$$
\left(S_{\psi} \otimes S_{\psi}\right) \otimes \overline{\mathbb{Q}}_{\ell}[1]\left(\frac{1}{2}\right)^{\otimes-\frac{1}{2} d_{1}\left(d_{1}+1\right)-\frac{1}{2} d_{2}\left(d_{2}+1\right)} .
$$

Proof. - (2) A point of $Q_{i}(V)$ is given by a subspace $V_{0} \subset V$ of dimension $i$ together with non-degenerate form $b: V / V_{0} \rightarrow\left(V / V_{0}\right)^{*}$ such that $b^{*}=b$. It follows that

$$
\operatorname{dim} Q_{i}(V)=\frac{1}{2}(d-i)(d+1-i)+(d-i) i=\frac{1}{2}(d-i)(d+1+i) .
$$


From Lemma 3 applied to $V / V_{0}$ we deduce that $\left.S_{\psi}\right|_{Q_{i}(V)}$ is a local system of rank one placed in degree $i-\frac{1}{2} d(d+1)$. From (12) we see that $\mathbb{D} S_{\psi} \stackrel{\longrightarrow}{\rightarrow} S_{\psi^{-1}}$. For $0 \leqslant i \leqslant d$ we have

$$
\operatorname{dim} Q_{i}(V)=\frac{1}{2}(d-i)(d+1+i) \leqslant \frac{1}{2} d(d+1)-i,
$$

the equality holds only for $i=0$ and $i=1$. So, $S_{\psi}$ is the Goresky-MacPherson extension from the open subscheme $Q_{\leqslant 1}(V)$.

Let $S_{\psi, g}$ be the intermediate extension of $\left.S_{\psi}\right|_{Q_{0}(V)}$ to $\operatorname{Sym}^{2} V^{*}$. The $*$-restriction $\left.S_{\psi, g}\right|_{Q_{1}(V)}$ vanishes. Indeed, it should be placed in strictly negative perverse degrees, but $\left.S_{\psi}\right|_{Q_{1}(V)}$ is a perverse sheaf. Part (2) follows.

(3) Follows from (12).

(4) The composition $V_{1} \oplus V_{2} \underset{\rightarrow}{\hookrightarrow} \stackrel{\pi}{\rightarrow} \mathrm{Sym}^{2} V \stackrel{a}{\rightarrow} \mathrm{Sym}^{2} V_{1} \times \mathrm{Sym}^{2} V_{2}$ equals $\pi \times \pi$. So, $a_{!} \pi \overline{\mathbb{Q}}_{\ell} \widetilde{\rightrightarrows}\left(\pi_{!} \overline{\mathbb{Q}}_{\ell} \otimes \pi_{!} \overline{\mathbb{Q}}_{\ell}\right)$. Fourier transform interchanges $a_{!}$and the $*$-restriction under the transpose map $a^{*}: \operatorname{Sym}^{2} V_{1}^{*} \times \operatorname{Sym}^{2} V_{2}^{*} \rightarrow \operatorname{Sym}^{2} V^{*}$.

(1) Since $\left.S_{\psi}\right|_{Q_{i}(V)}$ is GL( $\left.V\right)$-equivariant, it remains to show it is nonconstant for $i<d$.

Step 1 . Start with $d=1$ case, so $Q_{0}(V) \stackrel{\hookrightarrow}{\rightarrow} \mathbb{G}_{m}$. To show that $S_{\psi}$ is nonconstant on $Q_{0}(V)$ in this case, it suffices to prove that $\mathrm{R} \Gamma_{c}\left(\mathbb{G}_{m}, S_{\psi}\right)=0$.

We will show that $\mathrm{R} \Gamma_{c}\left(\mathbb{A}^{1} \times \mathbb{G}_{m}, \beta^{*} \mathcal{L}_{\psi}\right)=0$, where the map $\beta: \mathbb{A}^{1} \times \mathbb{G}_{m} \rightarrow \mathbb{A}^{1}$ sends $(v, b)$ to $b v^{2}$. Let $\tilde{\beta}: \mathbb{A}^{1} \times \mathbb{G}_{m} \rightarrow \mathbb{A}^{1}$ be the map that sends $(v, b)$ to $b v$. For the projection $\operatorname{pr}_{1}: \mathbb{A}^{1} \times \mathbb{G}_{m} \rightarrow \mathbb{A}^{1}$ we have

$$
\operatorname{pr}_{1 !} \tilde{\beta}^{*} \mathcal{L}_{\psi} \widetilde{\rightrightarrows} j_{*} \overline{\mathbb{Q}}_{\ell}[-1]
$$

where $j: \mathbb{G}_{m} \rightarrow \mathbb{A}^{1}$ is the open immersion [16, Lemma 2.3]. Let $\pi: \mathbb{A}^{1} \rightarrow \mathbb{A}^{1}$ send $v$ to $v^{2}$. From the diagram

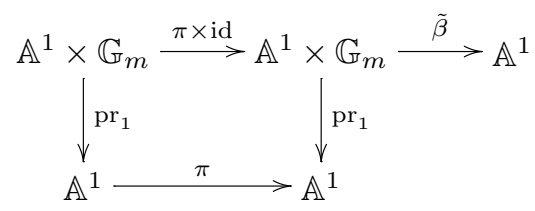

we learn that

$$
\operatorname{pr}_{1 !} \beta^{*} \mathcal{L}_{\psi} \underset{\rightarrow}{\Im} \pi^{*} \operatorname{pr}_{1 !} \tilde{\beta}^{*} \mathcal{L}_{\psi}
$$

It suffices to show that $\mathrm{R} \Gamma_{c}\left(\mathbb{A}^{1}, \pi^{*} j_{*} \overline{\mathbb{Q}}_{\ell}\right)=0$. Recall that $\pi_{!} \overline{\mathbb{Q}}_{\ell} \widetilde{\rightarrow} \overline{\mathbb{Q}}_{\ell} \oplus \mathcal{L}_{1}$, where $\mathcal{L}_{1}$ is the local system on $\mathbb{G}_{m}$ extended by zero to $\mathbb{A}^{1}$, which corresponds to the Galois covering $\pi: \mathbb{G}_{m} \rightarrow \mathbb{G}_{m}$. We get

$$
\mathrm{R} \Gamma_{c}\left(\mathbb{A}^{1}, \pi^{*} j_{*} \overline{\mathbb{Q}}_{\ell}\right) \stackrel{\mathrm{R}}{\rightarrow} \Gamma_{c}\left(\mathbb{A}^{1}, \pi_{!} \overline{\mathbb{Q}}_{\ell} \otimes j_{*} \overline{\mathbb{Q}}_{\ell}\right)=0,
$$

because $\mathrm{R} \Gamma_{c}\left(\mathbb{G}_{m}, \mathcal{L}_{1}\right)=0$ and $\mathrm{R} \Gamma_{c}\left(\mathbb{A}^{1}, j_{*} \overline{\mathbb{Q}}_{\ell}\right)=0$.

Step 2. For any $d$ and $i<d$ choose a decomposition of $V$ as a direct sum $V=W \oplus V_{1} \oplus$ $\cdots \oplus V_{d-i}$, where $\operatorname{dim} V_{j}=1$ and $\operatorname{dim} W=i$. Then $Q_{0}\left(V_{1}\right) \times \cdots \times Q_{0}\left(V_{d-i}\right) \subset Q_{i}(V)$. The restriction of $S_{\psi}$ to $Q_{0}\left(V_{1}\right) \times \cdots \times Q_{0}\left(V_{d-i}\right)$ is nonconstant by step 1 combined with (4).

Proposition 2. - A choice of a square root $i=\sqrt{-1} \in k$ yields for any $j$ an isomorphism

$$
\left.S_{\psi} \otimes S_{\psi}\right|_{Q_{j}(V)} \underset{\rightarrow}{\rightarrow} \overline{\mathbb{Q}}_{\ell}[1]\left(\frac{1}{2}\right)^{\otimes-2 j+d(d+1)} .
$$

$4^{\text {e }}$ SÉRIE - TOME $39-2006-\mathrm{N}^{\circ} 3$ 
Proof. - Let $\beta_{2}: V \times V \times \operatorname{Sym}^{2} V^{*} \rightarrow \mathbb{A}^{1}$ be the map sending $(v, u, b)$ to $\langle v, b v\rangle+\langle u, b u\rangle$. Let $p_{3}: V \times V \times \mathrm{Sym}^{2} V^{*} \rightarrow \mathrm{Sym}^{2} V^{*}$ be the projection. One checks that

$$
S_{\psi} \otimes S_{\psi} \underset{\rightarrow}{\rightarrow} p_{3 !} \beta_{2}^{*} \mathcal{L}_{\psi} \otimes \overline{\mathbb{Q}}_{\ell}[1]\left(\frac{1}{2}\right)^{\otimes 2 d+d(d+1)} .
$$

The change of variables

$$
\left\{\begin{array}{l}
x=v+i u \\
y=v-i u
\end{array}\right.
$$

makes $\beta_{2}$ to be the map sending $(x, y, b)$ to $\langle x, b y\rangle$. Summate first over $x$ with $y$ fixed, the assertion follows.

PROposition 3. - The *-restriction $\left.\operatorname{Four}_{\psi}\left(\mathcal{L}_{i}\right)\right|_{Q_{j}(V)}$ vanishes if and only if $j \neq i+$ $d \bmod 2$. In other words, if $i=d \bmod 2$ then $\operatorname{Four}_{\psi}\left(\mathcal{L}_{i}\right)$ has nontrivial fibres at $\bigcup_{j}$ even $Q_{j}(V)$. If $i \neq d \bmod 2$ then $\operatorname{Four}_{\psi}\left(\mathcal{L}_{i}\right)$ has nontrivial fibres at $\bigcup_{j \text { odd }} Q_{j}(V)$.

In particular, $\operatorname{Four}_{\psi}\left(\mathcal{L}_{i}\right)[d]\left(\frac{d}{2}\right)=S_{\psi, g}$ for $i=d \bmod 2$ and $\operatorname{Four}_{\psi}\left(\mathcal{L}_{i}\right)[d]\left(\frac{d}{2}\right)=S_{\psi, s}$ for $i \neq d \bmod 2$.

Proof. - For $d=1$ it is clear. Assume it is true for $d-1$.

The complex $\operatorname{Four}_{\psi}\left(\mathcal{L}_{j}\right)$ is $\mathrm{GL}(V)$-equivariant, and $\mathrm{GL}(V)$ acts transitively on $Q_{i}(V)$. So, for each $i$ exactly one of two sheaves $\left.\operatorname{Four}_{\psi}\left(\mathcal{L}_{0}\right)\right|_{Q_{i}(V)}$ or $\left.\operatorname{Four}_{\psi}\left(\mathcal{L}_{1}\right)\right|_{Q_{i}(V)}$ vanishes, and the other is a rank one (shifted) local system.

Write $V=V_{1} \oplus V_{2}$, where $\operatorname{dim} V_{1}=d-1$ and $\operatorname{dim} V_{2}=1$. Consider the natural map $s: \operatorname{Sym}^{2} V \rightarrow \operatorname{Sym}^{2} V_{1} \times \operatorname{Sym}^{2} V_{2}$. We have

$$
s_{!}\left(\mathcal{L}_{0}\right) \cong\left(\mathcal{L}_{0} \otimes \mathcal{L}_{0}\right) \oplus\left(\mathcal{L}_{1} \otimes \mathcal{L}_{1}\right)
$$

and

$$
s_{!}\left(\mathcal{L}_{1}\right) \widetilde{\rightrightarrows}\left(\mathcal{L}_{0} \otimes \mathcal{L}_{1}\right) \oplus\left(\mathcal{L}_{1} \otimes \mathcal{L}_{0}\right),
$$

where on the right-hand side $\mathcal{L}_{i}$ are those for $V_{1}$ and $V_{2}$.

Clearly, $Q_{i-1}\left(V_{1}\right) \times Q_{1}\left(V_{2}\right) \hookrightarrow Q_{i}(V)$ and $Q_{i}\left(V_{1}\right) \times Q_{0}\left(V_{2}\right) \hookrightarrow Q_{i}(V)$. Consider

$$
\begin{aligned}
\left.\operatorname{Four}_{\psi}\left(\mathcal{L}_{0}\right)\right|_{Q_{i}\left(V_{1}\right) \times Q_{0}\left(V_{2}\right)} \underset{\rightarrow}{ } & h^{*}\left(\operatorname{Four}_{\psi}\left(\mathcal{L}_{0}\right) \otimes \operatorname{Four}_{\psi}\left(\mathcal{L}_{0}\right)\right) \\
& \oplus h^{*}\left(\operatorname{Four}_{\psi}\left(\mathcal{L}_{1}\right) \otimes \operatorname{Four}_{\psi}\left(\mathcal{L}_{1}\right)\right),
\end{aligned}
$$

where $h: Q_{i}\left(V_{1}\right) \times Q_{0}\left(V_{2}\right) \hookrightarrow \operatorname{Sym}^{2} V_{1}^{*} \times \operatorname{Sym}^{2} V_{2}^{*}$. This isomorphism holds up to a shift and a twist.

If $i=d \bmod 2$ then $h^{*}\left(\operatorname{Four}_{\psi}\left(\mathcal{L}_{1}\right) \otimes \operatorname{Four}_{\psi}\left(\mathcal{L}_{1}\right)\right)$ is nonzero by induction hypothesis, so the LHS of (13) does not vanish, hence $\left.\operatorname{Four}_{\psi}\left(\mathcal{L}_{0}\right)\right|_{Q_{i}(V)}$ does not vanish either.

If $i \neq d \bmod 2$ then the RHS of (13) vanishes by induction hypothesis, so the LHS also vanishes. Thus, $\left.\operatorname{Four}_{\psi}\left(\mathcal{L}_{0}\right)\right|_{Q_{i}(V)}$ vanishes.

4.2. Assume $d \geqslant 1$. Let $Y(V)$ be the moduli scheme of pairs: a one-dimensional subspace $V_{0} \subset V$ and $b \in \operatorname{Sym}^{2}\left(V / V_{0}\right)^{*}$. The projection $Y(V) \rightarrow \operatorname{Gr}(1, V)$ is a vector bundle, where $\operatorname{Gr}(1, V)$ denotes the Grassmanian of one-dimensional subspaces in $V$. Let $\alpha: Y(V) \rightarrow \operatorname{Sym}^{2} V^{*}$ be the map sending the above point to the composition

$$
V \rightarrow V / V_{0} \stackrel{b}{\longrightarrow}\left(V / V_{0}\right)^{*} \hookrightarrow V^{*}
$$


Clearly, $\alpha$ factors through $Q_{\geqslant 1}(V) \hookrightarrow \operatorname{Sym}^{2} V^{*}$. Note that $Y(V)$ is smooth.

PROPOSITION 4. - The map $\alpha: Y(V) \rightarrow Q_{\geqslant 1}(V)$ is proper surjective and semi-small.

Proof. - Stratify $Q_{\geqslant 1}(V)$ by $Q_{i}(V)$ for $i \geqslant 1$. The fibre of $\alpha$ over a point $b \in Q_{i}(V)$ is the projective space of 1 -subspaces in $V^{\prime}$, where $V^{\prime}$ is the kernel of $b$. So, $\operatorname{dim} \alpha^{-1}(b)=i-1$ and $\operatorname{dim} Q_{i}(V)=\frac{1}{2}(d-i)(d+1+i)$. We get

$$
2 \operatorname{dim} \alpha^{-1}(b) \leqslant \operatorname{codim}_{Q \geqslant 1}(V) Q_{i}(V),
$$

the equality holds only for $i=1,2$.

\subsection{Relative version}

Let now $S$ be a smooth scheme, $V \rightarrow S$ be a vector bundle of rank $d$. Define $S_{\psi} \in$ $\mathrm{D}\left(\mathrm{Sym}^{2} V^{*}\right)$ by (12), so $S_{\psi}$ is a shifted perverse sheaf.

As above, $\operatorname{Sym}^{2} V^{*}$ is stratified by locally closed subschemes $Q_{i}(V)$, they are equipped with morphisms $Q_{i}(V) \rightarrow \operatorname{Gr}(i, V)$ over $S$.

We also have the $S_{2}$-coverings $\operatorname{Cov}\left(Q_{i}(V)\right) \rightarrow Q_{i}(V)$. For an $S$-scheme $S^{\prime}$, the $S^{\prime}$-points of $\operatorname{Cov}\left(Q_{i}(V)\right)$ are collections: a rank $i$ subbundle $\left.V_{0} \subset V\right|_{S^{\prime}}$, an isomorphism $b: V / V_{0} \rightarrow\left(V / V_{0}\right)^{*}$ of $\mathcal{O}_{S^{\prime}}$-modules with $b^{*}=b$, and a compatible trivialization $\operatorname{det}\left(V / V_{0}\right) \widetilde{\rightarrow} \mathcal{O}_{S^{\prime}}$.

Propositions 1, 2 and 3 hold in relative situation (one only changes a shift and a twist in (3) of Proposition 1).

\subsection{Finite-dimensional theta-sheaf}

This subsection is not used in the proofs and may be skipped.

Let $M$ be a symplectic $k$-vector space of dimension $2 d$. Write $\mathcal{L}(M)$ for the scheme of Lagrangian subspaces of $M$. Set $Y=\mathcal{L}(M) \times \mathcal{L}(M)$. Consider the line bundle $\mathcal{A}$ on $Y$ with fibre $\left(\operatorname{det} L_{1}\right) \otimes\left(\operatorname{det} L_{2}\right)$ over $\left(L_{1}, L_{2}\right) \in Y$. We view it as $\mathbb{Z} / 2 \mathbb{Z}$-graded purely of degree zero.

Let $\widetilde{Y}$ denote the stack of square roots of $\mathcal{A}$. The $\mu_{2}$-gerbe $\widetilde{Y} \rightarrow Y$ is nontrivial. The group $\operatorname{Sp}(M)$ acts naturally on $Y$, and $\mathcal{A}$ is $\operatorname{Sp}(M)$-equivariant, so $\operatorname{Sp}(M)$ acts also on $\widetilde{Y}$.

We are going to construct a $\operatorname{Sp}(M)$-equivariant perverse sheaf $S_{M}$ on $\widetilde{Y}$ such that $-1 \in \mu_{2}$ acts on $S_{M}$ as -1 .

The $\operatorname{Sp}(M)$-orbits on $Y$ are indexed by $i=0, \ldots, d$. The orbit $Y_{i}$ is given by $\operatorname{dim}\left(L_{1} \cap L_{2}\right)=i$.

LEMMA 4. - The restriction of $\mathcal{A}$ to each $Y_{i}$ admits a canonical $\mathbb{S p}(M)$-equivariant square root.

Proof. - For $L_{1}, L_{2} \in \mathcal{L}(M)$ let $\left(L_{1} \cap L_{2}\right)^{\perp} \subset M$ denotes the orthogonal complement to $L_{1} \cap L_{2}$. The symplectic form on $\left(L_{1} \cap L_{2}\right)^{\perp} /\left(L_{1} \cap L_{2}\right)$ induces an isomorphism $L_{2} /\left(L_{1} \cap\right.$ $\left.L_{2}\right) \stackrel{\rightrightarrows}{\rightarrow}\left(L_{1} / L_{1} \cap L_{2}\right)^{*}$. This yields a $\mathbb{Z} / 2 \mathbb{Z}$-graded isomorphism $\left(\operatorname{det} L_{1}\right) \otimes\left(\operatorname{det} L_{2}\right) \widetilde{\hookrightarrow}$ $\operatorname{det}\left(L_{1} \cap L_{2}\right)^{\otimes 2}$. By 3.1.2, we are done.

Let $W$ denote the nontrivial local system of rank one on $B\left(\mu_{2}\right)$ corresponding to the covering Spec $k \rightarrow B\left(\mu_{2}\right)$. Let $\widetilde{Y}_{i}$ denote the restriction of the gerbe $\tilde{Y} \rightarrow Y$ to $Y_{i}$, so $\widetilde{Y}_{i} \widetilde{\rightarrow} Y_{i} \times B\left(\mu_{2}\right)$ canonically.

Definition 2. - Let $S_{M, g}$ (respectively, $S_{M, s}$ ) denote the Goresky-MacPherson extension of

$$
\left(\overline{\mathbb{Q}}_{\ell} \otimes W\right)[\operatorname{dim} Y]\left(\frac{\operatorname{dim} Y}{2}\right)
$$

$4^{\text {e }}$ SÉRIE - TOME $39-2006-\mathrm{N}^{\circ} 3$ 
from $\tilde{Y}_{0}$ to $\tilde{Y}$ (respectively, of $\left(\overline{\mathbb{Q}}_{\ell} \otimes W\right)[\operatorname{dim} Y-1]\left(\frac{\operatorname{dim} Y-1}{2}\right)$ from $\widetilde{Y}_{1}$ to $\left.\tilde{Y}\right)$. Set $S_{M}=S_{M, g} \oplus S_{M, s}$.

Denote by $\mathcal{Y}$ the stack quotient $Y / \mathbb{S p}(M)$. Write $\widetilde{\mathcal{Y}} \rightarrow \mathcal{Y}$ for the corresponding gerbe of square roots of $\mathcal{A}$. We may view $S_{M}$ as a perverse sheaf on $\widetilde{\mathcal{Y}}$.

Fix a Lagrangian subspace $V \subset M$, let $P_{V} \subset \mathbb{S p}(M)$ be the Seigel parabolic subgroup preserving $V$. We have canonical isomorphisms of stacks

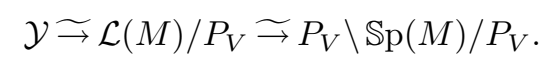

One may view $\mathcal{A}$ as a line bundle on $\mathcal{L}(M) / P_{V}$ with fibre $(\operatorname{det} V) \otimes(\operatorname{det} L)$.

Fix a splitting $V^{*} \rightarrow M$ of $0 \rightarrow V \rightarrow M \rightarrow V^{*} \rightarrow 0$. Denote by $P_{V}^{-} \subset \operatorname{Sp}(M)$ the Seigel parabolic subgroup preserving $V^{*} \subset M$. Let $Z \subset \mathcal{L}(M)$ be the open $P_{V}^{-}$-orbit, that is

$$
Z=\left\{L \in \mathcal{L}(M) \mid L \cap V^{*}=0\right\} .
$$

The map $\operatorname{Sym}^{2} V^{*} \rightarrow Z$ sending $b: V \rightarrow V^{*}$ to $L=\{v+b v \in M \mid v \in V\}$ is an isomorphism commuting with the action of $\mathrm{GL}(V)$. Denote by $\mathcal{Z}$ the stack quotient $Z / \mathrm{GL}(V)$. View $S_{\psi}$ introduced in Section 4.1 as a perverse sheaf on $\mathcal{Z}$.

Denote by $\nu$ the composition (of an open immersion followed by a smooth map)

$$
\mathcal{Z} \hookrightarrow \mathcal{L}(M) / \mathrm{GL}(V) \rightarrow \mathcal{L}(M) / P_{V}=\mathcal{Y}
$$

The map $\nu: \mathcal{Z} \rightarrow \mathcal{Y}$ is smooth, surjective and representable. It factors naturally as $\mathcal{Z} \stackrel{\tilde{\nu}}{\longrightarrow} \widetilde{\mathcal{Y}} \rightarrow \mathcal{Y}$.

PROPOSITION 5. - There are isomorphisms of perverse sheaves on $\mathcal{Z}$

$$
\tilde{\nu}^{*} S_{M, g} \otimes \overline{\mathbb{Q}}_{\ell}[1]\left(\frac{1}{2}\right)^{\otimes \operatorname{dim} \mathcal{Z}-\operatorname{dim} \mathcal{Y}} \stackrel{\longrightarrow}{\longrightarrow} S_{\psi, g}
$$

and

$$
\tilde{\nu}^{*} S_{M, s} \otimes \overline{\mathbb{Q}}_{\ell}[1]\left(\frac{1}{2}\right)^{\otimes \operatorname{dim} \mathcal{Z}-\operatorname{dim} \mathcal{Y}} \stackrel{\longrightarrow}{\rightarrow} S_{\psi, s} .
$$

(Once $i=\sqrt{-1} \in k$ is fixed, such isomorphisms are well defined up to multiplication by \pm 1 .)

Proof. - The stack $\mathcal{Z}$ is stratified by $\mathcal{Z}_{i}=Q_{i}(V) / \mathrm{GL}(V)$, the quotient being taken in stack sense. Let $\mathcal{Y}_{i}$ denote the stack quotient $Y_{i} / \operatorname{Sp}(M)$. Note that $\mathcal{Z}_{i}$ identifies with $\mathcal{Z} \times \mathcal{Y} \mathcal{Y}_{i}$ for $i=0, \ldots, d$.

Let $\ddot{\mathcal{Y}}_{i}$ denote the restriction of the gerbe $\widetilde{\mathcal{Y}}$ to $\mathcal{Y}_{i}$, so $\widetilde{\mathcal{Y}}_{i} \widetilde{\rightrightarrows} \mathcal{Y}_{i} \times B\left(\mu_{2}\right)$ canonically. Remind the covering $\operatorname{Cov}\left(Q_{i}(V)\right) \rightarrow Q_{i}(V)$ from Section 4.1. It is $\operatorname{GL}(V)$-equivariant, so the stack quotient $\operatorname{Cov}\left(\mathcal{Z}_{i}\right)=\operatorname{Cov}\left(Q_{i}(V)\right) / \operatorname{GL}(V)$ is a two-sheeted covering of $\mathcal{Z}_{i}$. For each $i$ we have a Cartesian square

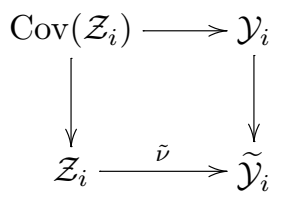

Our assertion follows now from Proposition 1. 
Remark 3. - Write ${ }_{M} Y$ (respectively, ${ }_{M} \widetilde{\mathcal{Y}}$ ) to express the dependence on $M$. If $M, M^{\prime}$ are two symplectic spaces over $k$ of dimensions $d, d^{\prime}$, consider the map $\tau_{M, M^{\prime}}:{ }_{M} Y \times{ }_{M^{\prime}} Y \rightarrow{ }_{M \oplus M^{\prime}} Y$ sending $\left(L_{1}, L_{2}\right),\left(L_{1}^{\prime}, L_{2}^{\prime}\right)$ to $\left(L_{1} \oplus L_{1}^{\prime}, L_{2} \oplus L_{2}^{\prime}\right)$. It yields a map

$$
\tilde{\tau}_{M, M^{\prime}}:{ }_{M} \tilde{\mathcal{Y}} \times{ }_{M^{\prime}} \tilde{\mathcal{Y}} \rightarrow{ }_{M \oplus M^{\prime}} \tilde{\mathcal{Y}} .
$$

From (4) of Proposition 1 it follows that $\tilde{\tau}_{M, M^{\prime}}^{*} S_{M \oplus M^{\prime}} \underset{\rightarrow}{\hookrightarrow} S_{M} \otimes S_{M^{\prime}}\left[2 d d^{\prime}\right]\left(d d^{\prime}\right)$ canonically.

\section{Fourier coefficients of Aut for Siegel parabolic}

5.1. Write $\operatorname{Bun}_{P}=\operatorname{Bun}_{P_{n}}$. So, $\operatorname{Bun}_{P}$ classifies $L \in \operatorname{Bun}_{n}$ together with an exact sequence $0 \rightarrow \operatorname{Sym}^{2} L \rightarrow ? \rightarrow \Omega \rightarrow 0$ on $X$. It induces an exact sequence

$$
0 \rightarrow L \rightarrow M \rightarrow L^{*} \otimes \Omega \rightarrow 0 .
$$

The map $\nu_{n}: \operatorname{Bun}_{P} \rightarrow \operatorname{Bun}_{G}$ is also denoted by $\nu$.

LEMmA 5. - The map $\nu: \operatorname{Bun}_{P} \rightarrow \operatorname{Bun}_{G}$ factors as the composition $\operatorname{Bun}_{P} \stackrel{\tilde{\nu}}{\longrightarrow} \widetilde{\operatorname{Bun}}_{G} \stackrel{\mathfrak{r}}{\longrightarrow}$ $\operatorname{Bun}_{G}$.

Proof. - The sequence (14) yields a $\mathbb{Z} / 2 \mathbb{Z}$-graded isomorphism

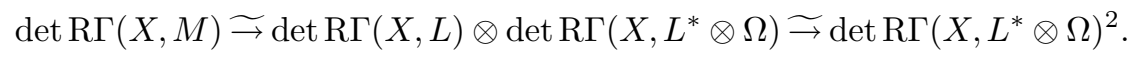

Define $\tilde{\nu}$ by letting $\mathcal{B}=\operatorname{det} \mathrm{R} \Gamma\left(X, L^{*} \otimes \Omega\right)$ together with $\mathcal{B}^{2} \widetilde{\mathcal{A}}$ given by (15). By 3.1.2, $\tilde{\nu}$ is well-defined.

Let ${ }^{0} \operatorname{Bun}_{P} \subset \operatorname{Bun}_{P}$ be the open substack given by $\mathrm{H}^{0}\left(X, \operatorname{Sym}^{2} L\right)=0$. One checks that both $\nu:{ }^{0} \operatorname{Bun}_{P} \rightarrow \operatorname{Bun}_{G}$ and $\tilde{\nu}:{ }^{0} \operatorname{Bun}_{P} \rightarrow \widetilde{\operatorname{Bun}_{G}}$ are smooth.

Lemma 6. - The map $\nu:{ }^{0} \operatorname{Bun}_{P} \rightarrow \operatorname{Bun}_{G}$ is surjective, so $\tilde{\nu}:{ }^{0} \operatorname{Bun}_{P} \rightarrow \widetilde{\operatorname{Bun}_{G}}$ is also surjective.

Proof. - Let $M$ be a $k$-point of $\operatorname{Bun}_{G}$. It admits a line subbundle $L_{1}$ with $\operatorname{deg} L_{1}<0$. Let $L_{-1} \subset M$ be the orthogonal complement to $L_{1}$, so $L_{-1} / L_{1} \in \operatorname{Bun}_{G_{n-1}}$ naturally. Continuing this procedure for $L_{-1} / L_{1}$ and so on, we get a flag of isotropic subbundles $L_{1} \subset \cdots \subset L_{n} \subset M$. Then $\left(L_{n} \subset M\right)$ is a $k$-point of ${ }^{0} \mathrm{Bun}_{P}$.

\subsection{The sheaf $S_{P, \psi}$ on $\operatorname{Bun}_{P}$}

Write $\operatorname{Bun}_{n}^{d}$ (respectively, $\operatorname{Bun}_{P}^{d}$ ) for the connected component of the corresponding stack given by $\operatorname{deg} L=d$.

Write ${ }_{c} \operatorname{Bun}_{n} \subset \operatorname{Bun}_{n}$ for the open substack given by $\mathrm{H}^{0}(X, L)=0$. Let $\mathcal{V} \rightarrow \operatorname{Bun}_{n}$ be the stack whose fibre over $L \in \operatorname{Bun}_{n}$ is $\operatorname{Hom}(L, \Omega)$. Let ${ }_{c} \mathcal{V} \rightarrow{ }_{c} \operatorname{Bun}_{n}$ be the preimage of ${ }_{c} \operatorname{Bun}_{n}$, over ${ }_{c} \operatorname{Bun}_{n}^{d}$ this is a vector bundle of rank $n(g-1)-d$.

Let $\mathcal{X}=\mathcal{V} \times{ }_{\operatorname{Bun}_{n}} \operatorname{Bun}_{P}$ and $p: \mathcal{X} \rightarrow \operatorname{Bun}_{P}$ be the projection. Let $q: \mathcal{X} \rightarrow \mathbb{A}^{1}$ be the map sending $s \in \mathrm{H}^{0}\left(X, L^{*} \otimes \Omega\right)$ to the pairing of $0 \rightarrow \operatorname{Sym}^{2} L \rightarrow ? \rightarrow \Omega \rightarrow 0$ with

$$
s \otimes s \in \mathrm{H}^{0}\left(X,\left(\operatorname{Sym}^{2} L^{*}\right) \otimes \Omega^{2}\right) .
$$

Definition 3. - Set $S_{P, \psi}=p_{!} q^{*} \mathcal{L}_{\psi} \otimes \overline{\mathbb{Q}}_{\ell}[1]\left(\frac{1}{2}\right)^{\otimes \operatorname{dim} \mathcal{X}}$, where $\operatorname{dim} \mathcal{X}$ is the dimension of the corresponding connected component of $\mathcal{X}$.

$4^{\text {e }}$ SÉRIE - TOME $39-2006-\mathrm{N}^{\circ} 3$ 
Let $\mathcal{V}_{2} \rightarrow \operatorname{Bun}_{n}$ be the stack whose fibre over $L \in \operatorname{Bun}_{n}$ is $\operatorname{Hom}\left(\operatorname{Sym}^{2} L, \Omega^{2}\right)$. Let $\pi_{2}: \mathcal{V} \rightarrow \mathcal{V}_{2}$ be the map sending $s \in \operatorname{Hom}(L, \Omega)$ to $s \otimes s$. Note that $\pi_{2}$ is finite, a $S_{2}$-covering over the image $\operatorname{Im} \pi_{2}$ with removed zero section. By definition,

$$
S_{P, \psi} \underset{\rightarrow}{\rightarrow} \operatorname{Four}_{\psi}\left(\pi_{2 !} \overline{\mathbb{Q}}_{\ell}\right) \otimes \overline{\mathbb{Q}}_{\ell}[1]\left(\frac{1}{2}\right)^{\otimes \operatorname{dim} \mathcal{V}},
$$

where $\operatorname{Four}_{\psi}: \mathrm{D}\left(\mathcal{V}_{2}\right) \rightarrow \mathrm{D}\left(\operatorname{Bun}_{P}\right)$ is the Fourier transform functor. Note that $S_{2}$ acts on $S_{P, \psi}$.

Let ${ }_{c} \operatorname{Bun}_{P}$ denote the preimage of ${ }_{c} \operatorname{Bun}_{n}$ in $\operatorname{Bun}_{P}$. We see that over each connected component of ${ }_{c} \operatorname{Bun}_{P}, S_{P, \psi}$ is a direct sum of two irreducible perverse sheaves and $\mathbb{D}\left(S_{P, \psi}\right) \widetilde{\rightarrow} S_{P, \psi^{-1}}$.

Let $\operatorname{Sym}^{2}{ }_{c} \mathcal{V} \rightarrow{ }_{c} \operatorname{Bun}_{n}$ denote the symmetric square of the vector bundle ${ }_{c} \mathcal{V} \rightarrow{ }_{c} \mathrm{Bun}_{n}$. Let $\pi:{ }_{c} \mathcal{V} \rightarrow \operatorname{Sym}^{2}{ }_{c} \mathcal{V}$ be the map sending $s \in \operatorname{Hom}(L, \Omega)$ to $s \otimes s$. Then $\pi_{2}$ decomposes as

$$
{ }_{c} \mathcal{V} \stackrel{\pi}{\longrightarrow} \operatorname{Sym}^{2}{ }_{c} \mathcal{V} \stackrel{f^{*}}{\longrightarrow}{ }_{c} \mathcal{V}_{2}
$$

Given $L \in \operatorname{Bun}_{n}$, the transpose to the linear map $\operatorname{Sym}^{2} \mathrm{H}^{0}\left(X, L^{*} \otimes \Omega\right) \rightarrow \operatorname{Hom}\left(\operatorname{Sym}^{2} L, \Omega^{2}\right)$ is

$$
\mathrm{H}^{1}\left(X,\left(\operatorname{Sym}^{2} L\right) \otimes \Omega^{-1}\right) \rightarrow \operatorname{Sym}^{2} \mathrm{H}^{1}(X, L) .
$$

It defines a morphism of stacks $f:{ }_{c} \operatorname{Bun}_{P} \rightarrow \operatorname{Sym}^{2}{ }_{c} \mathcal{V}^{*}$ over ${ }_{c} \operatorname{Bun}_{n}$.

We have the sheaf $S_{\psi}$ on $\mathrm{Sym}^{2}{ }_{c} \mathcal{V}^{*}$ defined in Section 4.3. From (16) we conclude that

$$
S_{P, \psi} \underset{\rightrightarrows}{\longrightarrow} f^{*} S_{\psi} \otimes \overline{\mathbb{Q}}_{\ell}[1]\left(\frac{1}{2}\right)^{\otimes \operatorname{dim} \mathcal{X}-r-\frac{1}{2} r(r+1)}
$$

canonically over ${ }_{c} \operatorname{Bun}_{P}$, where $r$ and $\operatorname{dim} \mathcal{X}$ are functions of the corresponding connected component with $r\left({ }_{c} \operatorname{Bun}_{P}^{d}\right)=n(g-1)-d$.

Denote by $S_{P, \psi, g}$ (respectively, by $S_{P, \psi, s}$ ) the direct summand of $S_{P, \psi}$ obtained by replacing $S_{\psi}$ by $S_{\psi, g}$ (respectively, by $S_{\psi, s}$ ) in (17). Both $S_{P, \psi, g}$ and $S_{P, \psi, s}$ are irreducible perverse sheaves over each connected component of ${ }_{c} \mathrm{Bun}_{P}$.

Note that ${ }^{0} \operatorname{Bun}_{P} \subset{ }_{c} \operatorname{Bun}_{P}$.

Remark 4. - Consider $\chi(L)$ as a function of a connected component of ${ }_{c} \mathrm{Bun}_{P}$. By Proposition 3, over a given connected component of ${ }_{c} \operatorname{Bun}_{P}$, the $S_{2}$-invariants of $S_{P, \psi}$ are $S_{P, \psi, g}$ for $\chi(L)$ even and $S_{P, \psi, s}$ for $\chi(L)$ odd.

5.3. Recall the stratification of $\operatorname{Sym}^{2}{ }_{c} \mathcal{V}^{*}$ by locally closed substacks $Q_{i}\left({ }_{c} \mathcal{V}\right)$ and the coverings $\operatorname{Cov}\left(Q_{i}\left({ }_{c} \mathcal{V}\right)\right) \rightarrow Q_{i}\left({ }_{c} \mathcal{V}\right)$ defined in Section 4.3.

Set ${ }_{i} \operatorname{Bun}_{P}=\nu^{-1}\left({ }_{i} \operatorname{Bun}_{G}\right)$ and ${ }_{i, c} \operatorname{Bun}_{P}={ }_{c} \operatorname{Bun}_{P} \cap{ }_{i} \operatorname{Bun}_{P}$. For a point of ${ }_{c} \operatorname{Bun}_{P}$ the exact sequence (14) yields an exact sequence

$$
0 \rightarrow \mathrm{H}^{0}(X, M) \rightarrow \mathrm{H}^{0}\left(X, L^{*} \otimes \Omega\right) \stackrel{b}{\longrightarrow} \mathrm{H}^{1}(X, L) \rightarrow \mathrm{H}^{1}(X, M) \rightarrow 0 .
$$

Thus, we get a commutative diagram

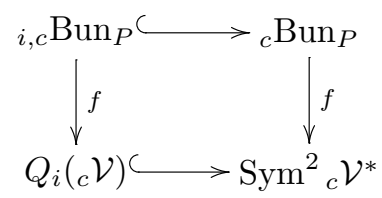


Let ${ }_{i} \rho_{P}: \operatorname{Cov}\left({ }_{i, c} \operatorname{Bun}_{P}\right) \rightarrow{ }_{i, c} \operatorname{Bun}_{P}$ be the covering obtained from $\operatorname{Cov}\left(Q_{i}\left({ }_{c} \mathcal{V}\right)\right) \rightarrow Q_{i}\left({ }_{c} \mathcal{V}\right)$ by the base change $f:{ }_{i, c} \operatorname{Bun}_{P} \rightarrow Q_{i}\left({ }_{c} \mathcal{V}\right)$.

Proposition 6. - For $i \geqslant 0$ there is a Cartesian square

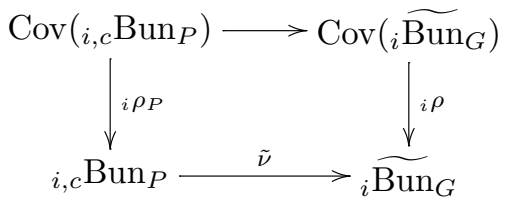

Proof. - Let $S$ be a scheme. Assume given an $S$-point of ${ }_{i, c} \operatorname{Bun}_{P}$. It yields locally free $\mathcal{O}_{S}$-modules $V_{0}=\mathrm{H}^{0}(X, M)$ and $V=\mathrm{H}^{0}\left(X, L^{*} \otimes \Omega\right)$ included into an exact sequence of $\mathcal{O}_{S}$-modules (a relative version of (18))

$$
0 \rightarrow V_{0} \rightarrow V \stackrel{b}{\longrightarrow} V^{*} \rightarrow V_{0}^{*} \rightarrow 0
$$

with $b^{*}=b$. The $\mathcal{O}_{S \times X}$-module $L$ together with the morphism of $\mathcal{O}_{S}$-modules $V \stackrel{b}{\longrightarrow} V^{*}$ defines the corresponding $S$-point of $Q_{i}\left({ }_{c} \mathcal{V}\right)$.

We have an isomorphism of $\mathcal{O}_{S}$-modules $\mathcal{B}=\operatorname{det} \mathrm{R} \Gamma\left(X, L^{*} \otimes \Omega\right) \widetilde{\longrightarrow} \operatorname{det} V$, because $\mathrm{H}^{0}(X, L)=0$. We also have an isomorphism of $\mathcal{O}_{S}$-modules $t: \mathcal{B}^{2} \widetilde{\nearrow} \operatorname{det} \mathrm{R} \Gamma(X, M) \widetilde{\rightrightarrows}$ $\left(\operatorname{det} V_{0}\right)^{2}$ given by (15).

A lifting of the corresponding $S$-point of ${ }_{i} \widetilde{\operatorname{Bun}}_{G}$ to $\operatorname{Cov}\left({ }_{i} \widetilde{\operatorname{Bun}}_{G}\right)$ is an isomorphism of $\mathcal{O}_{S}$-modules $\mathcal{B} \widetilde{\longrightarrow} \operatorname{det} V_{0}$ whose square is $t$. The corresponding category is the category of $S$-points of $\operatorname{Cov}\left({ }_{i, c} \operatorname{Bun}_{P}\right)$.

PROPOSITION 7. - There are isomorphisms of perverse sheaves on ${ }^{0} \mathrm{Bun}_{P}$

$$
\tilde{\nu}^{*} \operatorname{Aut}_{g} \otimes \overline{\mathbb{Q}}_{\ell}[1]\left(\frac{1}{2}\right)^{\otimes \operatorname{dimBun} P-d_{G}} \underset{\rightarrow}{\longrightarrow} S_{P, \psi, g}
$$

and

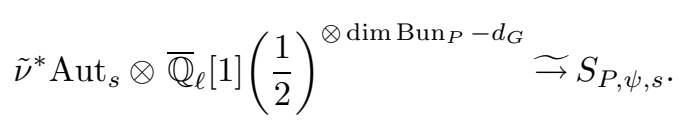

Here $\operatorname{dim} \operatorname{Bun}_{P}$ denotes the dimension of the corresponding connected component of $\operatorname{Bun}_{P}$. (Once $\sqrt{-1} \in k$ is fixed, the above isomorphisms are well-defined up to a sign.)

Proof. - Recall that $S_{P, \psi, g}$ and $S_{P, \psi, s}$ are irreducible perverse sheaves over each connected component of ${ }_{c} \operatorname{Bun}_{P}$. By relative version of Proposition 1, $S_{P, \psi, g}$ over ${ }_{0, c} \operatorname{Bun}_{P}$ (respectively, $S_{P, \psi, s}$ over ${ }_{1, c} \operatorname{Bun}_{P}$ ) is a nonconstant local system of rank one corresponding to the covering $\operatorname{Cov}\left(0,{ }_{, c} \operatorname{Bun}_{P}\right) \rightarrow{ }_{0, c} \operatorname{Bun}_{P}\left(\right.$ respectively, $\left.\operatorname{Cov}\left({ }_{1, c} \operatorname{Bun}_{P}\right) \rightarrow{ }_{1, c} \operatorname{Bun}_{P}\right)$. Moreover, for any $i$

$$
\left.\left(S_{P, \psi} \otimes S_{P, \psi}\right)\right|_{i, c} \operatorname{Bun}_{P} \underset{\rightarrow}{\rightarrow} \overline{\mathbb{Q}}_{\ell}[2](1)^{\otimes \operatorname{dim} \operatorname{Bun}_{P}-i}
$$

by Proposition 2 (this requires a choice of $\sqrt{-1} \in k$ ).

By Proposition 6, for each $i$ we get isomorphisms

$$
\left.\tilde{\nu}^{*}\left({ }_{i} \mathrm{Aut}\right)\right|_{i, c} \mathrm{Bun}_{P} \underset{\rightarrow}{\sim} \operatorname{Hom}_{S_{2}}\left(\operatorname{sign},\left({ }_{i} \rho_{P}\right) ! \overline{\mathbb{Q}}_{\ell}\right) .
$$

$4^{\text {e }}$ SÉRIE - TOME $39-2006-\mathrm{N}^{\circ} 3$ 
In particular,

$$
\left.\tilde{\nu}^{*}\left({ }_{i} \text { Aut } \otimes_{i} \text { Aut }\right)\right|_{i, c} \text { Bun } P_{P} \stackrel{\rightarrow}{\rightarrow} \overline{\mathbb{Q}}_{\ell} .
$$

Set $_{i}^{0} \operatorname{Bun}_{P}={ }^{0} \operatorname{Bun}_{P} \cap{ }_{i} \operatorname{Bun}_{P}$. By construction, $S_{P, \psi, s}$ is perverse over ${ }_{1, c} \operatorname{Bun}_{P}$, hence also over ${ }_{1}^{0} \operatorname{Bun}_{P}$. Since ${ }_{1}^{0} \operatorname{Bun}_{P} \rightarrow{ }_{1} \operatorname{Bun}_{G}$ is smooth and surjective, Propositions 1 and 6 imply that ${ }_{1}$ Aut $\otimes \overline{\mathbb{Q}}_{\ell}[1]\left(\frac{1}{2}\right)^{\otimes d_{G}-1}$ is perverse on ${ }_{1} \mathrm{Bun}_{G}$. So, Definition 1 makes sense.

For each connected component ${ }^{0} \operatorname{Bun}_{P}^{d}$ of ${ }^{0} \operatorname{Bun}_{P}$ the $\operatorname{stack}^{0} \operatorname{Bun}_{P}^{d} \cap{ }_{i} \operatorname{Bun}_{P}$ is nonempty for $i=0,1$. Since $\tilde{\nu}:{ }^{0} \operatorname{Bun}_{P} \rightarrow \widetilde{\operatorname{Bun}}_{G}$ is smooth, our assertion follows.

Proof of Theorem 1. - For each $d$ the map $\tilde{\nu}:{ }^{0} \operatorname{Bun}_{P}^{d} \rightarrow \widetilde{\operatorname{Bun}_{G}}$ is smooth with connected fibres, and $\tilde{\nu}:{ }^{0} \operatorname{Bun}_{P} \rightarrow \widetilde{\operatorname{Bun}}_{G}$ is surjective. So, by Proposition 7 it suffices to construct isomorphisms

$$
\left.S_{P, \psi}\right|_{i} \mathrm{Bun}_{P} \underset{\rightarrow}{\longrightarrow} \tilde{\nu}^{*}\left({ }_{i} \mathrm{Aut}\right) \otimes \overline{\mathbb{Q}}_{\ell}[1]\left(\frac{1}{2}\right)^{\otimes \operatorname{dim} \operatorname{Bun}_{P}-i}
$$

over $_{i}^{0} \operatorname{Bun}_{P}$. We have them by Proposition 6 combined with relative version of Proposition 1 . Proposition 3 implies the second part of the theorem.

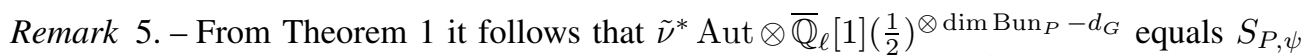
in the Grothendieck group $K\left({ }_{c} \operatorname{Bun}_{P}\right)$ over ${ }_{c} \mathrm{Bun}_{P}$, which is bigger than ${ }^{0} \mathrm{Bun}_{P}$. We expect that actually the isomorphisms of Proposition 7 hold over ${ }_{c} \operatorname{Bun}_{P}$.

\section{Constant terms of Aut for maximal parabolics}

6.1. Recall the smooth map $\eta_{k}: \operatorname{Bun}_{P_{k}} \rightarrow \operatorname{Bun}_{Q_{k}}$ (cf. Section 3.3). Under each of the two projections $\operatorname{Bun}_{P_{k}} \times_{\operatorname{Bun}_{Q_{k}}} \operatorname{Bun}_{P_{k}} \rightarrow \operatorname{Bun}_{P_{k}}$ the stack $\operatorname{Bun}_{P_{k}} \times_{\operatorname{Bun}_{Q_{k}}} \operatorname{Bun}_{P_{k}}$ identifies with the one classifying $\left(L_{1} \subset L_{-1} \subset M\right) \in \operatorname{Bun}_{P_{k}}$ together with an exact sequence $0 \rightarrow \operatorname{Sym}^{2} L_{1} \rightarrow$ $? \rightarrow \Omega \rightarrow 0$, the projection being the forgetful map.

Let $\nu_{k, n}: \operatorname{Bun}_{P_{k, n}} \rightarrow \operatorname{Bun}_{P}$ be the stack classifying $\left(0 \rightarrow \operatorname{Sym}^{2} L \rightarrow ? \rightarrow \Omega \rightarrow 0\right) \in \operatorname{Bun}_{P}$ together with a subbundle $L_{1} \subset L$ with $L_{1} \in \operatorname{Bun}_{k}$.

LEMMA 7. - The map $\eta_{k}: \operatorname{Bun}_{P_{k}} \rightarrow \operatorname{Bun}_{Q_{k}}$ is surjective.

Proof. - Consider a $k$-point of $\operatorname{Bun}_{Q_{k}}$ given by a flag $\left(L_{1} \subset L_{-1}\right)$ of vector bundles on $X$ with $L_{-1} / L_{1} \in \operatorname{Bun}_{G_{n-k}}$. Let show that the fibre of $\eta_{k}$ over it is nonempty.

Pick a Lagrangian subbundle $\mathcal{B} \subset L_{-1} / L_{1}$ such that $\mathrm{H}^{1}\left(X, \mathcal{B}^{*} \otimes L_{1}\right)=0$, it always exists. Let $L \subset L_{-1}$ be the preimage of $\mathcal{B}$ under $L_{-1} \rightarrow L_{-1} / L_{1}$. The exact sequence $0 \rightarrow L_{1} \rightarrow L \rightarrow$ $\mathcal{B} \rightarrow 0$ splits, we fix a splitting $L \widetilde{\neg} L_{1} \oplus \mathcal{B}$. Then our $k$-point of $\operatorname{Bun}_{Q_{k}}$ becomes the data of two exact sequences

$$
0 \rightarrow \operatorname{Sym}^{2} \mathcal{B} \rightarrow ? \rightarrow \Omega \rightarrow 0
$$

and

$$
0 \rightarrow L_{1} \rightarrow ? \rightarrow \mathcal{B}^{*} \otimes \Omega \rightarrow 0
$$

Pick any exact sequence $0 \rightarrow \operatorname{Sym}^{2} L_{1} \rightarrow ? \rightarrow \Omega \rightarrow 0$ and summate it with the above two. The result is an exact sequence $0 \rightarrow \mathrm{Sym}^{2} L \rightarrow ? \rightarrow \Omega \rightarrow 0$, the corresponding $P_{k, n}$-torsor induces a $P_{k}$-torsor lying in the fibre under consideration.

Set $\operatorname{Bun}_{Q_{k, n}}=\operatorname{Bun}_{P\left(G_{n-k}\right)} \times \operatorname{Bun}_{G_{n-k}} \operatorname{Bun}_{Q_{k}}$, where $P\left(G_{n-k}\right) \subset G_{n-k}$ is the Siegel parabolic. So, $\operatorname{Bun}_{Q_{k, n}}$ classifies a point $0 \rightarrow L_{1} \rightarrow L_{-1} \rightarrow L_{-1} / L_{1} \rightarrow 0$ of $\operatorname{Bun}_{Q_{k}}$ together 
with a Lagrangian subbundle $L / L_{1} \subset L_{-1} / L_{1}$. Consider the diagram

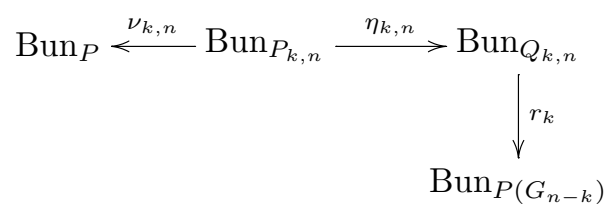

where $r_{k}$ and $\eta_{k, n}$ denote the projections.

Write $S_{P\left(G_{n}\right), \psi}$ to express the dependence of $S_{P, \psi}$ on $n$. Note that $\operatorname{Bun}_{P\left(G_{0}\right)}=\operatorname{Spec} k$, $S_{P\left(G_{0}\right), \psi, g}=\overline{\mathbb{Q}}_{\ell}$ and $S_{P\left(G_{0}\right), \psi, s}=0$.

PROPOSITION 8. - We have a canonical isomorphism commuting with $S_{2}$-action

$$
\left(\eta_{k, n}\right) ! \nu_{k, n}^{*} S_{P, \psi} \underset{\rightrightarrows}{\longrightarrow} r_{k}^{*} S_{P\left(G_{n-k}\right), \psi}[a]\left(\frac{a}{2}\right)
$$

where $a \in \mathbb{Z}$ is the function of a connected component of $\operatorname{Bun}_{Q_{k, n}}$ given by

$$
a=\operatorname{dim} \operatorname{Bun}_{n}-\operatorname{dim} \operatorname{Bun}_{n-k}-\chi\left(L_{1}\right)+\chi\left(\Omega^{-1} \otimes \operatorname{Sym}^{2} L_{1}\right)-\chi\left(\Omega^{-1} \otimes L_{1} \otimes\left(L / L_{1}\right)\right) .
$$

Proof. - Consider the map

$$
\mathcal{X} \times{ }_{\mathrm{Bun}_{P}} \operatorname{Bun}_{P_{k, n}}=\mathcal{V} \times \mathrm{Bun}_{n} \operatorname{Bun}_{P_{k, n}} \stackrel{\operatorname{id} \times \eta_{k, n}}{\longrightarrow} \mathcal{V} \times \operatorname{Bun}_{n} \operatorname{Bun}_{Q_{k, n}}
$$

Write $\mathbb{A}^{1} \stackrel{q_{n}}{\longleftarrow} \mathcal{X}_{G_{n}} \stackrel{p_{n}}{\longrightarrow} \operatorname{Bun}_{P\left(G_{n}\right)}$ to express the dependence on $n$ of the diagram $\mathbb{A}^{1} \stackrel{q}{\longleftarrow} \mathcal{X} \stackrel{p}{\longrightarrow}$ Bun $_{P}$ introduced in Section 5.2.

Denote temporary by $i: \mathcal{X}_{G_{n-k}} \times_{\operatorname{Bun}_{P\left(G_{n-k}\right)}} \operatorname{Bun}_{Q_{k, n}} \hookrightarrow \mathcal{V} \times_{\operatorname{Bun}_{n}} \operatorname{Bun}_{Q_{k, n}}$ the closed embedding given by the condition that $s \in \operatorname{Hom}(L, \Omega)$ lies in $\operatorname{Hom}\left(L / L_{1}, \Omega\right)$.

Set $a_{0}=-\chi\left(\Omega^{-1} \otimes \operatorname{Sym}^{2} L_{1}\right)$ viewed as a function of a connected component of $\operatorname{Bun}_{Q_{k, n}}$. Let us establish a canonical isomorphism

$$
\left(\mathrm{id} \times \eta_{k, n}\right)_{!}\left(q^{*} \mathcal{L}_{\psi} \otimes \overline{\mathbb{Q}}_{\ell}\right) \underset{\rightarrow}{\hookrightarrow} i_{!}\left(q_{n-k}^{*} \mathcal{L}_{\psi} \otimes \overline{\mathbb{Q}}_{\ell}\right)\left[-2 a_{0}\right]\left(-a_{0}\right) .
$$

Consider a $k$-point of $\mathcal{V} \times{ }_{\mathrm{Bun}_{n}} \operatorname{Bun}_{P_{k, n}}$ given by $\left(L_{1} \subset L \subset L_{-1} \subset M\right)$ and $s \in \operatorname{Hom}(L, \Omega)$. The fibre, say $Y$, of id $\times \eta_{k, n}$ over its image in $\mathcal{V} \times \operatorname{Bun}_{n} \operatorname{Bun}_{Q_{k, n}}$ identifies with the stack of exact sequences

$$
0 \rightarrow \mathrm{Sym}^{2} L_{1} \rightarrow ? \rightarrow \Omega \rightarrow 0
$$

on $X$. The restriction of $q^{*} \mathcal{L}_{\psi} \otimes \overline{\mathbb{Q}}_{\ell}$ to $\mathrm{Y}$ is (up to a tensoring by a 1-dimensional vector space) the restriction of $\mathcal{L}_{\psi}$ under the map $Y \rightarrow \mathbb{A}^{1}$ pairing $\operatorname{Sym}^{2} L_{1} \hookrightarrow \operatorname{Sym}^{2} L \stackrel{s \otimes s}{\longrightarrow} \Omega^{2}$ with (20).

So, the fibre of the LHS of (19) vanishes unless $s \in \operatorname{Hom}\left(L / L_{1}, \Omega\right)$. The isomorphism (19) follows, here $a_{0}=\operatorname{dim} Y$.

For the projection pr: $\mathcal{V} \times_{\mathrm{Bun}_{n}} \operatorname{Bun}_{Q_{k, n}} \rightarrow \operatorname{Bun}_{Q_{k, n}}$ we get

$$
\operatorname{pr}_{!}\left(\operatorname{id} \times \eta_{k, n}\right)_{!}\left(q^{*} \mathcal{L}_{\psi} \otimes \overline{\mathbb{Q}}_{\ell}\right) \otimes \overline{\mathbb{Q}}_{\ell}[1]\left(\frac{1}{2}\right)^{\otimes \operatorname{dim} \mathcal{X}} \underset{\rightarrow}{\longrightarrow}\left(\eta_{k, n}\right) ! \nu_{k, n}^{*} S_{P, \psi}
$$

Our assertion follows, because $a=\operatorname{dim} \mathcal{X}_{G_{n}}-\operatorname{dim} \mathcal{X}_{G_{n-k}}-2 a_{0}$.

$4^{\text {e }}$ SÉRIE - TOME $39-2006-\mathrm{N}^{\circ} 3$ 
Proof of Theorem 2. - We have the diagram

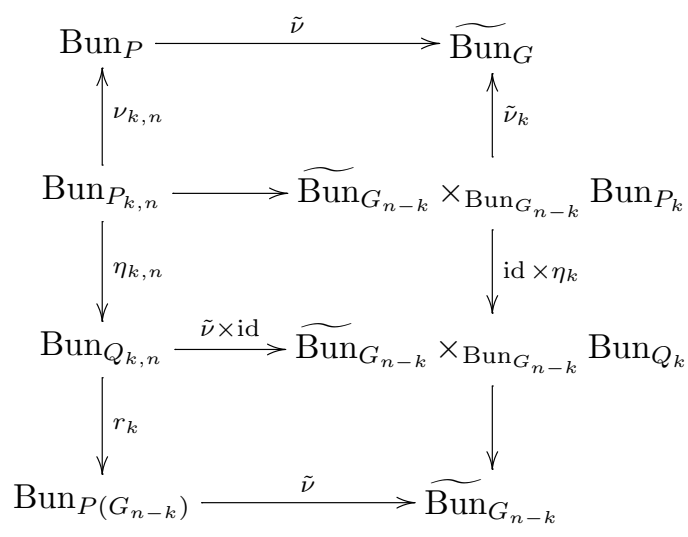

where the middle square is Cartesian. So,

$$
(\tilde{\nu} \times \mathrm{id})^{*}\left(\mathrm{id} \times \eta_{k}\right) ! \tilde{\nu}_{k}^{*} \text { Aut } \widetilde{\rightarrow}\left(\eta_{k, n}\right) ! \nu_{k, n}^{*} \tilde{\nu}^{*} \text { Aut } .
$$

Let ${ }^{0} \operatorname{Bun}_{Q_{k, n}} \subset \operatorname{Bun}_{Q_{k, n}}$ be the open substack given by three conditions: $\mathrm{H}^{0}\left(X, \operatorname{Sym}^{2} L_{1}\right)=0, \mathrm{H}^{0}\left(X, L_{1} \otimes L / L_{1}\right)=0$, and $\mathrm{H}^{0}\left(X, \operatorname{Sym}^{2}\left(L / L_{1}\right)\right)=0$. As in Lemma 6 , one checks that

$$
{ }^{0} \operatorname{Bun}_{Q_{k, n}} \stackrel{\tilde{\nu} \times \mathrm{id}}{\longrightarrow} \widetilde{\operatorname{Bun}}_{G_{n-k}} \times \operatorname{Bun}_{G_{n-k}}{ }^{0} \operatorname{Bun}_{Q_{k}}
$$

is smooth and surjective. Since $\eta_{k, n}^{-1}\left({ }^{0} \operatorname{Bun}_{Q_{k, n}}\right) \subset \nu_{k, n}^{-1}\left({ }^{0} \operatorname{Bun}_{P}\right)$, from Propositions 7 and 8 we get

$$
(\tilde{\nu} \times \mathrm{id})^{*}\left(\mathrm{id} \times \eta_{k}\right) ! \tilde{\nu}_{k}^{*} \text { Aut } \underset{\rightarrow}{\rightarrow} r_{k}^{*} S_{P\left(G_{n-k}\right), \psi} \otimes \overline{\mathbb{Q}}_{\ell}[1]\left(\frac{1}{2}\right)^{\otimes d_{G}-\operatorname{dim} \operatorname{Bun}_{P}+a}
$$

over ${ }^{0} \operatorname{Bun}_{Q_{k, n}}$. The restriction of $r_{k}$ to ${ }^{0} \mathrm{Bun}_{Q_{k, n}}$ factors as

$$
{ }^{0} \operatorname{Bun}_{Q_{k, n}} \stackrel{r_{k}}{\longrightarrow}{ }^{0} \operatorname{Bun}_{P\left(G_{n-k}\right)} \hookrightarrow \operatorname{Bun}_{P\left(G_{n-k}\right)} .
$$

So, by Proposition 7 applied to $G_{n-k}$, the RHS of (22) identifies with

$$
(\tilde{\nu} \times \mathrm{id})^{*}\left(\operatorname{Aut} \otimes \overline{\mathbb{Q}}_{\ell}\right) \otimes \overline{\mathbb{Q}}_{\ell}[1]\left(\frac{1}{2}\right)^{\otimes d_{G}-\operatorname{dim} \operatorname{Bun}_{P}+a+\operatorname{dim} \operatorname{Bun}_{P\left(G_{n-k}\right)}-d_{G_{n-k}}} .
$$

We have $b\left(L_{1}\right)=d_{G}-\operatorname{dim} \operatorname{Bun}_{P}+a+\operatorname{dim} \operatorname{Bun}_{P\left(G_{n-k}\right)}-d_{G_{n-k}}$. Since $\operatorname{Bun}_{Q_{k}} \rightarrow \operatorname{Bun}_{G_{n-k}}$ is smooth, Aut $\otimes \overline{\mathbb{Q}}_{\ell}$ is a shifted perverse sheaf on $\overline{\operatorname{Bun}}_{G_{n-k}} \times{ }_{\operatorname{Bun}_{G_{n-k}}} \operatorname{Bun}_{Q_{k}}$.

Since the restriction of the map (21) to each connected component of ${ }^{0} \mathrm{Bun}_{Q_{k, n}}$ has connected fibres, we get the desired isomorphism.

The second assertion follows from Remark 4 combined with Proposition 8.

\section{Towards geometric $\theta$-lifting}

This section is not used in the proofs and may be skipped. Let $\tau_{n, m}: \operatorname{Bun}_{G_{n}} \times \operatorname{Bun}_{\mathrm{SO}_{m}} \rightarrow$ $\operatorname{Bun}_{G_{n m}}$ be the following map. Given $\mathrm{SO}_{m}$-torsor $\mathcal{F}_{W}$, let $W$ denote the vector bundle induced 
from it via the standard representation of $\mathrm{SO}_{m}$. Given in addition $M \in \mathrm{Bun}_{G_{n}}$ we get naturally a symplectic form $\bigwedge^{2}(M \otimes W) \rightarrow \Omega$. The map $\tau_{n, m}$ sends $(M, W)$ to $M \otimes W$.

Let $\mathcal{A}_{\mathrm{SO}_{m}}$ denote the (naturally $\mathbb{Z} / 2 \mathbb{Z}$-graded) line bundle on $\mathrm{Bun}_{\mathrm{SO}_{m}}$, whose fibre at $\mathcal{F}_{W}$ is $\operatorname{det} \mathrm{R} \Gamma(X, W)$. Write $\mathcal{A}_{G_{n}}$ to express the dependence on $n$ of the determinant of cohomology on $\operatorname{Bun}_{G_{n}}$.

LEMMA 8.-For $m \geqslant 3$ we have a $\mathbb{Z} / 2 \mathbb{Z}$-graded canonical isomorphism over $\operatorname{Bun}_{G_{n}} \times \operatorname{Bun}_{\mathrm{SO}_{m}}$

$$
\tau_{n, m}^{*} \mathcal{A}_{G_{n m}} \stackrel{\longrightarrow}{\rightarrow}\left(\mathcal{A}_{G_{n}}^{m} \otimes \mathcal{A}_{\mathrm{S} \mathbb{O}_{m}}^{2 n}\right) \otimes \operatorname{det} \mathrm{R} \Gamma(X, \mathcal{O})^{\otimes-2 n m} .
$$

Proof. - Step 1. Let us show that for any $M \in \mathrm{Bun}_{G_{n}}, V \in \mathrm{Bun}_{\mathrm{SL}_{2}}$ we have canonically

$$
\operatorname{det} \mathrm{R} \Gamma(X, M \otimes V) \stackrel{\hookrightarrow}{\rightarrow} \operatorname{det} \mathrm{R} \Gamma(X, M)^{2} \otimes \operatorname{det} \mathrm{R} \Gamma(X, V)^{2 n} \otimes \operatorname{det} \mathrm{R} \Gamma(X, \mathcal{O})^{-4 n} .
$$

Indeed, for $V=\mathcal{O}^{2}$ we have $\operatorname{det} \mathrm{R} \Gamma(X, M \otimes V) \stackrel{\rightarrow}{\rightarrow} \operatorname{det} \mathrm{R} \Gamma(X, M)^{2}$. Further, for $M=\mathcal{O}^{n} \oplus \Omega^{n}$

$$
\operatorname{det} \mathrm{R} \Gamma(X, M \otimes V) \widetilde{\rightarrow} \operatorname{det} \mathrm{R} \Gamma(X, V)^{n} \otimes \operatorname{det} \mathrm{R} \Gamma(X, V \otimes \Omega)^{n} \underset{\rightarrow}{\hookrightarrow} \operatorname{det} \mathrm{R} \Gamma(X, V)^{2 n} .
$$

Since $\mathrm{H}^{0}\left(\operatorname{Bun}_{G_{n}}, \mathcal{O}\right)=\mathrm{H}^{0}\left(\operatorname{Bun}_{\mathrm{SL}_{2}}, \mathcal{O}\right)=k$, the assertion follows.

Step 2. Let $\mathcal{F}_{W}^{0}$ be the trivial $\mathrm{SO}_{m}$-torsor on $X$. Restricting $\tau_{n, m}^{*} \mathcal{A}_{G_{n m}}$ under $\operatorname{Bun}_{G_{n}} \stackrel{\operatorname{id} \times \mathcal{F}_{W}^{0}}{\longrightarrow}$ $\operatorname{Bun}_{G_{n}} \times \operatorname{Bun}_{\mathrm{SO}_{m}}$, we get $\mathcal{A}_{G_{n}}^{m}$ canonically.

For $a \in \mathbb{Z} / 2 \mathbb{Z}$ denote by $\mathrm{Bun}_{\mathrm{SO}_{m}}^{a}$ the corresponding connected component of $\mathrm{Bun}_{\mathrm{SO}_{m}}$. Let $\mathcal{F}_{G_{n}}^{0}$ be the $G_{n}$-bundle $\mathcal{O}^{n} \oplus \Omega^{n}$ on $X$. The restriction of $\tau_{n, m}^{*} \mathcal{A}_{G_{n m}}$ under $\mathcal{F}_{G_{n}}^{0} \times \mathrm{id}: \operatorname{Bun}_{\mathrm{SO}_{m}} \rightarrow \operatorname{Bun}_{G_{n}} \times \operatorname{Bun}_{\mathrm{SO}_{m}}$ is $\mathcal{A}_{\mathrm{SO}_{m}}^{2 n}$ canonically. This yields the desired isomorphism over $\operatorname{Bun}_{G_{n}} \times \mathrm{Bun}_{\mathrm{SO}_{m}}^{0}$.

If $\mathcal{E}$ is a line bundle on $X$ of odd degree then $W=\mathcal{E} \oplus \mathcal{E}^{*} \oplus \mathcal{O}^{m-2} \in \operatorname{Bun}_{\mathrm{SO}_{m}}^{1}$. For this $W$ we get

$$
\operatorname{det} \mathrm{R} \Gamma(X, M \otimes W) \stackrel{\rightrightarrows}{\rightarrow} \operatorname{det} \mathrm{R} \Gamma\left(M \otimes\left(\mathcal{E} \oplus \mathcal{E}^{*}\right)\right) \otimes \operatorname{det} \mathrm{R} \Gamma(X, M)^{m-2} .
$$

By step 1,

$$
\operatorname{det} \mathrm{R} \Gamma\left(M \otimes\left(\mathcal{E} \oplus \mathcal{E}^{*}\right)\right) \stackrel{\sim}{\rightarrow} \operatorname{det} \mathrm{R} \Gamma(X, M)^{2} \otimes \operatorname{det} \mathrm{R} \Gamma\left(X, \mathcal{E} \oplus \mathcal{E}^{*}\right)^{2 n} \otimes \operatorname{det} \mathrm{R} \Gamma(X, \mathcal{O})^{-4 n}
$$

The desired isomorphism over $\operatorname{Bun}_{G_{n}} \times \operatorname{Bun}_{\mathrm{SO}_{m}}^{1}$ follows.

By the lemma combined with 3.1.2, for $m$ even there is a canonical map

$$
\tilde{\tau}_{n, m}: \operatorname{Bun}_{G_{n}} \times \operatorname{Bun}_{\mathrm{SO}_{m}} \rightarrow \widetilde{\operatorname{Bun}}_{G_{n m}}
$$

extending $\tau_{n, m}$. For $m$ odd there is a canonical map

$$
\tilde{\tau}_{n, m}: \widetilde{\operatorname{Bun}}_{G_{n}} \times \operatorname{Bun}_{\mathrm{SO}_{m}} \rightarrow \widetilde{\operatorname{Bun}}_{G_{n m}}
$$

extending $\tau_{n, m}$.

The complex $\tilde{\tau}_{n, m}^{*}$ Aut viewed as a kernel of integral operators gives rise to a pair of functors between the categories $\mathrm{D}\left(\widetilde{\operatorname{Bun}}_{G_{n}}\right)$ and $\mathrm{D}\left(\mathrm{Bun}_{\mathrm{SO}_{m}}\right)$ (for $m$ even one may replace $\widetilde{\mathrm{Bun}}_{G_{n}}$ by $\operatorname{Bun}_{G_{n}}$ ). These functors are the geometric counterpart of the classical theta-lifting (in the nonramified case) for the dual reductive pair $\mathbb{S p}_{2 n}, \mathrm{SO}_{m}$ (cf., for example, [22, Section 8]), we will study them separately.

$4^{\text {e }}$ SÉRIE - TOME $39-2006-\mathrm{N}^{\circ} 3$ 


\section{Genuine spherical sheaves on $\widetilde{\mathrm{Gr}}_{G}$}

8.1. Let $\mathcal{O}=k[[t]]$ and $K=k((t))$. Let $\Omega_{\mathcal{O}}$ denote the completed module of relative differentials of $\mathcal{O}$ over $k$. Pick a free $\mathcal{O}$-module $M_{0}$ of rank $2 n$ with symplectic form $\bigwedge^{2} M_{0} \rightarrow \Omega_{\mathcal{O}}$.

In Sections 8.1-8.2 $G$ will denote the sheaf of automorphisms of $M_{0}$ preserving the symplectic form. One associates to $G$ the affine Grassmanian $\mathrm{Gr}_{G}$ (cf. [20] or [12]), which is an ind-scheme over $k$, the fpqc quotient $\operatorname{Gr}_{G}=G(K) / G(\mathcal{O})$. Here $G(\mathcal{O})$ (respectively, $G(K)$ ) is the functor associating to a $k$-algebra $R$ the group of automorphisms of $M_{0, R}:=M_{0} \otimes_{\mathcal{O}} R[[t]]$ (respectively, of $\left.M_{0} \otimes_{\mathcal{O}} R((t))\right)$ preserving all the structures.

Recall that the Picard group of $\operatorname{Gr}_{G}$ is $\mathbb{Z}$ (cf. [12]), let us introduce the notation for the generator. We have the affine Grassmanian $\operatorname{Gr}_{\mathrm{SL}\left(M_{0}\right)}$. Its $R$-points are projective $R[[t]]$-modules of finite type $M \subset M_{0} \otimes_{\mathcal{O}} R((t))$ with

- $t^{m} M_{0, R} \subset M \subset t^{-m} M_{0, R}$ for some $m \gg 0$;

- $\operatorname{det}_{R[[t]]} M=\operatorname{det}_{R[[t]]} M_{0, R}$ as a subspace of $\left(\operatorname{det}_{R[t]]} M_{0, R}\right) \otimes_{R[[t]]} R((t))$.

We postpone to Lemma 9 the proof of the fact that $M / t^{m} M_{0, R}$ is a projective $R$-module for $m \gg 0$. This allows to introduce the line bundle $\mathcal{L}$ on $\operatorname{Gr}_{\mathrm{SL}\left(M_{0}\right)}$ whose fibre at $M$ is

$$
\operatorname{det}\left(M_{0}: M\right):=\operatorname{det}_{R}\left(M_{0} / t^{m} M_{0}\right) \otimes \operatorname{det}_{R}\left(M / t^{m} M_{0}\right)^{-1},
$$

independent of $m$ such that $t^{m} M_{0} \subset M$. View it as $\mathbb{Z} / 2 \mathbb{Z}$-graded purely of degree zero.

The standard representation of $G$ yields a map $\operatorname{Gr}_{G} \rightarrow \operatorname{Gr}_{\mathrm{SL}\left(M_{0}\right)}$, and we also write $\mathcal{L}$ for the restriction of this line bundle to $\mathrm{Gr}_{G}$. Then $\mathcal{L}$ generates the Picard group of $\mathrm{Gr}_{G}$. Recall that $\mathcal{L}$ is $G(\mathcal{O})$-equivariant on $\mathrm{Gr}_{G}$. Let $\widetilde{G r}_{G} \rightarrow \mathrm{Gr}_{G}$ denote the $\mu_{2}$-gerbe of square roots of $\mathcal{L}$. Then $G(\mathcal{O})$ acts on $\widetilde{G r}_{G}$ extending the action on $\operatorname{Gr}_{G}$ (cf. A.3).

Definition 4. - Let $\operatorname{Sph}\left(\widetilde{\mathrm{Gr}}_{G}\right)$ be the category of $G(\mathcal{O})$-equivariant perverse sheaves on $\widetilde{\mathrm{Gr}}_{G}$ on which $-1 \in \mu_{2}$ acts as -1 . We call it the category of genuine spherical sheaves on $\widetilde{\mathrm{Gr}}_{G}$.

A $\theta$-characteristic is a free $\mathcal{O}$-module $\mathcal{N}$ of rank 1 together with $\mathcal{N} \otimes_{\mathcal{O}} \mathcal{N} \leftrightharpoons \Omega_{\mathcal{O}}$. A choice of a $\theta$-characteristic yields an isomorphism of group schemes $G(\mathcal{O}) \widetilde{S p}\left(M_{0} \otimes_{\mathcal{O}} \mathcal{N}^{-1}\right)$ over $k$. A further choice of a symplectic base in $M_{0} \otimes_{\mathcal{O}} \mathcal{N}^{-1}$ over $\mathcal{O}$ identifies $G(\mathcal{O})$ with $\mathbb{S p}_{2 n}(\mathcal{O})$. So, we may view the standard maximal torus and Borel $T \subset B \subset \mathbb{S p}_{2 n} \subset \mathbb{S p}_{2 n}(\mathcal{O})$ as subgroups of $G(\mathcal{O})$. Write $\Lambda^{+}$for the set of dominant coweights of $\mathbb{S p}_{2 n}$.

We have a stratification of $\mathrm{Gr}_{G}$ by $G(\mathcal{O})$-orbits indexed by $\Lambda^{+}$, write $\operatorname{Gr}_{G}^{\lambda}$ for the $G(\mathcal{O})$-orbit passing by $\lambda(t) \in T(K)$. Let $\widetilde{\mathrm{Gr}}_{G}^{\lambda}$ be the preimage of $\mathrm{Gr}_{G}^{\lambda}$ in $\widetilde{\mathrm{Gr}}_{G}$.

Proposition 9.- For any $\lambda \in \Lambda^{+}$there is a $G(\mathcal{O})$-equivariant trivialization $\widetilde{\mathrm{Gr}}_{G}^{\lambda} \underset{\rightrightarrows}{\longrightarrow}$ $\operatorname{Gr}_{G}^{\lambda} \times B\left(\mu_{2}\right)$, the $G(\mathcal{O})$-action on the RHS being the product of the action on $\operatorname{Gr}_{G}^{\lambda}$ and the trivial action on $B\left(\mu_{2}\right)$.

Proof. - Step 1. For $\lambda \in \Lambda^{+}$denote by $\mathrm{St}_{\lambda}$ the stabilizer of $\lambda(t) \in \operatorname{Gr}_{G}$ in $G(\mathcal{O})$. Let $M_{\lambda}=\lambda(t) M_{0}$ and $M^{\prime}=M_{0}+M_{\lambda}, M^{\prime \prime}=M_{0} \cap M_{\lambda}$.

The symplectic form $\bigwedge^{2}\left(M_{0} \otimes_{\mathcal{O}} K\right) \rightarrow \Omega(K)=\Omega_{\mathcal{O}} \otimes_{\mathcal{O}} K$ induces a map $\left(M^{\prime} / M_{0}\right) \otimes$ $\left(M^{\prime} / M_{\lambda}\right) \stackrel{\rightarrow}{\rightarrow}\left(M_{\lambda} / M^{\prime \prime}\right) \otimes\left(M_{0} / M^{\prime \prime}\right) \rightarrow \Omega(K) / \Omega_{\mathcal{O}}$. Composing further with the residue map, we get a pairing between $k$-vector spaces $M^{\prime} / M_{0}$ and $M^{\prime} / M_{\lambda}$. We will check in step 2 that the pairing is perfect. So, the fibre of $\mathcal{L}$ at $M_{\lambda}$ is

$$
\mathcal{L}_{M_{\lambda}} \widetilde{\rightarrow} \operatorname{det}\left(M_{0}: M_{\lambda}\right) \underset{\rightarrow}{\widetilde{d e t}\left(M^{\prime} / M_{\lambda}\right)} \underset{\operatorname{det}\left(M^{\prime} / M_{0}\right)}{\longrightarrow} \operatorname{det}\left(M^{\prime} / M_{\lambda}\right)^{\otimes 2}
$$


The group $\mathrm{St}_{\lambda}$ acts on $\operatorname{det}\left(M^{\prime} / M_{\lambda}\right)$ by some character $\chi: \mathrm{St}_{\lambda} \rightarrow \mathbb{G}_{m}$. So, $\mathrm{St}_{\lambda}$ acts on $\mathcal{L}_{M_{\lambda}}$ by $\chi^{2}$. Let $\mathcal{B}$ be the $G(\mathcal{O})$-equivariant line bundle on $\operatorname{Gr}_{G}^{\lambda}$ corresponding to $\chi$. Then we have a $G(\mathcal{O})$-equivariant isomorphism $\left.\mathcal{B}^{2} \widetilde{\mathcal{L}}\right|_{\mathrm{Gr}_{G}^{\lambda}}$, and our assertion follows from Lemma 17 .

Step 2. Realize $\mathbb{S p}_{2 n}$ as the subgroup of $\mathrm{SL}_{2 n}$ preserving the form on $k^{2 n}$ given by the matrix

$$
\left(\begin{array}{cc}
0 & E_{n} \\
-E_{n} & 0
\end{array}\right)
$$

where $E_{n}$ is the identity matrix in $\mathrm{SL}_{n}$. Let $T \subset \mathbb{S p}_{2 n}$ be the maximal torus of diagonal matrices. A coweight $\lambda=\left(a_{1}, \ldots, a_{n} ;-a_{1}, \ldots,-a_{n}\right)$ of $T$ is dominant iff $a_{1} \geqslant \cdots \geqslant a_{n} \geqslant 0$. Pick a trivialization $\mathcal{N} \cong \mathcal{O}$ and a symplectic base $e_{i}$ in $M_{0}$. Then

$$
M_{\lambda}=t^{a_{1}} \mathcal{O} e_{1} \oplus \cdots \oplus t^{a_{n}} \mathcal{O} e_{n} \oplus t^{-a_{1}} \mathcal{O} e_{n+1} \oplus \cdots \oplus t^{-a_{n}} \mathcal{O} e_{2 n}
$$

and $M^{\prime}=\mathcal{O} e_{1} \oplus \cdots \oplus \mathcal{O} e_{n} \oplus t^{-a_{1}} \mathcal{O} e_{n+1} \oplus \cdots \oplus t^{-a_{n}} \mathcal{O} e_{2 n}$. Since

$$
\begin{aligned}
& M^{\prime} / M_{0} \underset{\rightarrow}{\rightarrow} t^{-a_{1}} \mathcal{O} e_{n+1} \oplus \cdots \oplus t^{-a_{n}} \mathcal{O} e_{2 n} / \mathcal{O} e_{n+1} \oplus \cdots \oplus \mathcal{O} e_{2 n}, \\
& M^{\prime} / M_{\lambda} \widetilde{\longrightarrow} \mathcal{O} e_{1} \oplus \cdots \oplus \mathcal{O} e_{n} / t^{a_{1}} \mathcal{O} e_{1} \oplus \cdots \oplus t^{a_{n}} \mathcal{O} e_{n},
\end{aligned}
$$

the pairing is perfect.

Let $W$ denote the nontrivial local system of rank one on $B\left(\mu_{2}\right)$ corresponding to the covering Spec $k \rightarrow B\left(\mu_{2}\right)$. For $\lambda \in \Lambda^{+}$there is a unique irreducible $G(\mathcal{O})$-equivariant perverse sheaf on $\widetilde{\mathrm{Gr}}_{G}^{\lambda}$, on which $-1 \in \mu_{2}$ acts as -1 , namely $\left(\overline{\mathbb{Q}}_{\ell} \otimes W\right) \otimes \overline{\mathbb{Q}}_{\ell}[1]\left(\frac{1}{2}\right)^{\otimes \operatorname{dim} \operatorname{Gr}_{G}^{\lambda}}$. Denote by $\mathcal{A}_{\lambda}$ its Goresky-MacPherson extension to $\widetilde{\mathrm{Gr}}_{G}$. By Proposition 9, the irreducible objects of the category $\operatorname{Sph}\left(\widetilde{\mathrm{Gr}}_{G}\right)$ are exactly $\mathcal{A}_{\lambda}, \lambda \in \Lambda^{+}$.

Note that $\operatorname{Sph}\left(\widetilde{\mathrm{Gr}}_{G}\right)$ is closed under extensions in $\mathrm{P}\left(\widetilde{\mathrm{Gr}}_{G}\right)$ (if $-1 \in \mu_{2}$ acts as -1 on perverse sheaves $K_{1}, K_{2}$ then it acts as -1 on any extension of $K_{1}$ by $\left.K_{2}\right)$. Since $\mathbb{D}\left(\mathcal{A}_{\lambda}\right) \widetilde{\sim} \mathcal{A}_{\lambda}$ canonically, $\operatorname{Sph}\left(\widetilde{\mathrm{Gr}}_{G}\right)$ is preserved by Verdier duality.

Consider the action of the torus $T \subset G(\mathcal{O})$ on $\mathrm{Gr}_{G}$. The following will be used in Section 8.4.

LEMMA 9. -

(i) There is a covering of $\mathrm{Gr}_{G}$ by $T$-invariant open ind-schemes $U_{i}$ and T-equivariant

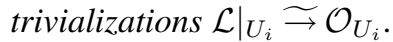

(ii) For an R-point $M \subset M_{0} \otimes_{\mathcal{O}} R((t))$ of $\mathrm{Gr}_{\mathrm{SL}\left(M_{0}\right)}$ and $m \gg 0$ the $R$-module $M / t^{m} M_{0, R}$ is projective.

Proof. - (i) Pick a trivialization $\mathcal{N} \widetilde{\mathcal{O}}$, so that our base of $M_{0} \otimes \mathcal{N}^{-1}$ gives rise to a base $\left\{e_{1}, \ldots, e_{2 n}\right\}$ of $M_{0}$. Consider the corresponding maximal torus $T^{\prime}$ of $\operatorname{SL}\left(M_{0}\right)$. Set $M^{-}=A e_{1} \oplus \cdots \oplus A e_{2 n}$ with $A=t^{-1} k\left[t^{-1}\right]$. For a coweight $\lambda: \mathbb{G}_{m} \rightarrow T^{\prime}$ of $\operatorname{SL}\left(M_{0}\right)$ denote by $U_{\lambda} \subset \operatorname{Gr}_{\mathrm{SL}\left(M_{0}\right)}$ the open locus classifying lattices $M \subset M_{0} \otimes_{\mathcal{O}} K$ such that $M \oplus \lambda(t) M^{-}=$ $M_{0} \otimes_{\mathcal{O}} K$. Here $\lambda=\left(b_{1}, \ldots, b_{2 n}\right)$ with $\sum b_{i}=0$ and $\lambda(t) M^{-}=A t^{b_{1}} e_{1} \oplus \cdots \oplus A t^{b_{2 n}} e_{2 n}$.

One checks that the union of $U_{\lambda}$ is $\operatorname{Gr}_{\mathrm{SL}\left(M_{0}\right)}$. Clearly, $U_{\lambda}$ is $T^{\prime}$-invariant. As shown by Faltings [12, Section 2], for each $\lambda$ there is a trivialization $\left.\mathcal{L}\right|_{U_{\lambda}} \stackrel{\sim}{\rightarrow} \mathcal{O}_{U_{\lambda}}$ equivariant under the stabilizer of $\lambda(t) M^{-}$in $\operatorname{SL}\left(M_{0}\right)(K)$. This stabilizer contains $T^{\prime}$, so the trivializations are $T^{\prime}$-equivariant.

Restricting everything under the map $\mathrm{Gr}_{G} \rightarrow \mathrm{Gr}_{\mathrm{SL}\left(M_{0}\right)}$ corresponding to the standard representation, one concludes the proof.

(ii) (argument due to the unknown referee) Localizing in Zariski topology of $R$, pick a coweight $\lambda$ of $\mathrm{SL}\left(M_{0}\right)$ such that $M \oplus \lambda(t) M_{R}^{-}=M_{0} \otimes_{\mathcal{O}} R((t))$. Here 
$M_{R}^{-}=A_{R} e_{1} \oplus \cdots \oplus A_{R} e_{2 n}$ and $A_{R}=t^{-1} R\left[t^{-1}\right]$. For $m \gg 0$ we get $t^{-m} M_{0, R}=M \oplus U$, where $U=\lambda(t) M_{R}^{-} \cap t^{-m} M_{0, R}$, and

$$
\left(M / t^{m} M_{0, R}\right) \oplus U \widetilde{\rightarrow} t^{-m} M_{0, R} / t^{m} M_{0, R} .
$$

\subsection{The convolution product}

Following [20], consider the diagram

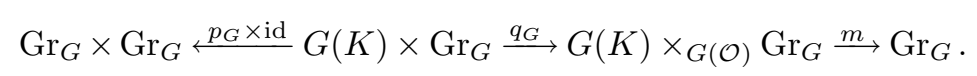

Here $p_{G}: G(K) \rightarrow \mathrm{Gr}_{G}$ is the projection, $G(K) \times_{G(\mathcal{O})} \mathrm{Gr}_{G}$ is the quotient of $G(K) \times \mathrm{Gr}_{G}$ by $G(\mathcal{O})$, where the action is given by $x(g, h G(\mathcal{O}))=\left(g x^{-1}, x h G(\mathcal{O})\right)$ for $x \in G(\mathcal{O})$, and $m$ is the product map.

The map $p_{G} \times m: G(K) \times{ }_{G(\mathcal{O})} \operatorname{Gr}_{G} \rightarrow \operatorname{Gr}_{G} \times \operatorname{Gr}_{G}$ sending $(g, h G(\mathcal{O}))$ to $(g G(\mathcal{O}), g h G(\mathcal{O}))$ is an isomorphism.

We have a canonical isomorphism $q_{G}^{*} m^{*} \mathcal{L} \widetilde{\neg} p_{G}^{*} \mathcal{L} \otimes \mathcal{L}$. Moreover, the above $G(\mathcal{O})$-action on $G(K) \times \operatorname{Gr}_{G}$ lifts to a $G(\mathcal{O})$-equivariant structure on $p_{G}^{*} \mathcal{L} \otimes \mathcal{L}$ giving rise to the line bundle $p_{G}^{*} \mathcal{L} \widetilde{\otimes} \mathcal{L}$ on $G(K) \times_{G(\mathcal{O})} \mathrm{Gr}_{G}$. Thus, $m^{*} \mathcal{L} \leftrightharpoons p_{G}^{*} \mathcal{L} \widetilde{\otimes} \mathcal{L}$ canonically.

Set $\widetilde{G(K)}=G(K) \times_{\mathrm{Gr}_{G}} \widetilde{\mathrm{Gr}}_{G}$. Both actions of $G(\mathcal{O})$ on $G(K)$ by left and right translations extend naturally to actions on $\widehat{G(K)}$. We will refer to them again as actions by left and right translations, by abuse of terminology. Under the action on $\widetilde{G(K)}$ by right translations, the projection $\tilde{p}_{G}: \widetilde{G(K)} \rightarrow \widetilde{\operatorname{Gr}}_{G}$ is a $G(\mathcal{O})$-torsor (cf. A.2).

Taking the tensor product of square roots of $p_{G}^{*} \mathcal{L}$ and of $\mathcal{L}$, we get a map $\tilde{m}$ that fits into the diagram

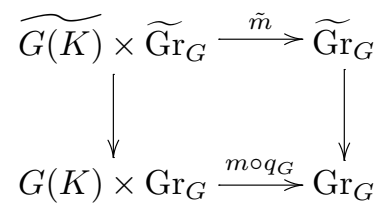

One checks that

$$
\tilde{p}_{G} \times \tilde{m}: \widetilde{G(K)} \times \widetilde{\mathrm{Gr}}_{G} \rightarrow \widetilde{\mathrm{Gr}}_{G} \times \widetilde{\mathrm{Gr}}_{G}
$$

is a $G(\mathcal{O})$-torsor, where $G(\mathcal{O})$ acts on $\widetilde{G(K)} \times \widetilde{\operatorname{Gr}}_{G}$ as the product of the action by right translations on $\widetilde{G(K)}$ with the action on $\widetilde{\mathrm{Gr}}_{G}$.

Consider the diagram

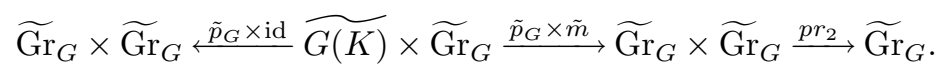

Definition 5.-For $K_{1}, K_{2} \in \operatorname{Sph}\left(\widetilde{\mathrm{Gr}}_{G}\right)$ define the convolution product $K_{1} * K_{2} \in$ $\mathrm{D}\left(\widetilde{\mathrm{Gr}}_{G}\right)$ by

$$
K_{1} * K_{2}=\operatorname{pr}_{2 !} K
$$

where $K$ is a perverse sheaf on $\widetilde{\mathrm{Gr}}_{G} \times \widetilde{\mathrm{Gr}}_{G}$ such that $\left(\tilde{p}_{G} \times \tilde{m}\right)^{*} K \widetilde{p_{G}^{*}} K_{1} \otimes K_{2}$. Since (23) is a $G(\mathcal{O})$-torsor and $\tilde{p}_{G}^{*} K_{1} \otimes K_{2}$ is equivariant under the corresponding $G(\mathcal{O})$-action on $\widetilde{G(K)} \times \widetilde{\mathrm{Gr}}_{G}, K$ is defined up to a unique isomorphism (cf. A.2). 
For $(a, b) \in \mu_{2} \times \mu_{2}$ the image under $\tilde{p}_{G} \times \tilde{m}$ of the corresponding 2-automorphism of $\widetilde{G(K)} \times \widetilde{\mathrm{Gr}}_{G}$ is the 2-automorphism $(a, a b)$ of $\widetilde{\mathrm{Gr}}_{G} \times \widetilde{\mathrm{Gr}}_{G}$. So, by Lemma $16, K$ descends to a perverse sheaf $K^{\prime}$ on $\mathrm{Gr}_{G} \times \widetilde{\mathrm{Gr}}_{G}$ (such $K^{\prime}$ is defined up to a unique isomorphism). Since

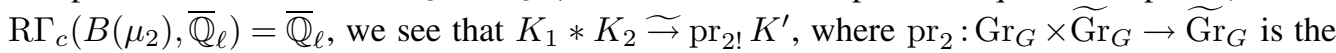
projection. Moreover, $-1 \in \mu_{2}$ acts on $K_{1} * K_{2}$ as -1 .

Proposition 10. - For $K_{1}, K_{2} \in \operatorname{Sph}\left(\widetilde{\mathrm{Gr}}_{G}\right)$ we have $K_{1} * K_{2} \in \operatorname{Sph}\left(\widetilde{\mathrm{Gr}}_{G}\right)$.

Proof. - Following [20], stratify $\mathrm{Gr}_{G} \times \widetilde{\mathrm{Gr}}_{G}$ by locally closed substacks $\widetilde{\mathrm{Gr}}_{G}^{\lambda, \mu}, \lambda, \mu \in \Lambda^{+}$, where $\widetilde{\mathrm{Gr}}_{G}^{\lambda, \mu}$ is the preimage of $\left(p_{G} \times m\right)\left(p_{G}^{-1}\left(\mathrm{Gr}_{G}^{\lambda}\right) \times{ }_{G(\mathcal{O})} \mathrm{Gr}_{G}^{\mu}\right)$ under $\mathrm{Gr}_{G} \times \widetilde{\mathrm{Gr}}_{G} \rightarrow$ $\mathrm{Gr}_{G} \times \mathrm{Gr}_{G}$.

Stratify also $\widetilde{\mathrm{Gr}}_{G}$ by $\widetilde{\mathrm{Gr}}_{G}^{\lambda}, \lambda \in \Lambda^{+}$. By Lemma 4.3 of loc.cit., $\mathrm{pr}_{2}: \mathrm{Gr}_{G} \times \widetilde{\mathrm{Gr}}_{G} \rightarrow \widetilde{\mathrm{Gr}}_{G}$ is a stratified semi-small map. Our assertion follows from Lemma 4.2 of loc.cit.

In a similar way one defines a convolution product $K_{1} * K_{2} * K_{3}$ of three sheaves $K_{1}, K_{2}, K_{3} \in \operatorname{Sph}\left(\widetilde{\operatorname{Gr}}_{G}\right)$. Moreover, $\left(K_{1} * K_{2}\right) * K_{3} \widetilde{\rightarrow} K_{1} * K_{2} * K_{3} \underset{\rightrightarrows}{\widetilde{C}} K_{1} *\left(K_{2} * K_{3}\right)$ canonically, and $\mathcal{A}_{0}$ is a unit object. $\mathrm{So}, \operatorname{Sph}\left(\widetilde{\mathrm{Gr}}_{G}\right)$ is an associative tensor category (a category with tensor functor and an associativity constraint).

Observe that for each $\lambda \in \Lambda^{+}$the $G(\mathcal{O})$-orbit $\operatorname{Gr}_{G}^{\lambda}$ is even-dimensional.

PROPOSITION 11.-

(1) For $\lambda \in \Lambda^{+}$the odd cohomology sheaves of $\mathcal{A}_{\lambda}$ (with respect to the usual $t$-structure) vanish.

(2) The category $\operatorname{Sph}\left(\widetilde{\mathrm{Gr}}_{G}\right)$ is semi-simple.

Proof. - (1a) Given $\lambda_{1}, \ldots, \lambda_{r} \in \Lambda^{+}$, consider the convolution diagram

$$
m: \operatorname{Conv}^{\lambda_{1}, \ldots, \lambda_{r}} \rightarrow \overline{\operatorname{Gr}}_{G}^{\lambda_{1}+\cdots+\lambda_{r}},
$$

where we have set $\operatorname{Conv}^{\lambda_{1}, \ldots, \lambda_{r}}=\operatorname{Gr}_{G}^{\lambda_{1}} \widetilde{\times} \cdots \widetilde{\times} \operatorname{Gr}_{G}^{\lambda_{r}}$. Let $\widetilde{\operatorname{Conv}}{ }^{\lambda_{1}, \ldots, \lambda_{r}}$ be the restriction of the gerbe $\widetilde{\mathrm{Gr}}_{G}$ under the above map $m$. The canonical section $s: \mathrm{Gr}_{G}^{\lambda_{1}+\cdots+\lambda_{r}} \rightarrow \widetilde{\mathrm{Gr}}_{G}^{\lambda_{1}+\cdots+\lambda_{r}}$ yields a section $m^{-1}(s)$ of the gerbe $\widetilde{\operatorname{Conv}}{ }^{\lambda_{1}, \ldots, \lambda_{r}}$ over $m^{-1}\left(\operatorname{Gr}_{G}^{\lambda_{1}+\cdots+\lambda_{r}}\right)$. One checks that this section extends to a section $\mathrm{Conv}^{\lambda_{1}, \ldots, \lambda_{r}} \rightarrow \widetilde{\operatorname{Conv}}^{\lambda_{1}, \ldots, \lambda_{r}}$.

(1b) We adopt Gaitsgory's proof of a theorem of Lusztig to our situation [13, A.7]. Namely, let $\mathcal{F} l$ denote the affine flag variety. This is the ind-scheme classifying a $G$-bundle $\mathcal{F}_{G}$ on $\operatorname{Spec} \mathcal{O}$ with trivialization $\left.\mathcal{F}_{G} \underset{\rightrightarrows}{\rightrightarrows} \mathcal{F}_{G}^{0}\right|_{\text {Spec } K}$ and a reduction of $\left.\mathcal{F}_{G}\right|_{\text {Spec } \mathcal{O} /(t)}$ to the Borel subgroup $B$.

Let $\widetilde{\mathcal{F}} l$ denote the restriction of the gerbe $\widetilde{\mathrm{Gr}}_{G}$ under the (smooth) projection $\mathcal{F} l \rightarrow \mathrm{Gr}_{G}$. Let $I \subset G(\mathcal{O})$ be the Iwahory subgroup. For an element $w$ of the affine Weil group of $G$, let $\mathcal{F} l^{w}$ denote the corresponding $I$-orbit on $\mathcal{F} l$. Set $\widetilde{\mathcal{F} l}{ }^{w}=\mathcal{F} l^{w} \times \mathcal{F} l \widetilde{\mathcal{F}} l$.

Let $\mu \in \Lambda^{+}$be such that the projection $\mathcal{F} l^{w} \rightarrow \mathrm{Gr}_{G}$ factors through $\mathrm{Gr}_{G}^{\mu}$. The canonical

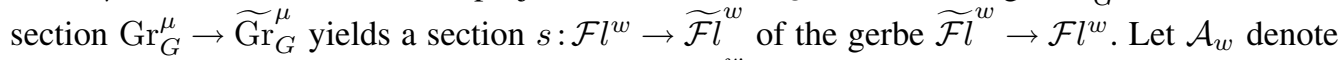
the irreducible perverse sheaf on the closure of $\widetilde{\mathcal{F}}^{w}$ on which $-1 \in \mu_{2}$ acts as -1 and whose restriction under $s$ is $\mathrm{IC}_{\mathcal{F} l^{w}}$. It suffices to show the parity vanishing for stalks of $\mathcal{A}_{w}$.

Let $w=s_{1} \cdot \ldots \cdot s_{r}$ be a reduced decomposition of $w$ into a product of simple reflections. Denote by $p: \operatorname{Conv}_{\mathcal{F} l}^{s_{1}, \ldots, s_{r}} \rightarrow \overline{\mathcal{F} l}^{w}$ the Bott-Samelson resolution (loc.cit. or [12, Section 3], where it is referred to as Demazure resolution). Let $\widetilde{\operatorname{Conv}}_{\mathcal{F l}} s_{1}, \ldots, s_{r}$ be the restriction of our gerbe 
to $\operatorname{Conv}_{\mathcal{F} l}^{s_{1}, \ldots, s_{r}}$. By (1a), the section

$$
p^{-1}\left(\mathcal{F} l^{w}\right) \rightarrow p^{-1}\left(\widetilde{\mathcal{F}} l^{w}\right)
$$

induced by $s$ extends to a global section $\operatorname{Conv}_{\mathcal{F} l}^{s_{1}, \ldots, s_{r}} \rightarrow \widetilde{\operatorname{Conv}}_{\mathcal{F} l} s_{1}, \ldots, s_{r}$. The desired assertion follows, because the fibres of $p$ have cohomology with compact support in even degrees only $[13$, A.7].

(2) Follows from (1) as in [20, Lemma 7.1]. This uses the fact that each $\mathrm{Gr}_{G}^{\lambda}$ has cohomology only in even degrees [20, Section 2].

Remark 6. - The group of automorphisms of the $k$-algebra $\mathcal{O}$ is naturally the group of $k$-points of a (reduced) affine group scheme $\operatorname{Aut}^{0} \mathcal{O}$ over $k$. Assume that $M_{0}=\mathcal{O}^{n} \oplus \Omega_{\mathcal{O}}^{n}$ with standard symplectic form. Then $\mathrm{Aut}^{0} \mathcal{O}$ acts on $M_{0}$ and, hence, on $\mathrm{Gr}_{G}$. Moreover, $\mathcal{L}$ is naturally equivariant under this action. It follows that $\mathrm{Aut}^{0} \mathcal{O}$ acts on $\widetilde{\mathrm{Gr}}_{G}$. Proposition 9 then can be strengthened saying that the gerbe $\widetilde{G r}_{G}^{\lambda} \rightarrow \operatorname{Gr}_{G}^{\lambda}$ admits a $G(\mathcal{O}) \rtimes$ Aut $^{0} \mathcal{O}$-equivariant trivialization.

We also see that each $\mathcal{A}_{\lambda}$ is $G(\mathcal{O}) \rtimes \mathrm{Aut}^{0} \mathcal{O}$-equivariant (this property is true for the constant sheaf over $\operatorname{Gr}_{G}^{\lambda}$ and is preserved under intermediate extension). By Proposition 11, each $K \in \operatorname{Sph}\left(\widetilde{\mathrm{Gr}}_{G}\right)$ is $\mathrm{Aut}^{0} \mathcal{O}$-equivariant. Moreover, such equivariant structure is unique (because the stabilizer of a point is connected) and compatible with any morphism in $\operatorname{Sph}\left(\widetilde{\mathrm{Gr}}_{G}\right)$.

\subsection{The fusion product}

Following [20], we will show that the convolution product defined above can be interpreted as a 'fusion' product, thus leading to a commutativity constraint on $\operatorname{Sph}\left(\widetilde{\mathrm{Gr}}_{G}\right)$. The original idea of this interpretation for spherical sheaves on $\mathrm{Gr}_{G}$ is due to V. Drinfeld.

Let $G$ denote the sheaf of groups on $X$ introduced in Section 3.2. For $x \in X(k)$ write $\mathcal{O}_{x}$ for the completed local ring at $x$ and $K_{x}$ for its fraction field. Write $\operatorname{Gr}_{G, x}=G\left(K_{x}\right) / G\left(\mathcal{O}_{x}\right)$ for the corresponding version of the affine Grassmanian.

Write $\mathcal{F}_{G}^{0}$ for the 'trivial' $G$-torsor on $X$ given by $M_{0}=\mathcal{O}_{X}^{n} \oplus \Omega^{n}$ with standard symplectic form $\bigwedge^{2} M_{0} \rightarrow \Omega$.

For a $k$-algebra $R$ write $X_{R}=X \times \operatorname{Spec} R$ and $X_{R}^{*}=(X-x) \times \operatorname{Spec} R$. By [1,2], $\operatorname{Gr}_{G, x}$ is the functor on the category of $k$-algebras sending $R$ to the set of isomorphism classes of $\left\{\mathcal{F}_{G}, \nu\right\}$, where $\mathcal{F}_{G}$ is a $G$-torsor on $X_{R}$ and $\nu:\left.\mathcal{F}_{G} \widetilde{\rightrightarrows} \mathcal{F}_{G}^{0}\right|_{X_{R}^{*}}$ is a trivialization outside $x$.

Let $M$ denote the vector bundle on $X$ induced from $\mathcal{F}_{G}$ via the standard representation of $G$. Set $M_{x}=M \otimes \mathcal{O}_{x}$ and $M_{0, x}=M_{0} \otimes \mathcal{O}_{x}$. Then $M_{x} \subset M_{0, x} \otimes \mathcal{O}_{x} K_{x}$ is a sublattice, and we continue to denote by $\mathcal{L}$ the line bundle on $\operatorname{Gr}_{G, x}$ with fibre $\operatorname{det}\left(M_{0, x}: M_{x}\right)$. Then $\widetilde{\operatorname{Gr}}_{G, x}$ and $\operatorname{Sph}\left(\widetilde{\operatorname{Gr}}_{G, x}\right)$ are defined as in Section 8.1.

Write $\mathrm{Gr}_{G, X^{d}}$ for the functor associating to a $k$-algebra $R$ the set

$$
\left\{\left(x_{1}, \ldots, x_{d}\right) \in X^{d}(R), \text { a } G \text {-torsor } \mathcal{F}_{G} \text { on } X_{R},\left.\mathcal{F}_{G} \stackrel{\sim}{\rightarrow} \mathcal{F}_{G}^{0}\right|_{X_{R}-\cup x_{i}}\right\} \text {. }
$$

Here $x_{i} \in X(R)$ are thought of as subschemes in $X_{R}$ by taking their graphs.

Let $G_{X^{d}}$ denote the functor sending a $k$-algebra $R$ to the set $\left\{\left(x_{1}, \ldots, x_{d}\right) \in X^{d}(R), \mu\right)$, where $\mu$ is an automorphism of $\mathcal{F}_{G}^{0}$ restricted to the formal neighborhood $\widehat{X}_{R, D}$ of $D=$ $x_{1} \cup \cdots \cup x_{d}$ in $X_{R}$. So, $G_{X^{d}}$ is a group scheme over $X^{d}$, whose fibre over $\left(x_{1}, \ldots, x_{d}\right)$ is $\prod_{i} G\left(\mathcal{O}_{y_{i}}\right)$ with $\left\{y_{1}, \ldots, y_{s}\right\}=\left\{x_{1}, \ldots, x_{d}\right\}$ and $y_{i}$ pairwise distinct.

Let $\mathcal{L}$ be the line bundle on $\operatorname{Gr}_{G, X^{n}}$ whose fibre is $\operatorname{det} \mathrm{R} \Gamma\left(X, M_{0}\right) \otimes \operatorname{det} \mathrm{R} \Gamma(X, M)^{-1}$, where $M$ is the vector bundle on $X$ induced from $\mathcal{F}_{G}$ via the standard representation of $G$. 
Lemma 10. - For a k-point $\left(x_{1}, \ldots, x_{d}, \mathcal{F}_{G}\right)$ of $\operatorname{Gr}_{G, X^{d}}$ let $\left\{y_{1}, \ldots, y_{s}\right\}=\left\{x_{1}, \ldots, x_{d}\right\}$ with $y_{i}$ pairwise distinct. The fibre of $\mathcal{L}$ at this $k$-point is canonically isomorphic (as $\mathbb{Z} / 2 \mathbb{Z}$ graded) to

$$
\bigotimes_{i=1}^{s} \operatorname{det}\left(M_{0, y_{i}}: M_{y_{i}}\right)
$$

One checks that the natural action of $G_{X^{d}}$ on $\mathrm{Gr}_{G, X^{d}}$ lifts to a $G_{X^{d}}$-equivariant structure on $\mathcal{L}$. We have $\widetilde{\mathrm{Gr}}_{G, X^{d}}$ and $\operatorname{Sph}\left(\widetilde{\mathrm{Gr}}_{G, X^{d}}\right)$ defined as above.

8.3.1. Consider the diagram of stacks over $X^{2}$, where the left and right square is Cartesian

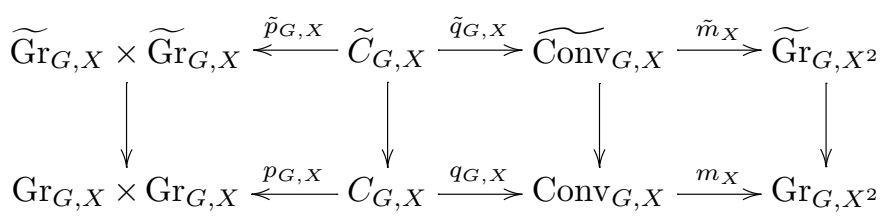

Here the low row is the usual convolution diagram [20, (5.2)]. Namely, $C_{G, X}$ is the ind-scheme classifying collections:

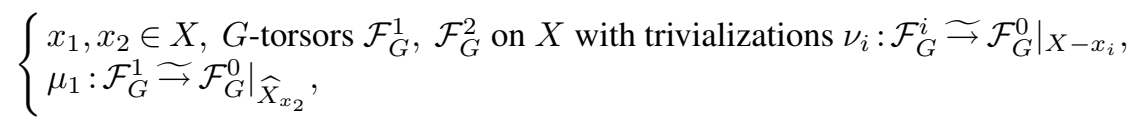

where $\widehat{X}_{x_{2}}$ is the formal neighborhood of $x_{2}$ in $X$. The map $p_{G, X}$ forgets $\mu_{1}$.

The ind-scheme $\operatorname{Conv}_{G, X}$ classifies collections:

$$
\left\{\begin{array}{l}
x_{1}, x_{2} \in X, G \text {-torsors } \mathcal{F}_{G}^{1}, \mathcal{F}_{G} \text { on } X \\
\text { isomorphisms } \nu_{1}:\left.\mathcal{F}_{G}^{1} \underset{\rightrightarrows}{\rightrightarrows} \mathcal{F}_{G}^{0}\right|_{X-x_{1}} \text {, and } \eta:\left.\mathcal{F}_{G}^{1} \underset{\rightrightarrows}{\longrightarrow} \mathcal{F}_{G}\right|_{X-x_{2}}
\end{array}\right.
$$

The map $m_{X}$ sends this collection to $\left(x_{1}, x_{2}, \mathcal{F}_{G}\right)$ together with the trivialization $\eta \circ \nu_{1}^{-1}: \mathcal{F}_{G}^{0} \widetilde{\rightrightarrows}$ $\left.\mathcal{F}_{G}\right|_{X-x_{1}-x_{2}}$.

The map $q_{G, X}$ sends (24) to the collection (25), where $\mathcal{F}_{G}$ is obtained by gluing $\mathcal{F}_{G}^{1}$ on $X-x_{2}$ and $\mathcal{F}_{G}^{2}$ on $\widehat{X}_{x_{2}}$ using their identification over $\left(X-x_{2}\right) \cap \widehat{X}_{x_{2}}$ via $\nu_{2}^{-1} \circ \mu_{1}$.

The canonical isomorphism

$$
q_{G, X}^{*} m_{X}^{*} \mathcal{L} \cong p_{G, X}^{*}(\mathcal{L} \otimes \mathcal{L})
$$

allows to define $\tilde{q}_{G, X}$ as follows. Write $M_{i}$ (respectively, $M$ ) for the vector bundle induced from $\mathcal{F}_{G}^{i}$ (respectively, $\mathcal{F}_{G}$ ) via the standard representation of $G$.

A point of $\widetilde{C}_{G, X}$ is given by (24) together with 1-dimensional vector spaces $\mathcal{B}_{1}, \mathcal{B}_{2}$ and $\mathcal{B}_{i}^{2} \underset{\rightarrow}{\rightarrow} \mathcal{L}_{\mathcal{F}_{G}^{i}}$. By Lemma 10, $\mathcal{L}_{\mathcal{F}_{G}^{i}} \underset{\rightarrow}{\rightarrow} \operatorname{det}\left(M_{0, x_{i}}: \operatorname{det} M_{i, x_{i}}\right)$.

A point of $\widetilde{\operatorname{Conv}}_{G, X}$ is given by (25) together with 1 -dimensional vector space $\mathcal{B}$ and $\mathcal{B}^{2} \underset{\sim}{\rightarrow} \mathcal{L}_{\mathcal{F}_{G}}$. We have

$$
\begin{aligned}
\mathcal{L}_{\mathcal{F}_{G}} & \simeq \frac{\operatorname{det} \mathrm{R} \Gamma\left(X, M_{0}\right)}{\operatorname{det} \mathrm{R} \Gamma\left(X, M_{1}\right)} \otimes \frac{\operatorname{det} \mathrm{R} \Gamma\left(X, M_{1}\right)}{\operatorname{det} \mathrm{R} \Gamma(X, M)} \\
& \simeq \operatorname{det}\left(M_{0, x_{1}}: M_{1, x_{1}}\right) \otimes \operatorname{det}\left(M_{1, x_{2}}: M_{x_{2}}\right) \widetilde{\mathcal{L}_{\mathcal{F}_{G}^{1}} \otimes \mathcal{L}_{\mathcal{F}_{G}^{2}},}
\end{aligned}
$$

$4^{e}$ SÉRIE - TOME $39-2006-\mathrm{N}^{\circ} 3$ 
the last isomorphism being given by $\mu_{1}: \operatorname{det}\left(M_{1, x_{2}}\right) \widetilde{\longrightarrow} \operatorname{det}\left(M_{0, x_{2}}\right)$ and $M_{x_{2}} \underset{\rightarrow}{\rightarrow} M_{2, x_{2}}$. Define $\tilde{q}_{G, X}$ by setting $\mathcal{B}=\mathcal{B}_{1} \otimes \mathcal{B}_{2}$.

As in Section 8.2 one checks that for $K_{1}, K_{2} \in \operatorname{Sph}\left(\widetilde{\mathrm{Gr}}_{G, X}\right)$ there is a (defined up to a unique isomorphism) perverse sheaf $K_{12}$ on $\widetilde{\operatorname{Conv}_{G, X}}$ with $\tilde{q}_{G, X}^{*} K_{12} \widetilde{\rightarrow} \tilde{p}_{G, X}^{*}\left(K_{1} \otimes K_{2}\right)$. Moreover, $-1 \in \mu_{2}$ acts on $K_{12}$ as -1 . We then let

$$
K_{1} *_{X} K_{2}=\tilde{m}_{X !} K_{12}
$$

Let $U \subset X^{2}$ be the complement to the diagonal. Let $j:{\widetilde{\operatorname{Gr}_{G, X}}}_{G}(U) \hookrightarrow \widetilde{\mathrm{Gr}}_{G, X^{2}}$ be the preimage of $U$. Recall that $m_{X}$ is stratified small, an isomorphism over the preimage of $U$ [20]. So, the same holds for the representable map $\tilde{m}_{X}$. Thus, $K_{1} *_{X} K_{2}$ is a perverse sheaf, the GoreskyMacPherson from $\widetilde{\mathrm{Gr}}_{G, X^{2}}(U)$. Besides, $-1 \in \mu_{2}$ acts on it as -1 . Moreover, $K_{1} *_{X} K_{2} \in$ $\operatorname{Sph}\left(\widetilde{\mathrm{Gr}}_{G, X^{2}}\right)$, because $G_{X^{2}}$-equivariance is clear over $\widetilde{\mathrm{Gr}}_{G, X^{2}}(U)$ and is preserved under the intermediate extension.

Recall the group ind-scheme $\operatorname{Aut}^{0} \mathcal{O}$ (cf. Remark 6). Let $\widehat{X} \rightarrow X$ be the $\operatorname{Aut}^{0} \mathcal{O}$-torsor whose

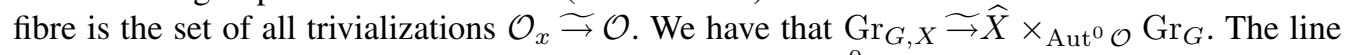
bundle $\mathcal{L}$ on $\operatorname{Gr}_{G, X}$ identifies with the descent of the $\operatorname{Aut}^{0} \mathcal{O}$-equivariant line bundle $\mathcal{O} \otimes \mathcal{L}$ under $\widehat{X} \times \operatorname{Gr}_{G} \rightarrow \operatorname{Gr}_{G, X}$. Since any $K \in \operatorname{Sph}\left(\widetilde{\operatorname{Gr}}_{G}\right)$ is $\operatorname{Aut}^{0} \mathcal{O}$-equivariant, we have a natural (fully faithful) functor

$$
\tau^{0}: \operatorname{Sph}\left(\widetilde{\mathrm{Gr}}_{G}\right) \rightarrow \operatorname{Sph}\left(\widetilde{\mathrm{Gr}}_{G, X}\right)[-1] .
$$

Let glob: $\operatorname{Sph}\left(\widetilde{\mathrm{Gr}}_{G}\right) \rightarrow \operatorname{Sph}\left(\widetilde{\mathrm{Gr}}_{G, X}\right)$ denote the functor glob $=\tau^{0}[1]$.

Now define the commutativity constraint following [20]. Let $i: \widetilde{\mathrm{Gr}}_{G, X} \rightarrow \widetilde{\mathrm{Gr}}_{G, X^{2}}$ be the preimage of the diagonal in $X^{2}$. For $F_{1}, F_{2} \in \operatorname{Sph}\left(\widetilde{\mathrm{Gr}}_{G}\right)$ letting $K_{i}=\tau^{0} F_{i}$ define

$$
\left.K_{12}\right|_{U}:=\left.K_{12}\right|_{\widetilde{\operatorname{Gr}}_{G, X^{2}}(U)}
$$

as above (but now it is placed in perverse degree 2). We get

$$
\begin{aligned}
K_{1} *_{X} K_{2} & \underset{\rightrightarrows}{\rightrightarrows} j_{!_{*}}\left(\left.K_{12}\right|_{U}\right), \\
\tau^{0}\left(F_{1} * F_{2}\right) & \stackrel{\hookrightarrow}{\rightarrow} i^{*}\left(K_{1} *_{X} K_{2}\right) .
\end{aligned}
$$

So, the involution $\sigma$ of $\widetilde{\mathrm{Gr}}_{G, X^{2}}$ interchanging $x_{i}$ yields

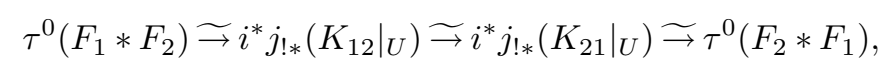

because $\left.\sigma^{*}\left(\left.K_{12}\right|_{U}\right) \widetilde{\rightrightarrows} K_{21}\right|_{U}$. (We used the functor $\tau^{0}$ instead of glob to avoid the signs ambiguity in the commutativity constraints.)

The fact that the associativity and commutativity constraints are compatible follows from a similar picture over $X^{3}$. Thus, $\operatorname{Sph}\left(\widetilde{\mathrm{Gr}}_{G}\right)$ is a tensor category.

Remark 7. - Fix $x \in X(k)$. Consider the Hecke stack ${ }_{x} \mathcal{H}_{G}$ classifying two $G$-bundles $\mathcal{F}_{G}, \mathcal{F}_{G}^{\prime}$ on $X$ together with an isomorphism $\left.\mathcal{F}_{G} \underset{\rightarrow}{\rightarrow} \mathcal{F}_{G}^{\prime}\right|_{X-x}$. Let $p$ (respectively, $p^{\prime}$ ) be the projection ${ }_{x} \mathcal{H}_{G} \rightarrow \operatorname{Bun}_{G}$ sending the above collection to $\mathcal{F}_{G}$ (respectively, $\mathcal{F}_{G}^{\prime}$ ). Write $\operatorname{Bun}_{G}^{x}$

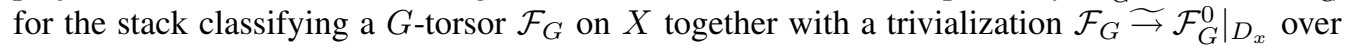
the formal disk $D_{x}$ around $x$.

Let $\gamma$ (respectively, $\gamma^{\prime}$ ) be the isomorphism $\operatorname{Bun}_{G}^{x} \times_{G\left(\mathcal{O}_{x}\right)} \operatorname{Gr}_{G, x} \widetilde{\rightrightarrows}{ }_{x} \mathcal{H}_{G}$ such that the projection to the first term corresponds to $p$ (respectively, to $p^{\prime}$ ). Write $M$ (respectively, $M^{\prime}$ ) 
for the vector bundle corresponding to $\mathcal{F}_{G}$ (respectively, to $\mathcal{F}_{G}^{\prime}$ ) via the standard representation of $G$. Write $\mathcal{L}$ for the $\left(\mathbb{Z} / 2 \mathbb{Z}\right.$-graded) line bundle on ${ }_{x} \mathcal{H}_{G}$ with fibre $\operatorname{det} \mathrm{R} \Gamma(X, M) \otimes$ $\operatorname{det} \operatorname{R} \Gamma\left(X, M^{\prime}\right)^{-1}$. Let ${ }_{x} \tilde{\mathcal{H}}_{G}$ be the gerbe of square roots of $\mathcal{L}$. Both $\gamma$ and $\gamma^{\prime}$ extend to $G\left(\mathcal{O}_{x}\right)$ torsors

$$
\tilde{\gamma}, \tilde{\gamma}^{\prime}: \operatorname{Bun}_{G}^{x} \times \widetilde{\operatorname{Gr}}_{G, x} \rightarrow{ }_{x} \widetilde{\mathcal{H}}_{G} .
$$

For $\mathcal{S} \in \operatorname{Sph}\left(\widetilde{\operatorname{Gr}}_{G, x}\right)$ denote by $\overline{\mathbb{Q}}_{\ell} \widetilde{\nabla} \mathcal{S}$ (respectively, by $\overline{\mathbb{Q}}_{\ell} \widetilde{\nabla}^{\prime} \mathcal{S}$ ) the twisted tensor product viewed as a perverse sheaf on ${ }_{x} \widetilde{\mathcal{H}}_{G}$ via $\tilde{\gamma}$ (respectively, $\left.\tilde{\gamma}^{\prime}\right)$. Given $\mathcal{S} \in \operatorname{Sph}\left(\widetilde{\operatorname{Gr}}_{G, x}\right)$ there is a (defined up to a unique isomorphism) $\mathcal{T} \in \operatorname{Sph}\left(\widetilde{\mathrm{Gr}}_{G, x}\right)$ equipped with an isomorphism $\overline{\mathbb{Q}}_{\ell} \widetilde{\otimes} \mathcal{S} \widetilde{\rightarrow} \overline{\mathbb{Q}}_{\ell} \widetilde{\nabla}^{\prime} \mathcal{T}$. This defines a covariant involution functor $\star$ on the category $\operatorname{Sph}\left(\widetilde{\operatorname{Gr}}_{G, x}\right)$ By Remark 6, we may view $\star$ as an involution functor on $\operatorname{Sph}\left(\widetilde{\mathrm{Gr}}_{G}\right)$ independently of a choice of a trivialization $\mathcal{O}_{x} \underset{\longrightarrow}{\longrightarrow}$.

In the same way as for usual spherical sheaves on $\mathrm{Gr}_{G}$ in [14], one checks that for $K_{1}, K_{2}, K_{3} \in \operatorname{Sph}\left(\widetilde{\mathrm{Gr}}_{G}\right)$ we have canonically

$$
R \operatorname{Hom}\left(K_{1} * K_{2}, K_{3}\right) \stackrel{\rightarrow}{\rightarrow} \operatorname{Hom}\left(K_{1}, K_{3} * \mathbb{D}\left(\star K_{2}\right)\right) .
$$

So, $K_{3} * \mathbb{D}\left(\star K_{2}\right)$ represents the internal $\mathcal{H o m}\left(K_{2}, K_{3}\right)$ in the sense of the tensor structure

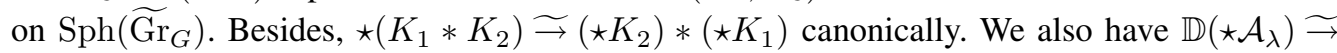
$\star \mathcal{A}_{\lambda} \widetilde{\longrightarrow} \mathcal{A}_{\lambda}$ for each $\lambda \in \Lambda_{+}$.

\subsection{Functors $F^{\theta}$}

Let $P \subset G$ denote the Siegel parabolic preserving $\mathcal{O}_{X}^{n} \subset \mathcal{O}_{X}^{n} \oplus \Omega^{n}$. Write $Q$ for the Levi quotient, so $Q \underset{\rightarrow}{\widetilde{G L}}$ canonically. Let $\check{\Lambda}_{G, P}$ denote the lattice of characters of $P /[P, P]=$ $Q /[Q, Q]$ and $\Lambda_{G, P}$ the dual lattice. Let $\check{\omega}_{n} \in \check{\Lambda}_{G, P}$ denote the fundamental weight of $G$ corresponding to the unique simple coroot which is not a coroot of $Q$. So, $\check{\omega}_{n}$ is the highest weight of an irreducible subrepresentation in $\Lambda^{n} M$, where $M$ is the standard representation of $G$. Then $\check{\omega}_{n}$ is a free generator of $\check{\Lambda}_{G, P}$.

The connected components of $\mathrm{Gr}_{Q, x}$ are indexed by $\Lambda_{G, P}$, the component $\mathrm{Gr}_{Q, x}^{\theta}$ classifies $\left(L \in \operatorname{Bun}_{n}, \nu:\left.L \widetilde{\rightarrow} \mathcal{O}^{n}\right|_{X-x}\right)$ such that $\operatorname{deg} L=-\left\langle\theta, \check{\omega}_{n}\right\rangle$. The reduced part $\operatorname{Gr}_{Q, x, \text { red }}^{\theta} \hookrightarrow \operatorname{Gr}_{Q, x}^{\theta}$ is the ind-scheme classifying $\left(L \in \operatorname{Bun}_{n}, \nu:\left.L \widetilde{\Im} \mathcal{O}^{n}\right|_{X-x}\right)$ that induce an isomorphism

$$
\operatorname{det} L \widetilde{\mathcal{O}}\left(-\left\langle\theta, \check{\omega}_{n}\right\rangle x\right) .
$$

Following [5], for $\theta \in \Lambda_{G, P}$ let $S_{P}^{\theta}$ denote the ind-scheme classifying: $\left(\mathcal{F}_{P}, \nu\right)$, where $\mathcal{F}_{P}$ is a $P$-torsor on $X$ and $\nu:\left.\mathcal{F}_{P} \underset{\rightarrow}{\rightarrow} \mathcal{F}_{P}^{0}\right|_{X-x}$ is a trivialization such that $\left(\mathcal{F}_{P} \times_{P} Q, \nu\right)$ lies in $\operatorname{Gr}_{Q, x}^{\theta}$. In other words, $S_{P}^{\theta}$ classifies a $P$-torsor given by an exact sequence $0 \rightarrow \mathrm{Sym}^{2} L \rightarrow ? \rightarrow \Omega \rightarrow 0$ on $X$ with $L \in \operatorname{Bun}_{n}$, a splitting of this sequence over $X-x$, and a trivialization $\nu:\left.L \widetilde{\rightarrow} \mathcal{O}^{n}\right|_{X-x}$ with $\operatorname{deg} L=-\left\langle\theta, \breve{\omega}_{n}\right\rangle$. The reduced part $\left(S_{P}^{\theta}\right)_{\text {red }}$ is given by the additional condition that $\nu$ induces an isomorphism (29).

We have a map $\mathfrak{s}_{P}^{\theta}: S_{P}^{\theta} \rightarrow \operatorname{Gr}_{G, x}$ sending $\left(\mathcal{F}_{P}, \nu\right)$ to $\left(\mathcal{F}_{P} \times_{P} G, \nu\right)$, its restriction $\left(S_{P}^{\theta}\right)_{\text {red }} \hookrightarrow \operatorname{Gr}_{G, x}$ is a locally closed immersion.

The map $\mathfrak{s} \frac{\theta}{P}: S \frac{\theta}{P} \rightarrow \operatorname{Gr}_{G, x}$ is defined in a similar way using the Lagrangian subbundle $\Omega^{n} \subset \mathcal{O}_{X}^{n} \oplus \Omega^{n}$ that defines the opposite parabolic subgroup $\bar{P} \subset G$.

Write $\mathfrak{t}_{P}^{\theta}: S_{P}^{\theta} \rightarrow \operatorname{Gr}_{Q, x}^{\theta}$ for the projection sending $\left(\mathcal{F}_{P}, \nu\right)$ to $\left(\mathcal{F}_{P} \times_{P} Q, \nu\right)$ and $\mathfrak{r}_{P}^{\theta}: \mathrm{Gr}_{Q, x}^{\theta} \hookrightarrow S_{P}^{\theta}$ for the natural section, similarly for $\bar{P}$.

$4^{\text {e }}$ SÉRIE - TOME $39-2006-\mathrm{N}^{\circ} 3$ 
Fix an isomorphism $\mathbb{G}_{m} \widetilde{\rightarrow} Z(Q)$, where $Z(Q)$ is the center of $Q$, in such a way that

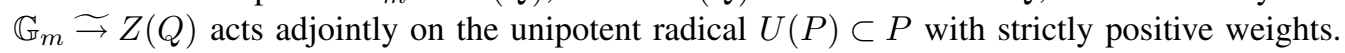
The subscheme of $Z(Q)$-fixed points in $\operatorname{Gr}_{G}$ is $Q(K) G(\mathcal{O}) / G(\mathcal{O})$, its connected components are $\mathrm{Gr}_{Q, \text { red }}^{\theta}, \theta \in \Lambda_{G, P}$. One checks that

$$
\begin{aligned}
\left\{x \in \operatorname{Gr}_{G, x} \mid \lim _{t \rightarrow 0} t x \in \operatorname{Gr}_{Q, x, \text { red }}^{\theta}\right\} & =\left(S_{P}^{\theta}\right)_{\text {red }} \quad \text { and } \\
\left\{x \in \operatorname{Gr}_{G, x} \mid \lim _{t \rightarrow \infty} t x \in \operatorname{Gr}_{Q, x, \text { red }}^{\theta}\right\} & =\left(S_{\bar{P}}^{\theta}\right)_{\text {red }} .
\end{aligned}
$$

Consider the diagram

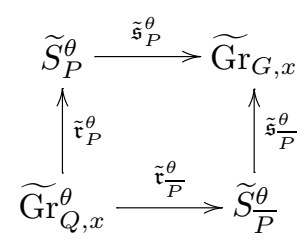

obtained by restricting the gerbe $\widetilde{\mathrm{Gr}}_{G, x} \rightarrow \mathrm{Gr}_{G, x}$ with respect to the corresponding maps.

LEMMA 11. - There exists a canonical $P\left(\mathcal{O}_{x}\right)$-equivariant section $i_{P}^{\theta}: S_{P}^{\theta} \rightarrow \widetilde{S}_{P}^{\theta}$ of the gerbe $\widetilde{S}_{P}^{\theta} \rightarrow S_{P}^{\theta}$.

Proof. - Remind the line bundle $\mathcal{L}$ on $\mathrm{Gr}_{G, x}$ introduced in 8.3. Consider the map $\operatorname{Gr}_{G, x} \rightarrow$ $\operatorname{Bun}_{G}$ sending $\left(\mathcal{F}_{G}, \nu:\left.\mathcal{F}_{G} \widetilde{\rightrightarrows} \mathcal{F}_{G}^{0}\right|_{X-x}\right)$ to $\mathcal{F}_{G}$. The restriction of $\mathcal{A}$ under this map identifies canonically with $\mathcal{L}^{-1} \otimes \operatorname{det} \mathrm{R} \Gamma\left(X, M_{0}\right)$, where $M_{0}=\mathcal{O}_{X}^{n} \oplus \Omega^{n}$. Since $\operatorname{det} \mathrm{R} \Gamma\left(X, M_{0}\right) \widetilde{\rightrightarrows}$ $\operatorname{det} \mathrm{R} \Gamma(X, \mathcal{O})^{\otimes 2 n}$, we get a Cartesian square

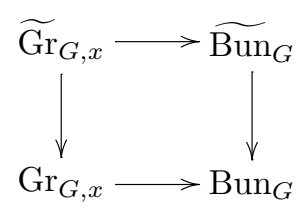

Remind the map $\tilde{\nu}$ defined in Lemma 5. Now the diagram

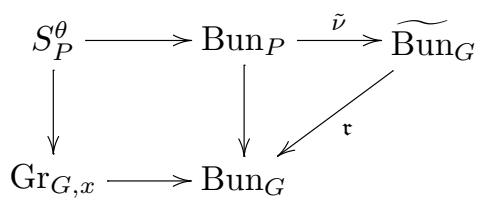

yields the section $i_{P}^{\theta}$.

To see that it is $P\left(\mathcal{O}_{x}\right)$-equivariant, rewrite it in local terms as follows. $\operatorname{On}_{\mathrm{Gr}^{\theta}, x}^{\theta}$ we have the $\mathbb{Z} / 2 \mathbb{Z}$-graded $Q\left(\mathcal{O}_{x}\right)$-equivariant line bundle, say ${ }_{\theta} \mathcal{L}$, whose fibre at $\left(L,\left.L \widetilde{\sim} \mathcal{O}^{n}\right|_{X-x}\right)$ is

$$
\operatorname{det}\left(L_{0} \otimes \mathcal{O}_{x}: L \otimes \mathcal{O}_{x}\right)
$$

with $L_{0}=\mathcal{O}_{X}^{n}$. Hence $\left(\mathfrak{t}_{P}^{\theta}\right)^{*}{ }_{\theta} \mathcal{L}$ is a $P\left(\mathcal{O}_{x}\right)$-equivariant line bundle on $S_{P}^{\theta}$. The canonical $\mathbb{Z} / 2 \mathbb{Z}$-graded $P\left(\mathcal{O}_{x}\right)$-equivariant isomorphism $\left(\mathfrak{s}_{P}^{\theta}\right)^{*} \mathcal{L} \widetilde{\longrightarrow}\left(\mathfrak{t}_{P}^{\theta}\right)^{*}\left({ }_{\theta} \mathcal{L}\right)^{\otimes 2}$ defines the section $i_{P}^{\theta}$ via 3.1.2. 
Define the functors $F^{\theta}, F^{\prime \theta}: \operatorname{Sph}\left(\widetilde{\mathrm{Gr}}_{G, x}\right) \rightarrow \mathrm{D}\left(\mathrm{Gr}_{Q, x}^{\theta}\right)$ by

$$
F^{\prime \theta}(K)=\left(\mathfrak{t}_{P}^{\theta}\right)_{!}\left(i_{P}^{\theta}\right)^{*}\left(\tilde{\mathfrak{s}}_{P}^{\theta}\right)^{*} K \quad \text { and } \quad F^{\theta}(K)=F^{\prime \theta}(K) \otimes \overline{\mathbb{Q}}_{\ell}[1]\left(\frac{1}{2}\right)^{\otimes\left\langle\theta, 2 \breve{\rho}-2 \check{\rho}_{Q}\right\rangle} .
$$

We have used the fact that $2\left(\check{\rho}-\check{\rho}_{Q}\right) \in \check{\Lambda}_{G, P}$.

Remark 8. - We could replace in the definition of $F^{\theta}$ and $F^{\prime \theta}$ the ind-schemes $S_{P}^{\theta}$ and $\mathrm{Gr}_{Q, x}^{\theta}$ by their reduced parts, the corresponding functors would be canonically isomorphic to the old ones. In some geometric questions we work rather with the corresponding reduced ind-schemes (without indicating that explicitly, for example in Propositions 12 and 15, Corollary 1 and so on).

PROPOSITION 12. - The functor $F^{\theta}(K)$ maps $\operatorname{Sph}\left(\widetilde{\mathrm{Gr}}_{G, x}\right)$ to the category $\operatorname{Sph}\left(\mathrm{Gr}_{Q, x}^{\theta}\right)$ of $Q\left(\mathcal{O}_{x}\right)$-equivariant perverse sheaves on $\mathrm{Gr}_{Q, x}^{\theta}$. In particular, it is exact.

Proof. - By Lemma 9 combined with Proposition 19, we get the hyperbolic localization functors $\operatorname{Sph}\left(\widetilde{\mathrm{Gr}}_{G, x}\right) \rightarrow \mathrm{D}\left(\widetilde{\mathrm{Gr}}_{Q, x}^{\theta}\right)$ given by

$$
K \mapsto\left(\tilde{\mathfrak{r}}_{P}^{\theta}\right)^{*}\left(\tilde{\mathfrak{s}} \frac{\theta}{P}\right)^{!} K \widetilde{\rightarrow}\left(\tilde{\mathfrak{r}}_{P}^{\theta}\right)^{!}\left(\tilde{\mathfrak{s}}_{P}^{\theta}\right)^{*} K=K^{! *}
$$

By Lemma 11 , we have moreover $K^{! *} \widetilde{\hookrightarrow}\left(\mathfrak{t}_{P}^{\theta} \times \mathrm{id}\right) !\left(\tilde{s}_{P}^{\theta}\right)^{*} K$, where

$$
\mathfrak{t}_{P}^{\theta} \times \mathrm{id}: \widetilde{S}_{P}^{\theta}=S_{P}^{\theta} \times B\left(\mu_{2}\right) \rightarrow \operatorname{Gr}_{Q, x}^{\theta} \times B\left(\mu_{2}\right)=\widetilde{\operatorname{Gr}}_{Q, x}^{\theta} .
$$

The complex $K^{! *}$ is $Q\left(\mathcal{O}_{x}\right)$-equivariant, because both $\tilde{\mathfrak{s}}_{P}^{\theta}$ and $\tilde{\mathfrak{r}}_{P}^{\theta}$ are $Q\left(\mathcal{O}_{x}\right)$-equivariant. The dimension estimates given in [5, Proposition 4.3.3] show that $F^{\theta}(K)$ is placed in nonpositive perverse degrees. Now (30) guarantees that $F^{\theta}(K)$ is placed in non-negative perverse degrees.

Let $w_{0}$ (respectively, $w_{0}^{Q}$ ) denote the longest element of the Weil group $W$ of $G$ (respectively, $W_{Q}$ of $\left.Q\right)$.

COROLlary 1.-

(i) Let $\lambda \in \Lambda^{+}$and $\theta$ be the image of $\lambda$ in $\Lambda_{G, P}$. Then $\mathcal{A}_{Q, \lambda}$ (respectively, $\mathcal{A}_{Q,-w_{0}^{Q}(\lambda)}$ ) appears with multiplicity one in $F^{\theta}\left(\mathcal{A}_{\lambda}\right)$ (respectively, in $F^{-\theta}\left(\mathcal{A}_{\lambda}\right)$ ).

(ii) The functor $F: \operatorname{Sph}\left(\widetilde{\operatorname{Gr}}_{G, x}\right) \rightarrow \operatorname{Sph}\left(\operatorname{Gr}_{Q, x}\right)$ given by $F=\bigoplus_{\theta \in \Lambda_{G, P}} F^{\theta}$ is exact and faithful.

Proof. - (i) Note that $S_{P}^{\theta} \cap \operatorname{Gr}_{G}^{\lambda}$ is open in $\operatorname{Gr}_{G}^{\lambda}$. Moreover, $\operatorname{Gr}_{Q}^{\theta} \cap \operatorname{Gr}_{G}^{\lambda}=\operatorname{Gr}_{Q}^{\lambda}$. Since $P / Q$ is affine, $\operatorname{Gr}_{Q} \hookrightarrow S_{P}$ is a closed immersion. So, $\operatorname{Gr}_{Q}^{\theta} \cap \operatorname{Gr}_{G}^{\lambda} \hookrightarrow S_{P}^{\theta} \cap \operatorname{Gr}_{G}^{\lambda}$ is a smooth closed subscheme. It follows that $\left(\tilde{\mathfrak{r}}_{P}^{\theta}\right)^{!}\left(\tilde{\mathfrak{s}}_{P}^{\theta}\right)^{*} \mathcal{A}_{\lambda}$ is a shifted constant sheaf over $\operatorname{Gr}_{Q}^{\lambda}$. The first assertion follows.

For the second, note that $\mathrm{Gr}_{Q}^{-\theta} \cap \mathrm{Gr}_{G}^{\lambda}=\mathrm{Gr}_{Q}^{-w_{0}^{Q}(\lambda)}$, and the map

$$
\mathfrak{t}_{P}^{-\theta}: S_{P}^{-\theta} \cap \mathrm{Gr}_{G}^{\lambda} \rightarrow \mathrm{Gr}_{Q}^{-\theta}
$$

is an isomorphism over the $Q(\mathcal{O})$-orbit $\mathrm{Gr}_{Q}^{-w_{0}^{Q}(\lambda)}$.

(ii) Since $F$ is exact, to show faithfulness, it suffices to prove that $F$ does not annihilate a nonzero object. To this end, it suffices to show that $F\left(\mathcal{A}_{\lambda}\right) \neq 0$ for any dominant coweight $\lambda$, which follows from (i).

4 SÉRIE - TOME $39-2006-\mathrm{N}^{\circ} 3$ 


\subsection{Example: explicit calculation}

Let $\alpha \in \Lambda^{+}$denote the coroot of $\mathbb{S p}_{2 n}$ corresponding to the maximal root $\check{\alpha}_{\max }$ of $\mathbb{S p}_{2 n}$. So, $\alpha$ is the highest weight of the standard representation of the Langlands dual group $\mathrm{SO}_{2 n+1}$ of $\mathrm{Sp}_{2 n}$. For this subsection take $G$ to be that of 8.1 for $M_{0}=\mathcal{O}^{n} \oplus \Omega_{\mathcal{O}}^{n}$. The closure $\overline{G r}_{G}^{\alpha}$ of $\operatorname{Gr}_{G}^{\alpha}$ in $\mathrm{Gr}_{G}$ is described as follows.

The $G(k)$-orbit $V$ in $\operatorname{Gr}_{G}$ passing through $\alpha(t) G(\mathcal{O})$ is identified with the projective space $V \widetilde{\longrightarrow} \mathbb{P}^{2 n-1}$, and $\mathrm{Gr}_{G}^{\alpha}$ is the total space of the line bundle $\mathcal{O}(2)$ over $V$.

Let $V=\mathbb{P}^{2 n-1} \hookrightarrow \mathbb{P}^{n(2 n+1)-1}$ be the Veronese map. Write $x_{1}, \ldots, x_{2 n}$ for the homogeneous coordinates in $\mathbb{P}^{2 n-1}$ and $t_{i j}$ with $1 \leqslant i \leqslant j \leqslant 2 n$ for the homogeneous coordinates in $\mathbb{P}^{n(2 n+1)-1}$. Then the inclusion is given by $t_{i j}=x_{i} x_{j}$. Its image is the subscheme defined by homogeneous equations

$$
t_{i j} t_{k l}=t_{i k} t_{j l}
$$

for all $i, j, k, l$ whenever this makes sense.

One may identify the Lie algebra of $\mathbb{S p}_{2 n}$ with $\mathbb{A}^{n(2 n+1)}$ in such a way that the set $Z$ of elements $\mathbb{S p}_{2 n}$-conjugate to a multiple of the maximal root becomes the subscheme $Z \subset \mathbb{A}^{n(2 n+1)}=\operatorname{Spec} k\left[t_{i j}\right]$ given by Eqs. (31). Let $A \in Z$ denote the origin of this cone. Let $\bar{Z} \subset \mathbb{P}^{n(2 n+1)}$ be the projective closure of $Z$. Then $\overline{\operatorname{Gr}}_{G}^{\alpha}=\bar{Z}$ and $\operatorname{Gr}_{G}^{\alpha}=\bar{Z}-A$.

The projection $\pi: \bar{Z}-A \rightarrow V$ is an affine fibration on which $\mathcal{O}(2)$ acts transitively and freely (and the corresponding torsor is trivial). So, $\pi^{*}$ yields a diagram of isomorphisms

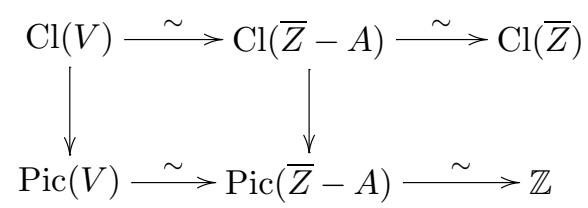

where for a variety $S$ we denote by $\mathrm{Cl}(S)$ the Weil divisors class group.

Write $\left(t_{i j}, w\right)$ for the homogeneous coordinates in $\mathbb{P}^{n(2 n+1)}$. Let the subscheme $V \subset \bar{Z}$ be given by $w=0$, it is a section of $\pi$. We have $Z=\bar{Z}-V$.

The image in $\mathrm{Cl}(V)$ of the hyperplane section of $\mathbb{P}^{n(2 n+1)-1}$ is 2 . It follows that the image of $V$ in $\mathrm{Cl}(\bar{Z})$ is 2 and $\mathrm{Cl}(Z) \widetilde{Z} / 2 \mathbb{Z}$.

Let $L \subset Z$ denote the preimage under $\pi$ of the subscheme of $V$ given by $x_{1}=0$. Denote again by $L$ the corresponding Weil divisor on $\bar{Z}$. Then $L$ is not locally principal in $\mathcal{O}_{Z, A}$. Indeed, let $\mathfrak{p} \subset \mathcal{O}_{Z, A}$ denote the ideal corresponding to $L$ and $\mathfrak{m}_{Z, A} \subset \mathcal{O}_{Z, A}$ the maximal ideal. Then $t_{i j}(1 \leqslant i \leqslant j \leqslant n)$ form a base in the cotangent space $\mathfrak{m}_{Z, A} / \mathfrak{m}_{Z, A}^{2}$, and the elements $t_{1 j} \in \mathfrak{p}$ $(1 \leqslant j \leqslant n)$ are linearly independent in $\mathfrak{m}_{Z, A} / \mathfrak{m}_{Z, A}^{2}$. So, Pic $Z=0$, and $\mathcal{O}_{\bar{Z}}(V)$ generates $\operatorname{Pic}(\bar{Z})$. The image of $\mathcal{O}_{\bar{Z}}(V)$ under the composition

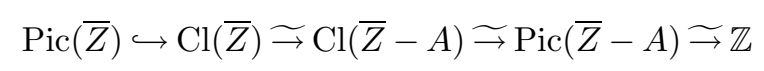

is 2 . In other words, $\mathcal{O}_{\bar{Z}-A}(L)$ does not extend to a line bundle on $\bar{Z}$.

The line bundle $\left.\mathcal{L}\right|_{\overline{G r}_{G}^{\alpha}} ^{\alpha}$ identifies with $\left.\mathcal{O}_{\mathbb{P}^{n(2 n+1)}}(1)\right|_{\bar{Z}}$. Let $\widetilde{Z} \rightarrow \bar{Z}$ denote the $\mu_{2}$-gerbe of square roots of this bundle. We see that this gerbe is nontrivial, though trivial over $\bar{Z}-A$.

Set $Y=\mathbb{A}^{2 n}=\operatorname{Spec} k\left[x_{i}\right]$. Let $\tau: Y \rightarrow Z$ be the map given by $t_{i j}=x_{i} x_{j}$. Clearly, $Y-\tau^{-1}(A) \rightarrow Z-A$ is a $S_{2}$-Galois covering.

For a coweight $\lambda$ of $Q$ denote by $\mathcal{A}_{Q, \lambda}$ the intersection cohomology sheaf of the $Q(\mathcal{O})$-orbit on $\operatorname{Gr}_{Q}$ passing through $\lambda(t) Q(\mathcal{O})$. 
PROPOSITION 13.-

(1) The sheaf $\mathcal{A}_{\alpha}$ is the extension by zero from $\bar{Z}-A$.

(2) We have $F^{0}\left(\mathcal{A}_{\alpha}\right)=0$. For $\theta \in \Lambda_{G, P}$ such that $\left\langle\theta, \check{\omega}_{n}\right\rangle=1$ we have $F^{\theta}\left(\mathcal{A}_{\alpha}\right) \widetilde{\rightarrow} \mathcal{A}_{Q, \alpha}$ and $F^{-\theta}\left(\mathcal{A}_{\alpha}\right) \stackrel{\sim}{\rightarrow} \mathcal{A}_{Q,-\alpha}$.

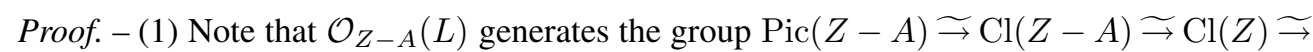
$\mathbb{Z} / 2 \mathbb{Z}$. The gerbe $\widetilde{Z}$ is obtained by gluing together trivial gerbes $Z \times B\left(\mu_{2}\right)$ and $(\bar{Z}-A) \times B\left(\mu_{2}\right)$ over $Z-A$. The gluing data is an automorphism of the gerbe $(Z-A) \times B\left(\mu_{2}\right)$ which can be described as follows.

An $S$-point of $(Z-A) \times B\left(\mu_{2}\right)$ is a line bundle $\mathcal{B}$ on $S$ together with $\mathcal{B}^{2} \widetilde{\mathcal{O}_{S}}$ and a map $S \rightarrow(Z-A)$. Our automorphism sends this point to the same map $S \rightarrow(Z-A)$ and replaces $\mathcal{B}$ by $\mathcal{B}$ tensored with the restriction of $\mathcal{O}_{Z-A}(L)$ to $S$.

We have the $\mu_{2}$-torsor over $Z-A$ consisting of those sections of $\mathcal{O}_{Z-A}(L)$ whose square is 1 . This is exactly the Galois covering $Y-\tau^{-1}(A) \rightarrow Z-A$.

Let $W$ denote the nontrivial rank one local system on $B\left(\mu_{2}\right)$ corresponding to the covering Spec $k \rightarrow B\left(\mu_{2}\right)$. If we identify our gerbe over $Z$ with $Z \times B\left(\mu_{2}\right)$ then over that locus $\mathcal{A}_{\alpha}$ becomes the exterior product $N \otimes W$, where $N$ is the nontrivial local system on $Z-A$ extended by zero to $A$ and corresponding to the covering $Y-\tau^{-1}(A) \rightarrow Z-A$.

(2) Considering $\mathrm{Gr}_{Q}^{0}$ as a subscheme of $\mathrm{Gr}_{G}$, one checks that $\mathrm{Gr}_{Q}^{0} \cap \overline{\mathrm{Gr}}_{G}^{\alpha}$ is the point scheme $1 \in \operatorname{Gr}_{G}$. Consider the $*$-restriction $\left.N\right|_{Z \cap L}$. Since the !-fibre at $A$ of $\left.N\right|_{Z \cap L}$ vanishes, we get $F^{0}\left(\mathcal{A}_{\alpha}\right)=0$.

Let $\theta \in \Lambda_{G, P}$ be such that $\left\langle\theta, \check{\omega}_{n}\right\rangle=1$. Recall the map $\pi: \bar{Z}-A \rightarrow V$. We have

$$
\operatorname{Gr}_{G}^{\alpha} \cap S_{P}^{\theta}=\pi^{-1}\left(V_{0}\right),
$$

where $V_{0} \subset V=\mathbb{P}\left(M_{0}(x) / M_{0}\right)$ is the complement to $\mathbb{P}\left(L_{0}(x) / L_{0}\right)$. In other words, $\mathrm{Gr}_{G}^{\alpha} \cap S_{P}^{\theta} \subset \mathrm{Gr}_{G}^{\alpha}$ is the open subscheme given by the condition that the line $\left(M+M_{0}\right) / M_{0}$ is not contained in $L_{0}(x) / L_{0}$. Further, $\operatorname{Gr}_{G}^{\alpha} \cap \operatorname{Gr}_{Q}^{\theta}=\operatorname{Gr}_{Q}^{\alpha}$. The isomorphism $F^{\theta}\left(\mathcal{A}_{\alpha}\right) \stackrel{\sim}{\rightarrow} \mathcal{A}_{Q, \alpha}$ follows.

We have $\operatorname{Gr}_{G}^{\alpha} \cap S_{P}^{-\theta}=\operatorname{Gr}_{Q}^{-\alpha}$. This yields the last isomorphism.

Remark 9. - Let $\lambda \in \Lambda^{+}$and $\theta \in \Lambda_{G, P}$. If $F^{\theta}\left(\mathcal{A}_{\lambda}\right) \neq 0$ then

$$
-\left\langle\lambda, \check{\omega}_{n}\right\rangle \leqslant\left\langle\theta, \check{\omega}_{n}\right\rangle \leqslant\left\langle\lambda, \check{\omega}_{n}\right\rangle .
$$

Indeed, if $S_{P}^{\theta} \cap \overline{\mathrm{Gr}}_{G}^{\lambda} \neq \emptyset$ then (32) holds. More generally, for a reductive group $G$ and its parabolic subgroup $P$ the condition $S_{P}^{\theta} \cap \overline{\operatorname{Gr}}_{G}^{\lambda} \neq \emptyset$ implies $\left\langle\lambda, w_{0}(\check{\lambda})\right\rangle \leqslant\langle\theta, \check{\lambda}\rangle \leqslant\langle\lambda, \check{\lambda}\rangle$ for any $\check{\lambda} \in \check{\Lambda}_{G, P}$ which is dominant for $G$.

\subsection{The functors $F_{X^{d}}^{\theta}$}

Let $\operatorname{Gr}_{Q, X^{d}}$ denote the ind-scheme classifying $\left(x_{1}, \ldots, x_{d}\right) \in X^{d}$ and $L \in \operatorname{Bun}_{n}$ with trivialization $\left.L \widetilde{\rightarrow} \mathcal{O}^{n}\right|_{X-x_{1} \cup \cdots \cup x_{d}}$. Its connected components are indexed by $\Lambda_{G, P}$, the component $\operatorname{Gr}_{Q, X^{d}}^{\theta}$ is given by $\operatorname{deg} L=-\left\langle\theta, \check{\omega}_{n}\right\rangle$. We have a natural map $\operatorname{Gr}_{Q, X^{d}} \rightarrow \operatorname{Gr}_{G, X^{d}}$ sending the above point to $L \oplus\left(L^{*} \otimes \Omega\right)$ with the induced trivialization outside $x_{i}$. The composition

$$
\left(\operatorname{Gr}_{Q, X^{d}}\right)_{\mathrm{red}} \hookrightarrow \mathrm{Gr}_{Q, X^{d}} \rightarrow \mathrm{Gr}_{G, X^{d}}
$$

is a closed immersion.

$4^{\text {e }}$ SÉRIE - TOME $39-2006-\mathrm{N}^{\circ} 3$ 
For $\theta \in \Lambda_{G, P}$ denote by $S_{P, X^{d}}^{\theta}$ the ind-scheme classifying collections: $\left(x_{1}, \ldots, x_{d}\right) \in X^{d}$, a $P$-torsor $\mathcal{F}_{P}$ on $X$ with trivialization $\nu:\left.\mathcal{F}_{P} \widetilde{\rightarrow} \mathcal{F}_{P}^{0}\right|_{X-x_{1} \cup \ldots \cup x_{d}}$ such that the induced $Q$-torsor $\mathcal{F}_{P} \times_{P} Q$ lies in $\operatorname{Gr}_{Q, X^{d}}^{\theta}$. Here $\mathcal{F}_{P}^{0}$ is the $G$-torsor $\mathcal{F}_{G}^{0}=\mathcal{O}_{X}^{n} \oplus \Omega^{n}$ with $P$-structure corresponding to the Lagrangian subbundle $\mathcal{O}_{X}^{n}$.

Considering $\mathcal{F}_{\bar{P}}^{0}$ as $\mathcal{F}_{G}^{0}$ with $\bar{P}$-structure given by $\Omega^{n}$, one similarly defines the ind-scheme $S \frac{\theta}{P, X^{d}}$. As in 8.4, one defines a diagram

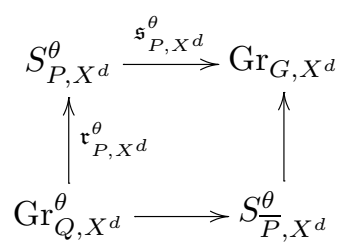

Both $\left(S_{P, X^{d}}^{\theta}\right)_{\text {red }}$ and $\left(S_{\bar{P}, X^{d}}^{\theta}\right)_{\text {red }}$ are locally closed in $\mathrm{Gr}_{G, X^{d}}$, and their intersection is $\left(\mathrm{Gr}_{Q, X^{d}}^{\theta}\right)_{\text {red }}$.

For a $k$-point $\left(x_{1}, \ldots, x_{d}\right) \in X^{d}$ with $\left\{x_{1}, \ldots, x_{d}\right\}=\left\{y_{1}, \ldots, y_{s}\right\}$ and $y_{i}$ pairwise distinct, the fibre of the diagram (33) over $\left(x_{1}, \ldots, x_{d}\right) \in X^{d}$ is

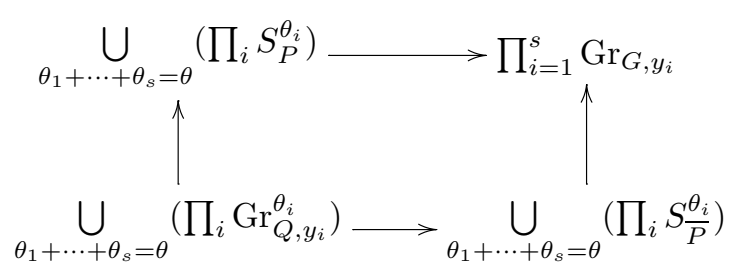

Similarly to $G_{X^{d}}$, one defines a group scheme $Q_{X^{d}}$ (respectively, $P_{X^{d}}$ ) over $X^{d}$, it acts naturally on $\operatorname{Gr}_{Q, X^{d}}^{\theta}$ (respectively, on $S_{P, X^{d}}^{\theta}$ ). Denote by $\operatorname{Sph}\left(\operatorname{Gr}_{Q, X^{d}}^{\theta}\right)$ the category of $Q_{X^{d}}$-equivariant perverse sheaves on $\operatorname{Gr}_{Q, X^{d}}^{\theta}$. Let us define the functors

$$
F_{X^{d}}^{\theta}, F_{X^{d}}^{\prime \theta}: \operatorname{Sph}\left(\widetilde{\operatorname{Gr}}_{G, X^{d}}\right) \rightarrow \mathrm{D}\left(\operatorname{Gr}_{Q, X^{d}}^{\theta}\right) .
$$

Let $\tilde{\mathfrak{s}}_{P, X^{d}}^{\theta}: \widetilde{S}_{P, X^{d}}^{\theta} \rightarrow \widetilde{\mathrm{Gr}}_{G, X^{d}}$ be the map obtained by the base change $\widetilde{\mathrm{Gr}}_{G, X^{d}} \rightarrow \operatorname{Gr}_{G, X^{d}}$ from (33). As in Lemma 11, one defines a $P_{X^{d}}$-equivariant section $i_{P, X^{d}}^{\theta}: S_{P, X^{d}}^{\theta} \rightarrow \widetilde{S}_{P, X^{d}}^{\theta}$ of the gerbe $\widetilde{S}_{P, X^{d}}^{\theta} \rightarrow S_{P, X^{d}}^{\theta}$. We have a $Q_{X^{d}}$-equivariant line bundle ${ }_{\theta} \mathcal{L}_{X^{d}}$ on $\operatorname{Gr}_{Q, X^{d}}^{\theta}$, whose fibre at

$$
\left(L,\left.L \widetilde{\mathcal{O}^{n}}\right|_{X-x_{1} \cup \cdots \cup x_{d}}\right)
$$

is $\operatorname{det} \mathrm{R} \Gamma\left(X, \mathcal{O}_{X}^{n}\right) \otimes \operatorname{det} \mathrm{R} \Gamma(X, L)^{-1}$. As $\mathbb{Z} / 2 \mathbb{Z}$-graded, it is placed in degree $b(\theta):=$ $\left\langle\theta, \breve{\omega}_{n}\right\rangle \bmod 2$. The canonical $P_{X^{d}}$-equivariant $\mathbb{Z} / 2 \mathbb{Z}$-graded isomorphism

$$
\left.\left(\mathfrak{s}_{P, X^{d}}^{\theta}\right)^{*} \mathcal{L} \widetilde{\rightarrow} \mathcal{L}_{X^{d}}^{\otimes 2}\right|_{S_{P, X^{d}}^{\theta}}
$$

yields $i_{P, X^{d}}^{\theta}$ via 3.1.2. Set

$$
F_{X^{d}}^{\prime \theta}(K)=\left(\mathfrak{r}_{P, X^{d}}^{\theta}\right)^{!}\left(i_{P, X^{d}}^{\theta}\right)^{*}\left(\tilde{\mathfrak{s}}_{P, X^{d}}^{\theta}\right)^{*} K \quad \text { and }
$$




$$
F_{X^{d}}^{\theta}(K)=F_{X^{d}}^{\prime \theta}(K) \otimes \overline{\mathbb{Q}}_{\ell}[1]\left(\frac{1}{2}\right)^{\otimes\left\langle\theta, 2 \check{\rho}-2 \check{\rho}_{Q}\right\rangle} .
$$

Note that

$$
F_{X^{d}}^{\prime \theta}(K) \widetilde{\rightrightarrows}\left(\mathfrak{t}_{P, X^{d}}^{\theta}\right)_{!}\left(i_{P, X^{d}}^{\theta}\right)^{*}\left(\widetilde{\mathfrak{s}}_{P, X^{d}}^{\theta}\right)^{*} K
$$

where $\mathfrak{t}_{P, X^{d}}^{\theta}: S_{P, X^{d}}^{\theta} \rightarrow \operatorname{Gr}_{Q, X^{d}}^{\theta}$ is the corresponding contraction map.

Remind the definition of the tensor category $\operatorname{Sph}\left(\mathrm{Gr}_{Q, x}\right)^{\natural}$. Equip $\operatorname{Sph}\left(\mathrm{Gr}_{Q, x}\right)$ with the convolution product, associativity and commutativity constraints given by the fusion procedure, then $\operatorname{Sph}\left(\mathrm{Gr}_{Q, x}\right)$ is a tensor category [20]. It has a canonical $\mathbb{Z} / 2 \mathbb{Z}$-grading compatible with the tensor structure, namely $\mathcal{A}_{Q, \lambda}$ is even (respectively, odd) if $\operatorname{dim} \operatorname{Gr}_{Q}^{\lambda}$ is even (respectively, odd). The latter condition depends only on the connected component of $\mathrm{Gr}_{Q, x}$ containing $\mathrm{Gr}_{Q, x}^{\lambda}$.

Following [20, Section 6.2], we define $\operatorname{Sph}\left(\mathrm{Gr}_{Q, x}\right)^{\natural}$ as the full subcategory of even objects in $\operatorname{Sph}\left(\operatorname{Gr}_{Q, x}\right) \otimes \operatorname{Vect}^{\varepsilon}$. We have an equivalence of monoidal categories $\operatorname{Sph}\left(\operatorname{Gr}_{Q, x}\right)^{\natural} \rightarrow$ $\operatorname{Sph}\left(\operatorname{Gr}_{Q, x}\right)$ (i.e., it is compatible with tensor product and associativity constraint, and the commutativity constraints $A \otimes B \widetilde{\rightarrow} B \otimes A$ in these two categories differ by $(-1)^{\operatorname{deg} A \operatorname{deg} B}$ ).

Let $h^{\varepsilon}: \operatorname{Sph}\left(\operatorname{Gr}_{Q, x}\right) \rightarrow \operatorname{Vect}^{\varepsilon}$ denote the global cohomology functor. Since $h^{\varepsilon}$ is a tensor functor compatible with $\mathbb{Z} / 2 \mathbb{Z}$-gradings, it gives rise to a tensor functor

$$
h: \operatorname{Sph}\left(\operatorname{Gr}_{Q, x}\right)^{\natural} \rightarrow \text { Vect. }
$$

By [20], $h$ is a fibre functor, and there is an isomorphism Aut $^{\otimes} h \widetilde{\rightarrow} \check{Q}$, where $\check{Q}$ is the Langlands dual group to $Q$ (in [20,14] some properties of the action of $\check{Q}$ on $h$ are listed, which

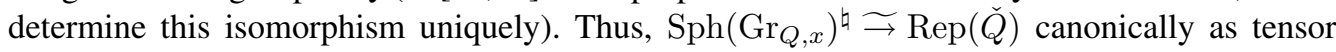
categories.

Consider

$$
\operatorname{Sph}^{\prime}\left(\operatorname{Gr}_{Q, x}\right):=\bigoplus_{\theta \in \Lambda_{G, P}} \operatorname{Sph}\left(\operatorname{Gr}_{Q, x}^{\theta}\right)\left[\left\langle\theta, 2 \check{\rho}_{Q}-2 \check{\rho}\right\rangle\right] \subset \mathrm{D}\left(\operatorname{Gr}_{Q, x}\right)
$$

equipped with the convolution product, commutativity and associativity constraints given by the fusion procedure, $\operatorname{so} \operatorname{Sph}^{\prime}\left(\mathrm{Gr}_{Q, x}\right)$ is a tensor category.

LEMMA 12. - There is a canonical equivalence of tensor categories

$$
\operatorname{Sph}^{\prime}\left(\operatorname{Gr}_{Q}\right) \stackrel{\rightarrow}{\rightarrow} \operatorname{Sph}\left(\operatorname{Gr}_{Q}\right)^{\natural}
$$

Proof. - Note that $2\left(\check{\rho}-\check{\rho}_{Q}\right)=(n+1) \check{\omega}_{n} \in \check{\Lambda}_{G, P}$. Consider the case of $n$ odd. In this case

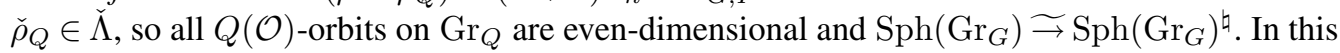
case the shifts in (34) are even, and we are done.

Consider the case of $n$ even. The component $\operatorname{Gr}_{Q, x}^{\theta}$ is even iff $\left\langle\theta, \check{\omega}_{n}\right\rangle$ is even. So, in (34) the even (respectively, odd) objects of $\operatorname{Sph}\left(\mathrm{Gr}_{Q, x}\right)$ are shifted by even (respectively, odd) cohomological degree. Our assertion follows.

Equip $\operatorname{Sph}^{\prime}\left(\operatorname{Gr}_{Q, x}\right)$ with a new $\mathbb{Z} / 2 \mathbb{Z}$-grading such that $K \in \operatorname{Sph}^{\prime}\left(\operatorname{Gr}_{Q, x}^{\theta}\right)$ is placed in degree $b(\theta)$. This $\mathbb{Z} / 2 \mathbb{Z}$-grading is compatible with the tensor structure. Denote by $\operatorname{Sph}^{\prime}\left(\operatorname{Gr}_{Q, x}\right)^{b}$ the category of even objects in $\operatorname{Sph}^{\prime}\left(\operatorname{Gr}_{Q, x}\right) \otimes \operatorname{Vect}^{\varepsilon}$, it is equipped with the induced $\mathbb{Z} / 2 \mathbb{Z}$-grading.

The proof of part (ii) of the following proposition is postponed to Section 8.7.

$4^{\text {e }}$ SÉRIE - TOME $39-2006-\mathrm{N}^{\circ} 3$ 
PROPOSITION 14. -

(i) The functor $F^{\prime}: \operatorname{Sph}\left(\widetilde{\operatorname{Gr}}_{G, x}\right) \rightarrow \operatorname{Sph}^{\prime}\left(\operatorname{Gr}_{Q, x}\right)^{b}$ given by $F^{\prime}=\bigoplus_{\theta \in \Lambda_{G, P}} F^{\prime \theta}$ is a tensor functor.

(ii) There is a unique $\mathbb{Z} / 2 \mathbb{Z}$-grading on $\operatorname{Sph}\left(\widetilde{\mathrm{Gr}}_{G, x}\right)$ such that $F^{\prime}$ is compatible with $\mathbb{Z} / 2 \mathbb{Z}$-gradings.

Proof. - (i) Pick $F_{1}, F_{2} \in \operatorname{Sph}\left(\widetilde{\mathrm{Gr}}_{G}\right)$. Set $K_{i}=\tau^{0} F_{i}$,

$$
K=F_{X^{2}}^{\theta}\left(K_{1} *_{X} K_{2}\right) \quad \text { and } \quad K^{\prime}=F_{X^{2}}^{\prime \theta}\left(K_{1} *_{X} K_{2}\right),
$$

where $\tau^{0}$ is given by (26). By abuse of notation, write also $\tau^{0}: \operatorname{Sph}\left(\operatorname{Gr}_{Q}\right) \rightarrow \operatorname{Sph}\left(\operatorname{Gr}_{Q, X}\right)[-1]$ for the corresponding functor for $Q$.

Step 1. Recall that $U \subset X^{2}$ denotes the complement to the diagonal. Write $\widetilde{\mathrm{Gr}}_{G, X^{2}}(U)$ for the

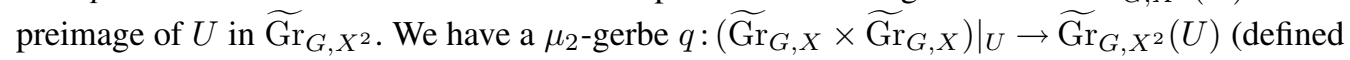
as the map $\tilde{q}_{G, X}$ in 8.3.1). The complex $q^{*}\left(K_{1} *_{X} K_{2}\right)$ identifies canonically with $\left.\left(K_{1} \otimes K_{2}\right)\right|_{U}$. Denote by $i^{\theta}$ the composition

$$
S_{P, X^{d}}^{\theta} \stackrel{i_{P, X^{d}}^{\theta}}{\longrightarrow} \widetilde{S}_{P, X^{d}}^{\theta} \stackrel{\tilde{\mathfrak{s}}_{P, X^{d}}^{\theta}}{\longrightarrow} \widetilde{\mathrm{Gr}}_{G, X^{d}}
$$

For $\theta_{1}+\theta_{2}=\theta$ the following diagram is 2 -commutative

$$
\begin{aligned}
& \left.\left(\widetilde{\mathrm{Gr}}_{G, X} \times \widetilde{\mathrm{Gr}}_{G, X}\right)\right|_{U} \stackrel{q}{\longrightarrow} \widetilde{\operatorname{Gr}}_{G, X^{2}}(U) \\
& \uparrow i^{\theta_{1} \times i^{\theta_{2}}} \quad \uparrow i^{\theta} \\
& \left.\left(S_{P, X}^{\theta_{1}} \times S_{P, X}^{\theta_{2}}\right)\right|_{U} \longrightarrow S_{P, X^{2}}^{\theta}(U)
\end{aligned}
$$

where the low horizontal arrow is the natural open immersion. However, the 2-morphism rending this diagram 2-commutative is well-defined only up to a sign, we normalize it as follows.

Write ${ }_{\theta} \underline{\mathcal{L}}_{X^{d}}$ for the line bundle ${ }_{\theta} \mathcal{L}_{X^{d}}$ viewed as ungraded. It suffices to pick an isomorphism

$$
\varepsilon^{\theta_{1}, \theta_{2}}:{ }_{\theta_{1}} \underline{\mathcal{L}}_{X} \nabla_{\theta_{2}} \underline{\mathcal{L}}_{X} \widetilde{\rightarrow}\left(j^{\theta_{1}, \theta_{2}}\right)^{*}{ }_{\theta} \underline{\mathcal{L}}_{X^{2}}
$$

where $j^{\theta_{1}, \theta_{2}}:\left.\left(\operatorname{Gr}_{Q, X}^{\theta_{1}} \times \operatorname{Gr}_{Q, X}^{\theta_{2}}\right)\right|_{U} \hookrightarrow \operatorname{Gr}_{Q, X^{2}}^{\theta}(U)$ is the natural open immersion. The order of points in $X^{2}$ yields such $\varepsilon^{\theta_{1}, \theta_{2}}$, and the usual Leibnitz rule is satisfied.

Namely, remind that $\sigma$ denotes the involution of $X^{2}$ permuting the points. For the diagram

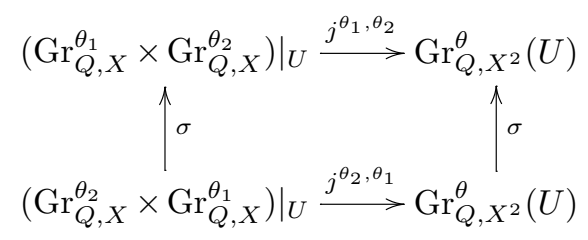


the following diagram commutes

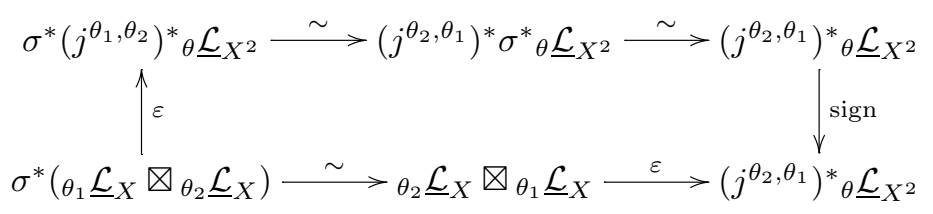

where sign $=(-1)^{b\left(\theta_{1}\right) b\left(\theta_{2}\right)}$, and the isomorphisms denoted by $\widetilde{\longrightarrow}$ are the canonical ones.

Step 2. Note that $\operatorname{Gr}_{Q, X^{2}}^{\theta}(U)$ is the disjoint union of $\left.\left(\operatorname{Gr}_{Q, X}^{\theta_{1}} \times \operatorname{Gr}_{Q, X}^{\theta_{2}}\right)\right|_{U}$ for $\theta_{1}+\theta_{2}=\theta$. Let us show that $K[2]$ is a perverse sheaf on $\operatorname{Gr}_{Q, X^{2}}^{\theta}$, the Goresky-MacPherson extension from $\operatorname{Gr}_{Q, X^{2}}^{\theta}(U)$. More precisely, we show that $\varepsilon$ as above yields an isomorphism

$$
\left(\tau^{0} F^{\prime}\left(F_{1}\right)\right) *_{X}\left(\tau^{0} F^{\prime}\left(F_{2}\right)\right) \stackrel{\hookrightarrow}{\rightarrow} F_{X^{2}}^{\prime}\left(K_{1} *_{X} K_{2}\right) .
$$

Indeed, $\varepsilon^{\theta_{1}, \theta_{2}}$ yields an isomorphism between the restriction of $K^{\prime}$ to $\left.\left(\operatorname{Gr}_{Q, X}^{\theta_{1}} \times \operatorname{Gr}_{Q, X}^{\theta_{2}}\right)\right|_{U}$ and

$$
\tau^{0} F^{\prime \theta_{1}}\left(F_{1}\right) \otimes \tau^{0} F^{\prime \theta_{2}}\left(F_{2}\right) .
$$

So, $K[2]$ is a perverse sheaf over $\operatorname{Gr}_{Q, X^{2}}^{\theta}(U)$. Using (28), we learn that the $*$-restriction of $K$ under the diagonal embedding $\operatorname{Gr}_{Q, X} \hookrightarrow \operatorname{Gr}_{Q, X^{2}}$ identifies with $\tau^{0} F^{\theta}\left(F_{1} * F_{2}\right)$, so it is placed in perverse degree 1. Now argue as in Proposition 12, using the corresponding $\mathbb{G}_{m}$-action on $\widetilde{\mathrm{Gr}}_{G, X^{2}}$. By Proposition 19, the !-restriction of $K$ under $\mathrm{Gr}_{Q, X} \hookrightarrow \mathrm{Gr}_{Q, X^{2}}$ is placed in perverse degree 3. We have constructed the isomorphism (36).

Restricting to the diagonal, it yields $\tau^{0}\left(F^{\prime}\left(F_{1}\right) * F^{\prime}\left(F_{2}\right)\right) \widetilde{\rightarrow} \tau^{0} F^{\prime}\left(F_{1} * F_{2}\right)$.

Step 3 . Let us check the compatibility with the commutativity constraints. Using (35) one shows that the diagram commutes

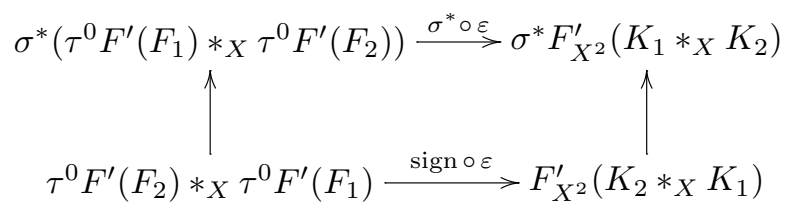

where the vertical arrows are the canonical isomorphisms, and sign is that from step 1 . We are done.

\subsection{The structure of $\operatorname{Sph}\left(\widetilde{\mathrm{Gr}}_{G}\right)$}

Recall that $\Lambda_{G, P}$ is canonically identified with the lattice of characters of the center $Z(\check{Q})$ of the Langlands dual group $\check{Q}$ of $Q$. For a representation $V$ of $\mathrm{SO}_{2 n+1}$ and $\theta \in \Lambda_{G, P}$ write $V_{\theta}$ for the direct summand of $V$ on which $Z(\check{Q})$ acts by $\theta$.

For $\lambda \in \Lambda^{+}$write $V^{\lambda}$ for the irreducible representation of $\mathrm{SO}_{2 n+1}$ of highest weight $\lambda$. Write $\omega_{i} \in \Lambda^{+}$for the fundamental coweight of $G$ corresponding to the representation $\bigwedge^{i} V^{\alpha}$ of $\mathrm{SO}_{2 n+1}, i=1, \ldots, n$. Let $\operatorname{Loc}: \operatorname{Rep}(\check{Q}) \rightarrow \operatorname{Sph}\left(\mathrm{Gr}_{Q}\right)^{\natural}$ denote the Satake equivalence, normalized to send an irreducible representation of $\check{Q}$ with highest weight $\mu$ to $\mathcal{A}_{Q, \mu}$.

Proposition 15. - Let $\lambda \in \Lambda^{+}$and $\theta$ be the image of $\lambda$ in $\Lambda_{G, P}$. Then $F^{\theta}\left(\mathcal{A}_{\lambda}\right) \widetilde{\longrightarrow} \operatorname{Loc}\left(V_{\theta}^{\lambda}\right)$ canonically. In particular, $F^{\theta}\left(\mathcal{A}_{\omega_{i}}\right) \stackrel{\rightrightarrows}{\rightarrow} \mathcal{A}_{Q, \omega_{i}}$ for $\left\langle\theta, \check{\omega}_{n}\right\rangle=i$.

4 SÉRIE - TOME $39-2006-\mathrm{N}^{\circ} 3$ 
Proof. - We could similarly define the functor $F^{\theta}: \operatorname{Sph}\left(\mathrm{Gr}_{G}\right) \rightarrow \operatorname{Sph}\left(\mathrm{Gr}_{Q}^{\theta}\right)$. Write $\mathcal{A}_{\lambda, \text { old }}$ for the corresponding object of $\operatorname{Sph}\left(\mathrm{Gr}_{G}\right)$. We claim that $F^{\theta}\left(\mathcal{A}_{\lambda}\right) \widetilde{\longrightarrow} F^{\theta}\left(\mathcal{A}_{\lambda, \text { old }}\right)$ canonically for our particular $\theta$.

Indeed, $S_{P}^{\theta} \cap \overline{\mathrm{Gr}}_{G}^{\lambda} \hookrightarrow \overline{\mathrm{Gr}}_{G}^{\lambda}$ is an open immersion, and the gerbe $\widetilde{S}_{P}^{\theta} \rightarrow S_{P}^{\theta}$ is trivial. So, the $*$-restriction of $\mathcal{A}_{\lambda}$ under $S_{P}^{\theta} \cap \overline{\mathrm{Gr}}_{G}^{\lambda} \rightarrow \widetilde{\mathrm{Gr}}_{G}$ is the Goresky-MacPherson extension from $S_{P}^{\theta} \cap \mathrm{Gr}_{G}^{\lambda}$. The assertion follows now from [5, Proposition 4.3.3 and Theorem 4.3.4].

PROPOSITION 16. -

(i) If $1 \leqslant i \leqslant n$ then $\mathcal{A}_{\omega_{i}}$ appears in $\mathcal{A}_{\alpha}^{\otimes i}$.

(ii) For $\lambda, \mu \in \Lambda$ the multiplicity of $\mathcal{A}_{\lambda+\mu}$ in $\mathcal{A}_{\lambda} \otimes \mathcal{A}_{\mu}$ is one.

Proof. - (i) Let $\theta \in \Lambda_{G, P}$ be given by $\left\langle\theta, \check{\omega}_{n}\right\rangle=i$. By Proposition 14, $F\left(\mathcal{A}_{\alpha}^{\otimes i}\right) \widetilde{\rightrightarrows}$ $\left(\mathcal{A}_{Q, \alpha} \oplus \mathcal{A}_{Q,-\alpha}\right)^{\otimes i}$. So, $F^{\theta}\left(\mathcal{A}_{\alpha}^{\otimes i}\right) \stackrel{\sim}{\rightarrow} \mathcal{A}_{Q, \alpha}^{\otimes i}$. Applying an appropriate symmetrization functor (either invariants or anti-invariants), one gets a direct summand $\mathcal{V} \subset \mathcal{A}_{\alpha}^{\otimes i}$ such that $F^{\theta}(\mathcal{V}) \widetilde{\rightarrow} \mathcal{A}_{Q, \omega_{i}}$.

If $\mathcal{A}_{\lambda}$ appears in $\mathcal{V}$ then $F^{\theta}\left(\mathcal{A}_{\lambda}\right) \subset F^{\theta}(\mathcal{V})$, because $F^{\theta}$ is exact. Besides, $\lambda \leqslant i \alpha$ in the sense that $\operatorname{Gr}_{G}^{\lambda} \subset \overline{\operatorname{Gr}}_{G}^{i \alpha}$, so $\left\langle\lambda, \check{\omega}_{n}\right\rangle \leqslant i$. If $\left\langle\lambda, \check{\omega}_{n}\right\rangle<i$ then $F^{\theta}\left(\mathcal{A}_{\lambda}\right)=0$ by Remark 9 . If $\left\langle\lambda, \check{\omega}_{n}\right\rangle=i$ then, by Corollary $1, \mathcal{A}_{Q, \lambda}$ appears in $F^{\theta}(\mathcal{V}) \widetilde{\neg} \mathcal{A}_{Q, \omega_{i}}$, so $\lambda=\omega_{i}$. The assertion follows.

(ii) Consider the convolution map $m: \overline{\mathrm{Gr}}_{G}^{\lambda} \tilde{\times} \overline{\mathrm{Gr}}_{G}^{\mu} \rightarrow \overline{\mathrm{Gr}}_{G}^{\lambda+\mu}$ as in Section 8.2. Its restriction to the open subscheme $\operatorname{Gr}_{G}^{\lambda} \tilde{\times} \operatorname{Gr}_{G}^{\mu} \rightarrow \operatorname{Gr}_{G}^{\lambda+\mu}$ is an isomorphism, as follows from [20, Lemma 4.3 and formula 3.6]. We are done.

Proof of Proposition 14(ii). - Call an object $K \in \operatorname{Sph}\left(\widetilde{\mathrm{Gr}}_{G}\right)$ even (respectively, odd) if $F^{\theta}(K)=0$ unless $b(\theta)=0$ (respectively, $b(\theta)=1$ ). Proposition 11 combined with Proposition 16 shows that $\mathcal{A}_{\alpha}$ is a tensor generator of $\operatorname{Sph}\left(\widetilde{\mathrm{Gr}}_{G}\right)$. Since $\mathcal{A}_{\alpha}$ is odd, we get a $\mathbb{Z} / 2 \mathbb{Z}$-grading on $\operatorname{Sph}\left(\widetilde{\mathrm{Gr}}_{G}\right)$ compatible with the tensor structure. Moreover, $F^{\prime}$ is compatible with the gradings. The uniqueness of the $\mathbb{Z} / 2 \mathbb{Z}$-grading is clear, because $\mathcal{A}_{\alpha}$ is irreducible.

DEFinition 6. - Let $\operatorname{Sph}\left(\widetilde{\mathrm{Gr}}_{G, x}\right)^{b}$ be the category of even objects in $\operatorname{Sph}\left(\widetilde{\mathrm{Gr}}_{G, x}\right) \otimes \operatorname{Vect}^{\varepsilon}$.

By Proposition 14, we get a tensor functor $F^{\prime}: \operatorname{Sph}\left(\widetilde{\operatorname{Gr}}_{G, x}\right)^{b} \rightarrow \operatorname{Sph}^{\prime}\left(\operatorname{Gr}_{Q, x}\right)$. Denote by $F^{\natural}$ the composition

$$
\operatorname{Sph}\left(\widetilde{\mathrm{Gr}}_{G}\right)^{b} \stackrel{F^{\prime}}{\longrightarrow} \operatorname{Sph}^{\prime}\left(\mathrm{Gr}_{Q}\right) \stackrel{\rightarrow}{\rightarrow} \operatorname{Sph}\left(\mathrm{Gr}_{Q}\right)^{\natural} .
$$

Let $\tilde{h}: \operatorname{Sph}\left(\widetilde{\operatorname{Gr}}_{G}\right)^{b} \rightarrow$ Vect denote the tensor functor $\tilde{h}=h \circ F^{\natural}$.

COROLlaRY 2. - There is an affine group scheme $\check{G}$ over $\overline{\mathbb{Q}}_{\ell}$ such that $\operatorname{Sph}\left(\widetilde{\mathrm{Gr}}_{G}\right)^{b}$ and the category $\operatorname{Rep}(\breve{G})$ of $\overline{\mathbb{Q}}_{\ell}$-representations of $\breve{G}$ are canonically equivalent as tensor categories.

Proof. - By Corollary 1, for each nonzero $\lambda \in \Lambda^{+}$the rank of $\tilde{h}\left(\mathcal{A}_{\lambda}\right)$ is at least 2. By [10, Proposition 1.20], $\operatorname{Sph}\left(\widetilde{\mathrm{Gr}}_{G}\right)^{b}$ is a rigid Abelian tensor category (cf. Definition 1.7, loc.cit) and $\tilde{h}: \operatorname{Sph}\left(\widetilde{\mathrm{Gr}}_{G}\right)^{b} \rightarrow$ Vect is a fibre functor. Our assertion follows now from (Theorem 2.11, loc.cit.).

Write $W^{\lambda}$ for the representation of $\check{G}$ corresponding to $\mathcal{A}_{\lambda}, \lambda \in \Lambda^{+}$. The functor $F^{\natural}: \operatorname{Sph}\left(\widetilde{\mathrm{Gr}}_{G}\right)^{b} \rightarrow \operatorname{Sph}\left(\mathrm{Gr}_{Q}\right)^{\natural}$ yields a morphism $\check{Q} \rightarrow \check{G}$. By Proposition $13, W^{\alpha}=U^{\alpha} \oplus U^{\alpha *}$, where $U^{\alpha}$ is the irreducible representation of $\check{Q}$ of highest weight $\alpha$. Since $W^{\alpha}$ is a faithful representation of $\check{Q}$, it follows that $\check{Q} \rightarrow \check{G}$ is an injection.

Since $W^{\alpha}$ is a tensor generator of $\operatorname{Sph}\left(\widetilde{G r}_{G}\right)^{b}, \check{G}$ is of finite type. We also get that $\check{G} \subset$ $\operatorname{SL}\left(W^{\alpha}\right)$. Indeed, the only object of rank one in $\operatorname{Sph}\left(\widetilde{G r}_{G}\right)^{b}$ is $\mathcal{A}_{0}$, so $\breve{G}$ acts trivially on $\operatorname{det} W^{\alpha}$.

Let $\mathcal{S} \in \operatorname{Rep}(\check{G})$ be such that the strictly full subcategory of $\operatorname{Rep}(\check{G})$, whose objects are isomorphic to subobjects of $\bigoplus_{i=1}^{m} \mathcal{S}$, is stable under the tensor structure. Then $\check{Q}$ acts trivially on 
$F^{\natural}(\mathcal{S})$, because $\check{Q}$ is connected. If $\check{Q}$ acts trivially on some $F^{\natural}\left(\mathcal{A}_{\lambda}\right)$ then $\lambda=0$ by Proposition 15 . So, $\mathcal{S}$ is a multiple of $\mathcal{A}_{0}$. By [10, 2.22], this implies that $\breve{G}$ is connected. Now by (loc.cit., 2.23), $\check{G}$ is reductive.

The above $\mathbb{Z} / 2 \mathbb{Z}$-grading on $\operatorname{Sph}\left(\widetilde{\mathrm{Gr}}_{G}\right)^{b}$ gives rise to a group homomorphism $\mu_{2} \rightarrow \check{G}$.

LEMMA 13. - For $i=1, \ldots, n$ the multiplicity of $W^{\omega_{i}}$ in $\bigwedge^{i} W^{\alpha}$ is one. If $W^{\lambda}$ appears in $\bigwedge^{i} W^{\alpha}$ and $\lambda \neq \omega_{i}$ then $\left\langle\lambda, \check{\omega}_{n}\right\rangle<i$.

Proof. - Let $\theta \in \Lambda_{G, P}$ be given by $\left\langle\theta, \check{\omega}_{n}\right\rangle=i$. The direct summand of $\bigwedge^{i} W^{\alpha}=$ $\bigwedge^{i}\left(U^{\alpha} \oplus U^{\alpha *}\right)$, on which $Z(\check{Q})$ acts by $\theta$ is $\bigwedge^{i} U^{\alpha}$. It follows that $F^{\theta}\left(\bigwedge^{i} \mathcal{A}_{\alpha}\right)=\mathcal{A}_{Q, \omega_{i}}$, where we denoted by $\bigwedge^{i} \mathcal{A}_{\alpha}$ the object of $\operatorname{Sph}\left(\widetilde{\mathrm{Gr}}_{G}\right)^{b}$ corresponding to $\bigwedge^{i} W^{\alpha}$.

If $W^{\lambda}$ appears in $\bigwedge^{i} W^{\alpha}$ then $F^{\theta}\left(\mathcal{A}_{\lambda}\right) \subset F^{\theta}\left(\bigwedge^{i} \mathcal{A}_{\alpha}\right)$, because $F^{\theta}$ is exact. Besides, $\lambda \leqslant i \alpha$ in the sense that $\operatorname{Gr}_{G}^{\lambda} \subset \overline{\mathrm{Gr}}_{G}^{i \alpha}$, so $\left\langle\lambda, \check{\omega}_{n}\right\rangle \leqslant i$. If $\left\langle\lambda, \check{\omega}_{n}\right\rangle<i$ then $F^{\theta}\left(\mathcal{A}_{\lambda}\right)=0$ by Remark 9 . If $\left\langle\lambda, \check{\omega}_{n}\right\rangle=i$ then, by Corollary $1, \mathcal{A}_{Q, \lambda}$ appears in $F^{\theta}\left(\bigwedge^{i} \mathcal{A}_{\alpha}\right)=\mathcal{A}_{Q, \omega_{i}}$, so $\lambda=\omega_{i}$. The assertion follows.

Proof of Theorem 3. - Step 1. Let us show that $\mathcal{A}_{\alpha} * \mathcal{A}_{\alpha} \widetilde{\rightarrow} \mathcal{A}_{2 \alpha} \oplus \mathcal{A}_{\omega_{2}} \oplus \mathcal{A}_{0}$ for $n \geqslant 2$ and $\mathcal{A}_{\alpha} * \mathcal{A}_{\alpha} \widetilde{\rightarrow} \mathcal{A}_{2 \alpha} \oplus \mathcal{A}_{0}$ for $n=1$. Indeed, by Proposition 16, $\mathcal{A}_{2 \alpha} \oplus \mathcal{A}_{\omega_{2}}$ appears in $\mathcal{A}_{\alpha} * \mathcal{A}_{\alpha}$. Let $\theta \in \Lambda_{G, P}$ be given by $\left\langle\theta, \check{\omega}_{n}\right\rangle=2$. By Proposition $15, F^{\theta}\left(\mathcal{A}_{2 \alpha}\right) \stackrel{\mathcal{\omega}_{2}}{\rightarrow} \mathcal{A}_{Q, 2 \alpha}$ and $F^{\theta}\left(\mathcal{A}_{\omega_{2}}\right) \stackrel{\sim}{\rightarrow} \mathcal{A}_{Q, \omega_{2}}$. We have

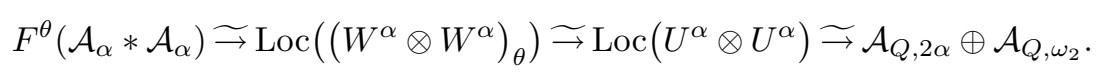

So, $\mathcal{A}_{\alpha} * \mathcal{A}_{\alpha} \widetilde{\rightarrow} \mathcal{A}_{2 \alpha} \oplus \mathcal{A}_{\omega_{2}} \oplus K$ for some $K \in \operatorname{Sph}\left(\widetilde{\mathrm{Gr}}_{G}\right)$ such that $F^{\theta^{\prime}}(K)=0$ unless $\left\langle\theta^{\prime}, \check{\omega}_{n}\right\rangle<2$. Since $\mathcal{A}_{\alpha}$ is odd, $\mathcal{A}_{\alpha} * \mathcal{A}_{\alpha}$ is even, so $K$ is multiple of $\mathcal{A}_{0}$. The desired assertion follows now from $\operatorname{Hom}\left(\mathcal{A}_{0}, \mathcal{A}_{\alpha} * \mathcal{A}_{\alpha}\right) \stackrel{\longrightarrow}{\rightarrow} \operatorname{Hom}\left(\mathcal{A}_{\alpha}, \mathcal{A}_{\alpha}\right) \widetilde{\hookrightarrow} \overline{\mathbb{Q}}_{\ell}$.

Step 2. Let us show that $\mathcal{A}_{0}$ appears in $\bigwedge^{2} \mathcal{A}_{\alpha}$. Assume the contrary, that is, $\mathcal{A}_{0}$ appears in $\operatorname{Sym}^{2} \mathcal{A}_{\alpha}$. Then $n \geqslant 2$ and $\check{G} \subset \mathrm{SO}\left(W^{\alpha}\right)$ for the symmetric form $\operatorname{Sym}^{2} W^{\alpha} \rightarrow$ $U^{\alpha} \otimes U^{\alpha *} \rightarrow \overline{\mathbb{Q}}_{\ell}$.

Let $\check{U}$ (respectively, $\check{U}^{-}$) denote the unipotent radical of the Siegel parabolic $\check{P} \subset \mathrm{S} \mathbb{O}\left(W^{\alpha}\right)$ (respectively, $\check{P}^{-} \subset \mathrm{SO}\left(W^{\alpha}\right)$ ) preserving the isotropic subspace $U^{\alpha} \subset W^{\alpha}$ (respectively, $\left.U^{\alpha *} \subset W^{\alpha}\right)$. The Lie algebra Lie $\check{G}$ is a $\check{Q}$-subrepresentation of

$$
\mathfrak{s o}\left(W^{\alpha}\right)=\mathfrak{g l}\left(U^{\alpha}\right) \oplus \operatorname{Lie}(\check{U}) \oplus \operatorname{Lie}\left(\check{U}^{-}\right) .
$$

Since Lie $\check{U}$ and Lie $\check{U}^{-}$are irreducible $\check{Q}$-modules, $\check{G}$ coincides with one of the groups $\check{Q}, \check{P}, \check{P}^{-}, \mathrm{S} \mathbb{O}\left(W^{\alpha}\right)$. Since $\check{G}$ is reductive, it is either $\check{Q}$ or $\mathrm{SO}\left(W^{\alpha}\right)$. Since $W^{\alpha}$ is not irreducible as a representation of $\check{Q}, \check{G} \neq \check{Q}$, hence $\breve{G}=\mathrm{S} \mathbb{O}\left(W^{\alpha}\right)$.

Now Lemma 13 shows that $\bigwedge^{n} W^{\alpha} \widetilde{\hookrightarrow} W^{\omega_{n}} \oplus W^{\lambda}$ for some $\lambda \in \Lambda^{+}$with $\left\langle\lambda, \check{\omega}_{n}\right\rangle<n$. Let $\widetilde{U}$ denote the kernel of the contraction map $\bigwedge^{n-1} U^{\alpha} \otimes U^{\alpha *} \rightarrow \bigwedge^{n-2} U^{\alpha}$, this is an irreducible $\check{Q}$-module. By the representation theory for $\mathrm{SO}_{2 n}$, we have

- $\widetilde{U} \subset W^{\lambda} \subset \Lambda^{n}\left(U^{\alpha} \oplus U^{\alpha *}\right)$ as $\check{Q}$-modules;

- if a weight $\theta$ of $Z(\check{Q})$ appears in $W^{\lambda}$ then $\left\langle\theta, \check{\omega}_{n}\right\rangle \leqslant n-2$;

- for $\left\langle\theta, \check{\omega}_{n}\right\rangle=n-2$ the direct summand of $W^{\lambda}$ on which $Z(\check{Q})$ acts by $\theta$ is $\widetilde{U}$.

Let $\theta$ be the image of $\lambda$ in $\Lambda_{G, P}$, we get $F^{\theta}\left(\mathcal{A}_{\lambda}\right) \widetilde{\rightrightarrows} \widetilde{U}$. By Corollary $1, \mathcal{A}_{Q, \lambda} \widetilde{\rightrightarrows} \widetilde{U}$. However, the highest weight of $\widetilde{U}$ does not lie in $\Lambda_{+}$. This contradiction yields our statement.

Step 3. We know already that $\breve{G} \subset \operatorname{Sp}\left(W^{\alpha}\right)$ for the form $\bigwedge^{2} W^{\alpha} \rightarrow U^{\alpha} \otimes U^{\alpha *} \rightarrow \overline{\mathbb{Q}}_{\ell}$. Let $\check{P} \subset \mathbb{S p}\left(W^{\alpha}\right)$ (respectively, $\check{P}^{-} \subset \mathbb{S p}\left(W^{\alpha}\right)$ ) denote the Siegel parabolic preserving the Lagrangian subspace $U^{\alpha} \subset W^{\alpha}$ (respectively, $U^{\alpha *} \subset W^{\alpha}$ ). As in step 2, one shows that $\check{G}$

$4^{e}$ SÉRIE - TOME $39-2006-\mathrm{N}^{\circ} 3$ 
coincides with one of the groups $\check{Q}, \check{P}, \check{P}^{-}, \operatorname{Sp}\left(W^{\alpha}\right)$. Since $\check{G}$ is reductive, it is either $\check{Q}$ or $\operatorname{Sp}\left(W^{\alpha}\right)$. The $\check{Q}$-representation $W^{\alpha}$ is not irreducible, so $\check{G}=\operatorname{Sp}\left(W^{\alpha}\right)$.

\section{Hecke operators}

9.1. According to A.3, inside of $\mathrm{D}\left(\widetilde{\mathrm{Bun}}_{G}\right)$ we have the full triangulated subcategories $\mathrm{D}_{ \pm}\left(\widetilde{\operatorname{Bun}_{G}}\right)$. Let us define for each $K \in \operatorname{Sph}\left(\widetilde{\mathrm{Gr}}_{G}\right)$ a Hecke operator $\mathrm{H}(K, \cdot): \mathrm{D}(\widetilde{\mathrm{Bun}} G) \rightarrow$ $\mathrm{D}\left(X \times \widetilde{\operatorname{Bun}}_{G}\right)$ sending $\mathrm{D}_{ \pm}\left(\widetilde{\operatorname{Bun}_{G}}\right)$ to $\mathrm{D}_{ \pm}\left(X \times \widetilde{\operatorname{Bun}_{G}}\right)$.

Denote by $\mathcal{H}_{G}$ the Hecke stack classifying $\left(\mathcal{F}_{G}, \mathcal{F}_{G}^{\prime}, x \in X, \beta\right)$, where $\mathcal{F}_{G}, \mathcal{F}_{G}^{\prime}$ are $G$-torsors on $X$, and $\beta:\left.\mathcal{F}_{G} \underset{\rightarrow}{\longrightarrow} \mathcal{F}_{G}^{\prime}\right|_{X-x}$ is an isomorphism. We have the diagram

$$
\operatorname{Bun}_{G} \stackrel{p}{\stackrel{H}{H}} \mathcal{H}_{G} \stackrel{p^{\prime}}{\longrightarrow} \operatorname{Bun}_{G},
$$

where $p$ (respectively, $p^{\prime}$ ) sends the above point to $\mathcal{F}_{G}$ (respectively, to $\mathcal{F}_{G}^{\prime}$ ). Let $\widetilde{\mathcal{H}}_{G}$ be the stack obtained from $\widetilde{\operatorname{Bun}}_{G} \times \widetilde{\operatorname{Bun}}_{G}$ by the base change $\mathcal{H}_{G} \stackrel{p, p^{\prime}}{\longrightarrow} \operatorname{Bun}_{G} \times \operatorname{Bun}_{G}$. Denote by $\tilde{p}, \tilde{p}^{\prime}$ the projections that fit into the diagram

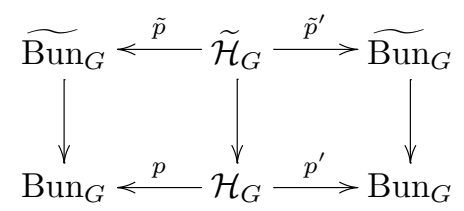

Recall that the 'trivial' $G$-torsor $\mathcal{F}_{G}^{0}$ on $X$ is given by $M_{0}=\mathcal{O}_{X}^{n} \oplus \Omega^{n}$. Write $\operatorname{Bun}_{G, X}$ for the stack classifying triples $\left(\mathcal{F}_{G}, x \in X, \nu\right)$, where $\mathcal{F}_{G} \in \operatorname{Bun}_{G}$ and $\nu:\left.\mathcal{F}_{G} \widetilde{\rightarrow} \mathcal{F}_{G}^{0}\right|_{D_{x}}$ is a trivialization over the formal disk $D_{x}$ at $x \in X$. Then $\operatorname{Bun}_{G, X}$ is a $G_{X}$-torsor over $X \times \operatorname{Bun}_{G}$. Set $\widetilde{\operatorname{Bun}}_{G, X}=\widetilde{\operatorname{Bun}}_{G} \times_{\mathrm{Bun}_{G}} \operatorname{Bun}_{G, X}$.

Denote by $\gamma$ (respectively, $\gamma^{\prime}$ ) the isomorphism $\operatorname{Bun}_{G, X} \times_{G_{X}} \operatorname{Gr}_{G, X} \widetilde{\rightarrow} \mathcal{H}_{G}$ such that the projection to the first term corresponds to $p$ (respectively, to $p^{\prime}$ ). Recall the line bundle $\mathcal{A}$ on $\operatorname{Bun}_{G}$ (cf. 3.2). We have canonically

$$
\gamma^{\prime *} p^{*} \mathcal{A} \underset{\rightarrow}{\mathcal{A} \widetilde{\otimes} \mathcal{L}^{-1}}
$$

This yields a $G_{X}$-torsor $\widetilde{\operatorname{Bun}}_{G, X} \times \widetilde{\mathrm{Gr}}_{G, X} \rightarrow \widetilde{\mathcal{H}}_{G}$ extending the $G_{X}$-torsor

$$
\operatorname{Bun}_{G, X} \times \operatorname{Gr}_{G, X} \rightarrow \operatorname{Bun}_{G, X} \times_{G_{X}} \operatorname{Gr}_{G, X} \stackrel{\gamma^{\prime}}{\longrightarrow} \mathcal{H}_{G} .
$$

So, for $\mathcal{S} \in \operatorname{Sph}\left(\widetilde{\operatorname{Gr}}_{G, X}\right)$ and $\mathcal{T} \in \mathrm{D}\left(\widetilde{\operatorname{Bun}}_{G}\right)$ we can form their twisted tensor product $\mathcal{T} \widetilde{\otimes} \mathcal{S} \in \mathrm{D}\left(\widetilde{\mathcal{H}}_{G}\right)$. Set

$$
\mathrm{H}(\mathcal{S}, \mathcal{T})=(\operatorname{supp} \times \tilde{p}) !(\mathcal{T} \widetilde{\otimes} \mathcal{S})
$$

where supp : $\widetilde{\mathcal{H}}_{G} \rightarrow X$ is the projection. In a similar way, for any $\mathcal{S} \in \operatorname{Sph}\left(\widetilde{\operatorname{Gr}}_{G, X^{d}}\right)$ one defines the functor $\mathrm{H}(\mathcal{S}, \cdot): \mathrm{D}\left(\widetilde{\operatorname{Bun}}_{G}\right) \rightarrow \mathrm{D}\left(X^{d} \times \widetilde{\operatorname{Bun}}_{G}\right)$.

Recall the functor glob: $\operatorname{Sph}\left(\widetilde{\mathrm{Gr}}_{G}\right) \rightarrow \operatorname{Sph}\left(\widetilde{\mathrm{Gr}}_{G, X}\right)$ (cf. 8.3.1). For $K \in \operatorname{Sph}\left(\widetilde{\mathrm{Gr}}_{G}\right)$ set $\mathrm{H}(K, \mathcal{T})=\mathrm{H}(\operatorname{glob}(K), \mathcal{T})$. 
The Hecke functors commute with Verdier duality $\mathbb{D H}(K, \mathcal{T}) \stackrel{\rightarrow}{\rightarrow}(\mathbb{D} K, \mathbb{D} \mathcal{T})$, because $\operatorname{Gr}_{G}$ is ind-proper. Besides, they are compatible with the convolution product on $\operatorname{Sph}\left(\widetilde{\mathrm{Gr}}_{G}\right)$, namely, for $\mathcal{S}_{1}, \mathcal{S}_{2} \in \operatorname{Sph}\left(\widetilde{\operatorname{Gr}}_{G, X}\right)$ we have canonically $\mathrm{H}\left(\mathcal{S}_{2}, \mathrm{H}\left(\mathcal{S}_{1}, \mathcal{T}\right)\right) \underset{\rightarrow}{\longrightarrow}\left(\mathcal{S}_{1} *_{X} \mathcal{S}_{2}, \mathcal{T}\right)$.

The geometric Langlands program for the metaplectic group would be a trial to understand the action of $\operatorname{Sph}\left(\widetilde{\mathrm{Gr}}_{G}\right)^{b}$ on $\mathrm{D}_{-}\left(\widetilde{\mathrm{Bun}}_{G}\right)$, that is, to look for automorphic sheaves or, more generally, for a 'spectral decomposition' of $\mathrm{D}_{-}\left(\widetilde{\mathrm{Bun}}_{G}\right)$ under this action.

Recall that the metaplectic representation is automorphic. In the geometric setting this is reflected in the following Hecke property of Aut. Set

$$
\mathrm{St}=\overline{\mathbb{Q}}_{\ell}[2 n-1]\left(\frac{2 n-1}{2}\right) \oplus \overline{\mathbb{Q}}_{\ell}[2 n-3]\left(\frac{2 n-3}{3}\right) \oplus \cdots \oplus \overline{\mathbb{Q}}_{\ell}[1-2 n]\left(\frac{1-2 n}{2}\right),
$$

so St has cohomologies in odd degrees only and $\mathbb{D}(\mathrm{St}) \stackrel{\rightarrow}{\rightarrow} \mathrm{St}$ as a complex over Spec $k$.

THEOREM 4. - Over $X \times \widetilde{\operatorname{Bun}}_{G}$ we have

$$
\begin{aligned}
& \mathrm{H}\left(\mathcal{A}_{\alpha}, \mathrm{Aut}_{g}\right) \stackrel{\sim}{\rightarrow} \mathrm{St}[1]\left(\frac{1}{2}\right) \otimes \mathrm{Aut}_{s}, \\
& \mathrm{H}\left(\mathcal{A}_{\alpha}, \mathrm{Aut}_{s}\right) \stackrel{\sim}{\rightarrow} \mathrm{St}[1]\left(\frac{1}{2}\right) \otimes \mathrm{Aut}_{g} .
\end{aligned}
$$

\subsection{Proof of Theorem 4}

Let $\mathcal{H}_{G}^{\alpha} \subset \mathcal{H}_{G}$ be the locally closed substack given by the condition that $\mathcal{F}_{G}$ is in the position $\alpha$ with respect to $\mathcal{F}_{G}^{\prime}$ (or, equivalently, $\mathcal{F}_{G}^{\prime}$ is in the position $\alpha$ with respect to $\mathcal{F}_{G}$ ). Set $\widetilde{\mathcal{H}}_{G}^{\alpha}=\mathcal{H}_{G}^{\alpha} \times \mathcal{H}_{G} \widetilde{\mathcal{H}}_{G}$.

LEMMA 14. - There exist isomorphisms

$$
\kappa, \kappa^{\prime}: \widetilde{\mathcal{H}}_{G}^{\alpha} \widetilde{\longrightarrow}\left(\widetilde{\operatorname{Bun}}_{G} \times{\mathrm{Bun}_{G}}_{G}^{\alpha}\right) \times B\left(\mu_{2}\right),
$$

where we used $p: \mathcal{H}_{G}^{\alpha} \rightarrow \operatorname{Bun}_{G}$ (respectively, $p^{\prime}: \mathcal{H}_{G}^{\alpha} \rightarrow \operatorname{Bun}_{G}$ ) in the fibred product, and the projection to the first term corresponds to $\tilde{p}: \widetilde{\mathcal{H}}_{G}^{\alpha} \rightarrow \widetilde{\operatorname{Bun}_{G}}$ (respectively, to $\tilde{p}^{\prime}: \widetilde{\mathcal{H}}_{G}^{\alpha} \rightarrow \widetilde{\operatorname{Bun}}_{G}$ ).

Proof. - A point of $\widetilde{\mathcal{H}}_{G}^{\alpha}$ is given by $\left(\mathcal{F}_{G}, \mathcal{F}_{G}^{\prime}, x \in X, \beta\right) \in \mathcal{H}_{G}^{\alpha}$, two 1-dimensional vector

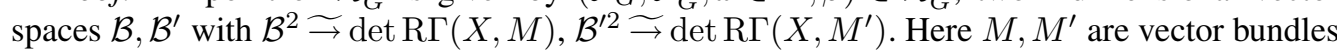
on $X$ obtained from $\mathcal{F}_{G}, \mathcal{F}_{G}^{\prime}$ via the standard representation of $G$.

The symplectic form on $M$ induces a perfect pairing $\left(M+M^{\prime}\right) / M \otimes\left(M+M^{\prime}\right) / M^{\prime} \rightarrow$ $\Omega(x) / \Omega \widetilde{\rightrightarrows} k$ between these 1 -dimensional spaces. Further,

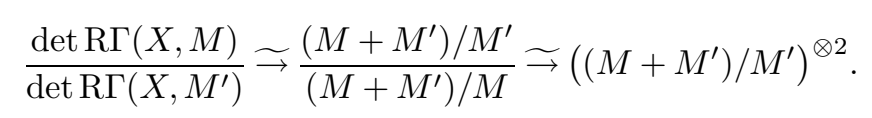

Instead of providing $\mathcal{B}, \mathcal{B}^{\prime}$ we may provide $\mathcal{B}, \mathcal{B}_{0}$, where $\operatorname{dim} \mathcal{B}_{0}=1$, with an isomorphism $\mathcal{B}_{0}^{2} \widetilde{\rightrightarrows} k$, letting $\mathcal{B}^{\prime}=\mathcal{B} \otimes\left(\left(M+M^{\prime}\right) / M^{\prime}\right)^{*} \otimes \mathcal{B}_{0}$. This defines $\kappa$. The datum of $\mathcal{B}^{\prime}, \mathcal{B}_{0}$ defines $\kappa^{\prime}$.

As above, let $W$ denote the nontrivial local system of rank one on $B\left(\mu_{2}\right)$ corresponding to the covering Spec $k \rightarrow B\left(\mu_{2}\right)$. For the diagram

$$
X \times \widetilde{\operatorname{Bun}}_{G} \stackrel{\operatorname{supp} \times \tilde{p}}{\longleftarrow} \widetilde{\mathcal{H}}_{G}^{\alpha} \stackrel{\tilde{p}^{\prime}}{\longrightarrow} \widetilde{\operatorname{Bun}}_{G}
$$

the Hecke operator writes $\mathrm{H}\left(\mathcal{A}_{\alpha}, K\right) \stackrel{\Im}{\rightarrow}(\operatorname{supp} \times \tilde{p}) !\left(\tilde{p}^{*} K \otimes \kappa^{*} W\right)[2 n+1]\left(\frac{2 n+1}{2}\right)$.

$4^{\text {e }}$ SÉRIE - TOME $39-2006-\mathrm{N}^{\circ} 3$ 


\subsubsection{Stratifications}

Let $(x, M)$ be a $k$-point of $X \times{ }_{i} \operatorname{Bun}_{G}$. Denote by $Y$ the fibre of supp $\times p: \mathcal{H}_{G}^{\alpha} \rightarrow X \times \operatorname{Bun}_{G}$ over $(x, M)$. So, $Y$ can be identified with the variety $\bar{Z}-A$ of Section 8.5 . Let $Y_{k}$ denote the preimage of ${ }_{k} \operatorname{Bun}_{G}$ under $Y \hookrightarrow \mathcal{H}_{G}^{\alpha} \stackrel{p^{\prime}}{\longrightarrow} \operatorname{Bun}_{G}$. We are going to describe the stratification of $Y$ by the subschemes $Y_{k}$.

Recall that $M \in \operatorname{Bun}_{2 n}$ with symplectic form $\bigwedge^{2} M \rightarrow \Omega$ and $\operatorname{dim} \mathrm{H}^{0}(M)=i$ (for brevity, in this subsection we omit the argument $X$ in the cohomology groups). For a $k$-point $M^{\prime}$ of $Y$ we get

$$
\begin{array}{cccccc} 
& M & \subset & M+M^{\prime} & \subset & M(x) \\
& \cup & & \cup & & \\
M(-x) & \subset & M \cap M^{\prime} & \subset & M^{\prime} &
\end{array}
$$

and $\left.\operatorname{dim}\left(M+M^{\prime}\right) / M=1, \operatorname{dim}\left(M \cap M^{\prime}\right) / M(-x)\right)=2 n-1$. Actually, $\left(M \cap M^{\prime}\right) / M(-x)$ is the orthogonal complement to $\left(M+M^{\prime}\right) / M$ for the perfect pairing

$$
M(x) / M \otimes M / M(-x) \rightarrow \Omega(x) / \Omega \widetilde{\rightarrow} k
$$

induced by the form on $M$. Let $\pi: Y \rightarrow V=\mathbb{P}(M(x) / M)$ be the map sending $M^{\prime}$ to the line $M+M^{\prime} / M$. Let $N$ be the image of $\mathrm{H}^{0}(M) \rightarrow M / M(-x)$. Set $j=\operatorname{dim} N$, so $\operatorname{dim} \mathrm{H}^{0}(M(-x))=i-j$. Since $M \widetilde{\longrightarrow} M^{*} \otimes \Omega$,

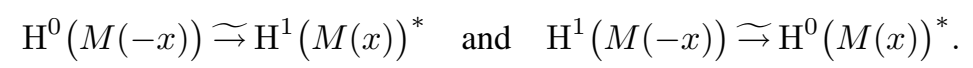

The long exact sequence

$$
0 \rightarrow \mathrm{H}^{0}(M) \rightarrow \mathrm{H}^{0}(M(x)) \rightarrow M(x) / M \rightarrow \mathrm{H}^{1}(M) \rightarrow \mathrm{H}^{1}(M(x)) \rightarrow 0
$$

shows that $\operatorname{dim} \mathrm{H}^{0}(M(x))=i+2 n-j$, because $\operatorname{dim} \mathrm{H}^{1}(M(x))=i-j$. We have

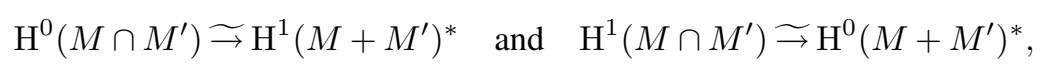

because $\left(M+M^{\prime}\right)^{*} \otimes \Omega \widetilde{\rightarrow} M \cap M^{\prime}$. Note that $\chi\left(M \cap M^{\prime}\right)=-1$ and $\chi\left(M+M^{\prime}\right)=1$.

We distinguish three cases

(0) $j=0$. So, $\mathrm{H}^{0}(M(-x))=\mathrm{H}^{0}(M)$ is $i$-dimensional and $\operatorname{dim} \mathrm{H}^{0}(M(x))=2 n$. Then $\mathrm{H}^{0}(M(-x)) \stackrel{\sim}{\rightarrow} \mathrm{H}^{0}\left(M \cap M^{\prime}\right)$ is of dimension $i$, and $\operatorname{dim} \mathrm{H}^{0}\left(M+M^{\prime}\right)=i+1$. Clearly, for $M+M^{\prime} \in \mathbb{P}(M(x) / M)$ fixed we get a 1-dimensional subspace in $\left(M+M^{\prime}\right) /\left(M \cap M^{\prime}\right)$ generated by $\mathrm{H}^{0}\left(M+M^{\prime}\right)$. So, for $M+M^{\prime} \in V$ fixed there is a unique $M^{\prime}$ with $\operatorname{dim} \mathrm{H}^{0}\left(M^{\prime}\right)=i+1$ and for the other $M^{\prime}$ we have $\operatorname{dim} \mathrm{H}^{0}\left(M^{\prime}\right)=i$.

Thus, $\pi: Y \rightarrow V$ has a section $V \rightarrow Y$, which is the closed stratum $Y_{i+1}$. Its complement is the open stratum $Y_{i}$.

(1) $0<j<2 n$. View $V$ as the space of hyperplanes in $M / M(-x)$. We get a nontrivial subspace $V^{\prime} \subset V$ of hyperplanes that contain $N$. Distinguish two cases:

Case (1a). $N \subset\left(M \cap M^{\prime}\right) / M(-x)$ then $\mathrm{H}^{0}\left(M \cap M^{\prime}\right)=\mathrm{H}^{0}(M)$ is of dimension $i$, so $\operatorname{dim} \mathrm{H}^{0}\left(M+M^{\prime}\right)=i+1$. In the fibre of $\pi: Y \rightarrow V$ over $M+M^{\prime} / M$ we get a distinguished point corresponding to the subspace of $\left(M+M^{\prime}\right) /\left(M \cap M^{\prime}\right)$ generated by $\mathrm{H}^{0}\left(M+M^{\prime}\right)$. This point lies in ${ }_{i+1} \mathrm{Bun}_{G}$, and the complement lies in ${ }_{i} \operatorname{Bun}_{G}$.

Case (1b). $N \nsubseteq\left(M \cap M^{\prime}\right) / M(-x)$. Then $N \cap\left(M \cap M^{\prime}\right)$ is of dimension $j-1$. So, $\operatorname{dim} \mathrm{H}^{0}\left(M \cap M^{\prime}\right)=i-1$ and $\operatorname{dim}^{0}\left(M+M^{\prime}\right)=i$. Since $M^{\prime} \neq M$, we get $M^{\prime} \in{ }_{i-1} \operatorname{Bun}_{G}$. 
So, $Y$ has three nonempty strata in case (1). The map $\pi: \pi^{-1}\left(V^{\prime}\right) \rightarrow V^{\prime}$ has a section, which is the closed stratum $Y_{i+1} \underset{\rightarrow}{\rightarrow} V^{\prime}$. The complement to this section is the middle stratum $Y_{i}=\pi^{-1}\left(V^{\prime}\right)-V^{\prime}$, and the open stratum is $Y_{i-1}=\pi^{-1}\left(V-V^{\prime}\right)$.

(2) $j=2 n$. Then $\mathrm{H}^{0}(M)=\mathrm{H}^{0}(M(x))$ is $i$-dimensional, so $\operatorname{dim} \mathrm{H}^{0}\left(M+M^{\prime}\right)=i$ and $\operatorname{dim} \mathrm{H}^{0}\left(M \cap M^{\prime}\right)=i-1$. The image of $\mathrm{H}^{0}(M) \rightarrow\left(M+M^{\prime}\right) /\left(M \cap M^{\prime}\right)$ is 1-dimensional and equals $M /\left(M \cap M^{\prime}\right)$. So, $\operatorname{dim} \mathrm{H}^{0}\left(M^{\prime}\right)=i-1$, because $M^{\prime} \neq M$.

In this case $Y=Y_{i-1}$.

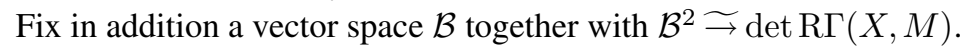

Proposition 17. - Let $K$ denote the fibre of $\mathrm{H}\left(\mathcal{A}_{\alpha}, \operatorname{Aut}_{g}\right)$ (respectively, of $\mathrm{H}\left(\mathcal{A}_{\alpha}, \mathrm{Aut}_{s}\right)$ ) at $(x, M, \mathcal{B}) \in X \times{ }_{i} \widetilde{\operatorname{Bun}}_{G}$. Then $K=0$ unless $i$ is odd (respectively, even). If $i$ is odd (respectively, even) then we have noncanonically $K \underset{\rightarrow}{\rightarrow} \mathrm{St}\left[1+d_{G}-i\right]$.

Proof. - (g) Consider the case where $K$ is the fibre of $\mathrm{H}\left(\mathcal{A}_{\alpha}\right.$, Aut $\left._{g}\right)$. Assume $i$ even, so only the stratum $Y_{i}$ of $Y$ contributes to $K$.

If $j=0$ then $Y_{i}$ is a $\mathbb{G}_{m}$-torsor over $V$, and the restriction of Aut $g$ to a fibre of $\pi: Y_{i} \rightarrow V$ is a nontrivial local system of order two, so $K=0$ in this case. If $j=2 n$ then $K=0$ because

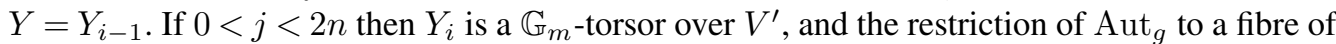
$\pi: Y_{i} \rightarrow V^{\prime}$ is a nontrivial local system of order two, so $K=0$.

Now let $i$ be odd, so only the strata $Y_{i-1}$ and $Y_{i+1}$ contribute to $K$.

If $j=0$ then the restriction of Aut $_{g}$ to $Y_{i+1}$ is isomorphic to $\overline{\mathbb{Q}}_{\ell}\left[d_{G}-i-1\right]$ by Theorem 1 , because $Y_{i+1} \underset{\rightarrow}{\longrightarrow} \mathbb{P}^{2 n-1}$ is simply-connected. Our assertion follows then from

$$
\mathrm{St} \underset{\rightarrow}{\sim} \Gamma\left(\mathbb{P}^{2 n-1}, \overline{\mathbb{Q}}_{\ell}\right)[2 n-1]\left(\frac{2 n-1}{2}\right) .
$$

If $j=2 n$ then the restriction of Aut to $_{i-1}$ is isomorphic to $\overline{\mathbb{Q}}_{\ell}\left[d_{G}-i+1\right]$, because $Y_{i-1}$ is simply-connected. So, $K \widetilde{\longrightarrow} \mathrm{St}\left[1+d_{G}-i\right]$. If $0<j<2 n$ then the restriction of $\operatorname{Aut}_{g}$ to $Y_{i+1}$ identifies with $\overline{\mathbb{Q}}_{\ell}\left[d_{G}-i-1\right]$, because $Y_{i+1} \underset{\rightarrow}{\rightarrow} V^{\prime}$ is simply-connected. The contribution of $Y_{i+1}$ to $K$ is

$$
\mathrm{R} \Gamma\left(V^{\prime}, \overline{\mathbb{Q}}_{\ell}\right)\left[d_{G}-i+2 n\right] .
$$

The restriction of $\mathrm{Aut}_{g}$ to $Y_{i-1}$ is $\overline{\mathbb{Q}}_{\ell}\left[d_{G}-i+1\right]$, because any rank one local system of order two on $\pi^{-1}\left(V-V^{\prime}\right)$ is trivial. So, the contribution of $Y_{i-1}$ to $K$ is $\mathrm{R}_{c}\left(V-V^{\prime}, \overline{\mathbb{Q}}_{\ell}\right)\left[d_{G}-i+2 n\right]$. The distinguished triangle

$$
\mathrm{R}_{c}\left(V-V^{\prime}, \overline{\mathbb{Q}}_{\ell}\right)\left[d_{G}-i+2 n\right] \rightarrow K \rightarrow \mathrm{R} \Gamma\left(V^{\prime}, \overline{\mathbb{Q}}_{\ell}\right)\left[d_{G}-i+2 n\right]
$$

yields the desired isomorphism.

(s) In the case where $K$ is the fibre of $\mathrm{H}\left(\mathcal{A}_{\alpha}, \mathrm{Aut}_{s}\right)$, the argument is similar.

9.2.2. For $k, r \geqslant 0$ denote by ${ }_{k, r} \mathcal{H}_{G}^{\alpha}$ the preimage of ${ }_{k} \operatorname{Bun}_{G} \times{ }_{r} \operatorname{Bun}_{G}$ under $p \times p^{\prime}: \mathcal{H}_{G}^{\alpha} \rightarrow$ $\operatorname{Bun}_{G} \times \operatorname{Bun}_{G}$. Similarly, define the stack $k, r \widetilde{\mathcal{H}}_{G}^{\alpha}$ by the Cartesian square

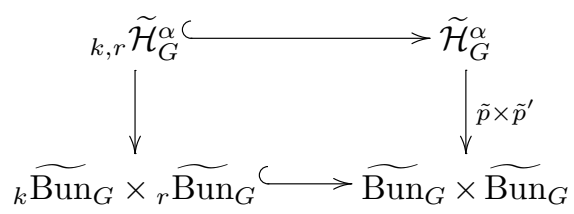

$4^{\text {e }}$ SÉRIE - TOME $39-2006-\mathrm{N}^{\circ} 3$ 
The two $S_{2}$-coverings over ${ }_{k, r} \widetilde{\mathcal{H}}_{G}^{\alpha}$ obtained from ${ }_{k} \rho: \operatorname{Cov}\left({ }_{k} \widetilde{\operatorname{Bun}}_{G}\right) \rightarrow{ }_{k} \widetilde{\operatorname{Bun}}_{G}$ and from ${ }_{r} \rho: \operatorname{Cov}\left({ }_{r} \widetilde{\operatorname{Bun}}_{G}\right) \rightarrow{ }_{r} \widetilde{\operatorname{Bun}}_{G}$ are canonically isomorphic, namely Lemma 14 implies the following.

LEMMA 15. - There is a canonical commutative diagram, where both squares are Cartesian

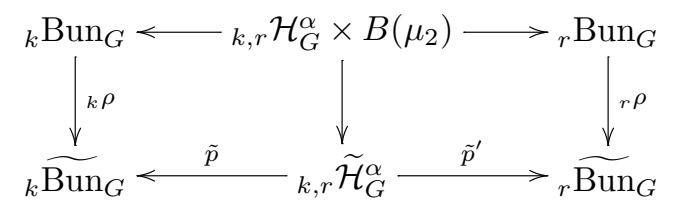

Let $\mathcal{U} \subset X \times{ }_{1} \operatorname{Bun}_{G}$ be the open substack given by $\mathrm{H}^{0}(X, M(-x))=0$. As in Lemma 1 , one shows that $\mathcal{U}$ is nonempty. In general, $\mathcal{U} \neq X \times{ }_{1} \operatorname{Bun}_{G}$. Let $\widetilde{\mathcal{U}}$ be the preimage of $\mathcal{U}$ in $X \times{ }_{1} \widetilde{\operatorname{Bun}}_{G}$.

PROPOSITION 18. - The first isomorphism of Theorem 4 holds over $\widetilde{\mathcal{U}}$, the second holds over $X \times{ }_{0} \widetilde{\operatorname{Bun}}_{G}$.

Proof. - $(\mathrm{g})$ Let $Y(\mathcal{U})$ be the preimage of $\mathcal{U}$ under supp $\times p: \mathcal{H}_{G}^{\alpha} \rightarrow X \times \operatorname{Bun}_{G}$. Write $Y_{k}(\mathcal{U})$ for the preimage of ${ }_{k} \operatorname{Bun}_{G}$ under $Y(\mathcal{U}) \hookrightarrow \mathcal{H}_{G}^{\alpha} \stackrel{p^{\prime}}{\longrightarrow} \operatorname{Bun}_{G}$. Then $Y_{0}(\mathcal{U}) \rightarrow \mathcal{U}$ (respectively, $Y_{2}(\mathcal{U}) \rightarrow \mathcal{U}$ ) is a fibration with fibre isomorphic to $\mathbb{P}^{2 n-2}$ (respectively, to $\mathbb{A}^{2 n}$ ).

Let $Y_{k}(\widetilde{\mathcal{U}})$ be the preimage of $Y_{k}(\mathcal{U})$ in $\widetilde{\mathcal{H}}_{G}^{\alpha}$. For $k=0,2$ the restriction of the local system $\tilde{p}^{\prime *}\left({ }_{k}\right.$ Aut $) \otimes \kappa^{*} W$ descends under $Y_{k}(\widetilde{\mathcal{U}}) \rightarrow \widetilde{\mathcal{U}}$ to a local system, which is canonically identified, by Lemma 15 , with $\overline{\mathbb{Q}}_{\ell} \otimes_{1}$ Aut.

By Proposition 17, $\mathrm{H}\left(\mathcal{A}_{\alpha}, \mathrm{Aut}_{g}\right)$ vanishes over $X \times{ }_{0} \widetilde{\mathrm{Bun}}_{G}$, and we denote by $K$ the restriction of this complex to $\widetilde{\mathcal{U}}$. By decomposition theorem, $K$ is a direct sum of (shifted) irreducible perverse sheaves. We get an isomorphism

$$
\begin{aligned}
K \underset{\rightarrow}{ }{ }_{1} \operatorname{Aut}\left[d_{G}-2 n+1\right] & \left(\frac{d_{G}-2 n+1}{2}\right) \\
& \oplus_{1} \operatorname{Aut}\left[d_{G}+2 n-1\right]\left(\frac{d_{G}+2 n-1}{2}\right) \otimes \mathrm{R} \Gamma\left(\mathbb{P}^{2 n-2}, \overline{\mathbb{Q}}_{\ell}\right) .
\end{aligned}
$$

The first assertion follows.

(s) Set $\mathcal{V}=X \times{ }_{0} \mathrm{Bun}_{G}$. Let $K$ be the restriction of $\mathrm{H}\left(\mathcal{A}_{\alpha}, \mathrm{Aut}_{s}\right)$ to $\widetilde{\mathcal{V}}=X \times{ }_{0} \widetilde{\mathrm{Bun}}_{G}$. Let $Y(\mathcal{V})$ be the preimage of $\mathcal{V}$ under $\operatorname{supp} \times p: \mathcal{H}_{G}^{\alpha} \rightarrow X \times \operatorname{Bun}_{G}$. Write $Y_{k}(\mathcal{V})$ for the preimage of ${ }_{k} \operatorname{Bun}_{G}$ under $Y(\mathcal{V}) \hookrightarrow \mathcal{H}_{G}^{\alpha} \stackrel{p^{\prime}}{\longrightarrow} \operatorname{Bun}_{G}$. Then $Y_{1}(\mathcal{V}) \rightarrow \mathcal{V}$ is a fibration with fibre isomorphic to $\mathbb{P}^{2 n-1}$.

Let $Y_{1}(\widetilde{\mathcal{V}})$ be the preimage of $Y_{1}(\mathcal{V})$ in $\widetilde{\mathcal{H}}_{G}^{\alpha}$. By Lemma 15 , the *-restriction of $\tilde{p}^{\prime *}\left({ }_{1}\right.$ Aut $) \otimes \kappa^{*} W$ descends under $Y_{1}(\widetilde{\mathcal{V}}) \rightarrow \widetilde{\mathcal{V}}$ to a local system canonically identified with $\overline{\mathbb{Q}}_{\ell} \otimes_{0}$ Aut. By decomposition theorem, one gets an isomorphism

$$
K \widetilde{\rightarrow}_{0} \text { Aut } \otimes \mathrm{R} \Gamma\left(\mathbb{P}^{2 n-1}, \overline{\mathbb{Q}}_{\ell}\right)\left[d_{G}+2 n\right]\left(\frac{d_{G}+2 n}{2}\right) .
$$

We are done.

By decomposition theorem, $\mathrm{H}\left(\mathcal{A}_{\alpha}\right.$, Aut) is a direct sum of (shifted) irreducible perverse sheaves. Proposition 18 implies that $\mathrm{St}[1]\left(\frac{1}{2}\right) \otimes$ Aut appears in it as a direct summand. But 
according to Proposition 17, all the fibres of $\mathrm{H}\left(\mathcal{A}_{\alpha}\right.$, Aut $)$ and of $\mathrm{St}[1]\left(\frac{1}{2}\right) \otimes$ Aut are isomorphic. This concludes the proof of Theorem 4 .

\section{Acknowledgements}

I am very grateful to V. Drinfeld for his comments and suggestions about the first version of this paper. The idea of introducing the sheaf $S_{M}$ is due to him. I thank J.-L. Waldspurger for answering my questions and G. Laumon, D. Gaitsgory for stimulating discussions. I am also very grateful to the referee who has proposed so many improvements and corrections, in particular, the signs in the commutativity constraints (Proposition 14) have been clarified due to his comments.

\section{Appendix A}

A.1. For the convenience of the reader we collect here some generalities on group actions.

Let $f: \mathcal{Y} \rightarrow \mathcal{Z}$ be a morphism of stacks, $G \rightarrow \mathcal{Z}$ be a group scheme over $\mathcal{Z}$. Write $m_{G}$ for the product in $G$ and $1_{G}: \mathcal{Z} \rightarrow G$ for the unit section. Following [6], an action of $G$ on $\mathcal{Y}$ over $\mathcal{Z}$ is the data of a 1-morphism $m: G \times \mathcal{Z} \mathcal{Y} \rightarrow \mathcal{Y}$ over $\mathcal{Z}$, a 2-morphism $\mu: m \circ\left(m_{G} \times\right.$ id $) \Longrightarrow$ $m \circ($ id $\times m)$ making the following diagram 2-commutative

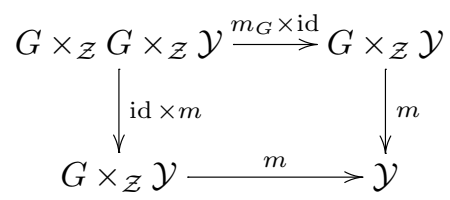

and a 2-morphism $\varepsilon: m \circ\left(1_{G} \times \mathrm{id}_{\mathcal{Y}}\right) \rightarrow \mathrm{id} \mathcal{y}$. They should satisfy two axioms: an associativity condition with respect to any 3 objects in $\mathrm{G}$ (cf. diagram (6.1.3) in loc.cit.); $\varepsilon$ is compatible with $\mu$ (cf. diagrams (6.1.4) in loc.cit.). The fact that $m$ is a $\mathcal{Z}$-morphism means that the diagram

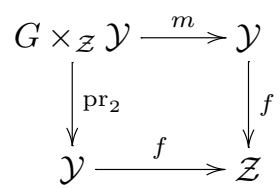

is 2 -commutative.

For a line bundle $L$ on $\mathcal{Y}$ we have a notion of $G$-equivariant structure on $L$ (cf. [17, Definition 2.8]). A version of this notion for an $\ell$-adic complex is as follows.

Definition 7. - A G-equivariant structure on $K \in \mathrm{D}(\mathcal{Y})$ is an isomorphism $\lambda: m^{*} K \overleftrightarrow{\longrightarrow}$ $\operatorname{pr}_{2}^{*} K$ such that two diagrams commute

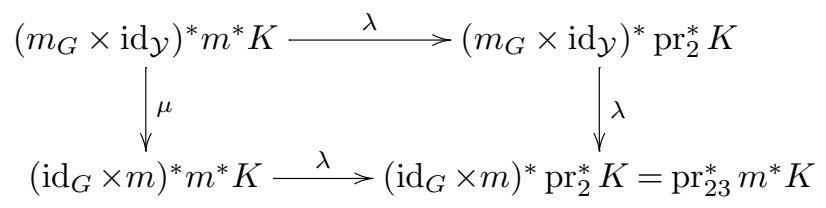

$4^{\mathrm{e}}$ SÉRIE - TOME $39-2006-\mathrm{N}^{\circ} 3$ 
and

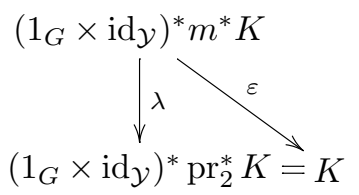

where $\operatorname{pr}_{2}: G \times_{\mathcal{Z}} \mathcal{Y} \rightarrow \mathcal{Y}$ and $\operatorname{pr}_{23}: G \times{ }_{\mathcal{Z}} G \times_{\mathcal{Z}} \mathcal{Y} \rightarrow G \times{ }_{\mathcal{Z}} \mathcal{Y}$ are the projections.

A.2. Let $f: \mathcal{Y} \rightarrow \mathcal{Z}$ be a representable morphism of algebraic stacks, $G \rightarrow \mathcal{Z}$ be a group scheme over $\mathcal{Z}$ acting on $\mathcal{Y}$ over $\mathcal{Z}$. By definition, $\mathcal{Y}$ is a $G$-torsor over $\mathcal{Z}$ if, locally in flat topology of $\mathcal{Z}, \mathcal{Y}$ is isomorphic to $G$ over $\mathcal{Z}$ as a $G$-scheme.

Assume that $\mathcal{Z}$ is locally of finite type. The notion of a perverse sheaf localizes in the smooth topology, so we have a notion of a perverse sheaf on $\mathcal{Z}$. For the same reason, if $G \rightarrow \mathcal{Z}$ is of finite type and smooth of relative dimension $d$ then the functor $K \mapsto f^{*} K[d]$ is an equivalence of the category of perverse sheaves $P(\mathcal{Z})$ on $\mathcal{Z}$ with the category of $G$-equivariant perverse sheaves $P_{G}(\mathcal{Y})$ on $\mathcal{Y}$.

A.3. Let $\mathcal{A}$ be a line bundle on a scheme $S$. Let $\widetilde{S} \rightarrow S$ denote the $\mu_{2}$-gerbe of square roots of $\mathcal{A}$ (cf. 3.3.1). Since $\mu_{2}$ acts on $\widetilde{S}$ by 2 -automorphisms of the identity id: $\widetilde{S} \rightarrow \widetilde{S}, \mu_{2}$ acts on any $K \in \mathrm{D}(\widetilde{S})$. Write $\pi: \widetilde{S} \rightarrow S$ for the structural morphism.

LEMMA 16. -

(1) The functor $\pi^{*}$ is an equivalence of the category of perverse sheaves on $S$ with the category of those perverse sheaves on $\widetilde{S}$ on which $\mu_{2}$ acts trivially.

(2) The functor $\pi^{*}: D(S) \rightarrow D(\widetilde{S})$ is fully faithful, its image $D_{+}(S)$ is a full triangulated subcategory of $D(\widetilde{S})$.

(3) For $K \in D(\widetilde{S})$ the following are equivalent:

(i) $-1 \in \mu_{2}$ acts as -1 on each cohomology sheaf of $K$,

(ii) $\pi_{!} K=0$,

(iii) $\pi_{*} K=0$.

Let $D_{-}(\widetilde{S}) \subset D(\widetilde{S})$ be the full triangulated subcategory of objects satisfying these conditions.

(4) For any $K_{ \pm} \in D_{ \pm}(\widetilde{S})$ we have $\operatorname{Hom}_{D(\widetilde{S})}\left(K_{+}, K_{-}\right)=0$ and $\operatorname{Hom}_{D(\widetilde{S})}\left(K_{-}, K_{+}\right)=0$.

For $K \in D(\widetilde{S})$ there exist $K_{ \pm} \in D_{ \pm}(\widetilde{S})$ such that $K \widetilde{\rightrightarrows} K_{+} \oplus K_{-}$.

Proof. - (1a) In the case $\mathcal{A}=\mathcal{O}_{S}$ consider the presentation $i: S \rightarrow B\left(S / \mu_{2}\right)$. The functor $i^{*}$ identifies the category of perverse sheaves on $B\left(S / \mu_{2}\right)$ with the category of perverse sheaves on $S$ equipped with an action of the group $\mu_{2}(S)$.

(1b) In general we have a Cartesian square

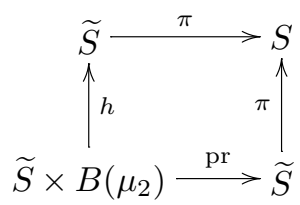

where $h$ sends a $T$-point $\left(\mathcal{B}, \mathcal{B}_{0},\left.\mathcal{B}^{2} \cong \mathcal{A}\right|_{T} \mathcal{B}_{0}^{2} \widetilde{\rightarrow} \mathcal{O}_{T}\right)$ to $\mathcal{B} \otimes \mathcal{B}_{0}$ for any $S$-scheme $T$.

If $F$ is a perverse sheaf on $\widetilde{S}$ on which $\mu_{2}$ acts trivially, then $\mu_{2} \times \mu_{2}$ acts trivially on $h^{*} F$. By (1a) we then get an isomorphism $h^{*} F \stackrel{\longrightarrow}{\longrightarrow} \mathrm{pr}^{*} F$ satisfying the usual cocycle condition. So, there is an isomorphism $F \stackrel{\rightarrow}{\rightarrow} \pi^{*} H$ for some perverse sheaf $H$ on $S$. 
(2) The map $\pi$ is smooth of relative dimension zero, and $\pi_{!} \overline{\mathbb{Q}}_{\ell} \widetilde{\rightarrow} \overline{\mathbb{Q}}_{\ell}$. It follows formally that $\pi^{*}$ is fully faithful.

(3) The functors $\pi_{!}$and $\pi_{*}$ are exact with respect to the usual t-structure. So, $\pi_{!} K=0$ iff $\pi_{!}\left(H^{i}(K)\right)=0$ for each $i$. The latter is equivalent to requiring that -1 acts nontrivially on $H^{i}(K)$ for each $i$. Similarly for $\pi_{*}$.

(4) Given $K_{-} \in D_{-}(\widetilde{S})$ and $K_{+} \widetilde{\rightarrow} \pi^{*} L \in D_{+}(\widetilde{S})$ we have

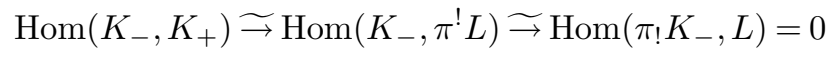

and

$$
\left.\operatorname{Hom}\left(K_{+}, K_{-}\right) \stackrel{\sim}{\rightarrow} \operatorname{Hom}\left(\pi^{*} L ; K_{-}\right) \stackrel{\operatorname{Hom}}{\rightarrow} L, \pi_{*} K_{-}\right)=0 .
$$

We claim that for each $K \in D(\widetilde{S})$ the adjointness map $\pi_{*} \pi^{*} \pi_{*} K \rightarrow \pi_{*} K$ is an isomorphism. Since our derived categories are bounded, by devissage we may assume that $K$ is placed in cohomological dimension zero. Then $K \stackrel{\Im}{\rightarrow} K_{0} \oplus K_{1}$, where -1 acts on $K_{0}$ (respectively, on $\left.K_{1}\right)$ as 1 (respectively, as -1 ). Clearly, $\pi^{*} \pi_{*} K_{0} \widetilde{\rightarrow} K_{0}$ and $\pi_{*} K_{1}=0$, so $\pi_{*} \pi^{*} \pi_{*} K \widetilde{\widetilde{S}} \pi_{*} K$.

For $K \in D(\widetilde{S})$ let $K_{-}$be a cone of the adjointness map $\pi^{*} \pi_{*} K \rightarrow K$ then $\pi_{*} K_{-}=0$. The triangle $\pi^{*} \pi_{*} K \rightarrow K \rightarrow K_{-}$splits, because $\operatorname{Hom}\left(K_{-}, \pi^{*} \pi_{*} K[1]\right)=0$.

Let $G$ be an algebraic group acting on $S$, assume that $\mathcal{A}$ is equipped with a $G$-equivariant structure. Then $G$ acts on $\widetilde{S}$, and the projection $\widetilde{S} \rightarrow S$ is $G$-equivariant.

The stack $\widetilde{S}$ is equipped with the universal line bundle $\mathcal{B}_{u}$ together with $\left.\mathcal{B}_{u}^{2} \widetilde{\rightarrow} \mathcal{A}\right|_{\widetilde{S}}$. One checks that $\mathcal{B}_{u}$ is $G$-equivariant.

Let $G$ act on the trivial gerbe $S \times B\left(\mu_{2}\right)$ as the product of the action of $G$ on $S$ with the trivial action on $B\left(\mu_{2}\right)$. The following lemma is straightforward.

LEMMA 17. - Let $\mathcal{B}$ be a $G$-equivariant line bundle on $S$ equipped with a $G$-equivariant isomorphism $\mathcal{B}^{2} \widetilde{\mathcal{A}}$. Then $\mathcal{B}$ yields a $G$-equivariant trivialization $\widetilde{S} \Im S \times B\left(\mu_{2}\right)$.

A.4. Let $S$ be a normal variety with a $\mathbb{G}_{m}$-action, $\mathcal{A}$ be a $\mathbb{G}_{m}$-equivariant line bundle on $S$. Write $\widetilde{S} \rightarrow S$ for the gerbe of square roots of $\mathcal{A}$. Let $S_{0} \subset S$ be the variety of fixed points. For a connected component $C$ of $S_{0}$ set

$$
S^{+}(C)=\left\{s \in S \mid \lim _{t \rightarrow 0} t s \in C\right\} \quad \text { and } \quad S^{-}(C)=\left\{s \in S \mid \lim _{t \rightarrow \infty} t s \in C\right\} .
$$

By [3], $S^{+}(C)$ is a locally closed subscheme of $S$ and the map $S^{+}(C) \rightarrow C$ sending $s$ to $\lim _{t \rightarrow 0} t s$ is a morphism of schemes (and similarly for $S^{-}(C)$ ).

Let $S^{+}$(respectively, $S^{-}$) denote the disjoint union of $S^{+}(C)$ (respectively, of $S^{-}(C)$ ) indexed by the connected components of $S_{0}$. Write $\widetilde{S}^{+}$(respectively, $\widetilde{S}^{-}, \widetilde{S}_{0}$ ) for the restriction of the gerbe $\widetilde{S} \rightarrow S$ to the corresponding scheme. Let $f^{ \pm}: \widetilde{S}_{0} \rightarrow \widetilde{S}^{ \pm}$and $g^{ \pm}: \widetilde{S}^{ \pm} \rightarrow \widetilde{S}$ denote the corresponding (representable) maps. Following [4], define hyperbolic localization functors $\mathrm{D}(\widetilde{S}) \rightarrow \mathrm{D}\left(\widetilde{S}_{0}\right)$ by

$$
K^{! *}=\left(f^{+}\right)^{!}\left(g^{+}\right)^{*} K, \quad K^{* !}=\left(f^{-}\right)^{*}\left(g^{-}\right)^{!} K .
$$

The following generalization of Theorem 1 from loc.cit. is straightforward.

Proposition 19. - There is a natural map $i_{S}: K^{* !} \rightarrow K^{! *}$ functorial in $K \in \mathrm{D}(\widetilde{S})$. Assume that there is a covering of $S$ by open $\mathbb{G}_{m}$-invariant subschemes $U_{i}$ and $\mathbb{G}_{m}$-invariant

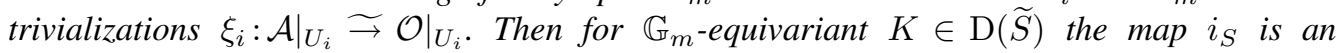
isomorphism.

$4^{\text {e }}$ SÉRIE - TOME $39-2006-\mathrm{N}^{\circ} 3$ 
Proof. - The map is constructed as in (loc.cit., Section 2). Let $\widetilde{U}_{i}$ denote the restriction of $\widetilde{S}$ to $U_{i}$. It suffices to show the desired map is an isomorphism over $\widetilde{U}_{i}$ for any perverse sheaf $K \in P(\widetilde{S})$. The trivialization $\xi_{i}$ induces $\mathbb{G}_{m}$-equivariant section $U_{i} \rightarrow \widetilde{U}_{i}$ of the gerbe $\widetilde{U}_{i} \rightarrow U_{i}$. One concludes applying Theorem 1 from loc.cit. for $\left.K\right|_{U_{i}}$.

Assume in addition that there is a $\mathbb{G}_{m}$-equivariant section $S^{+} \rightarrow \widetilde{S}^{+}$of the gerbe $\widetilde{S}^{+} \rightarrow S^{+}$. Let $h^{+}: S^{+} \rightarrow S_{0}$ be the map sending $s$ to $\lim _{t \rightarrow 0} t s$. Then for any $\mathbb{G}_{m}$-equivariant object

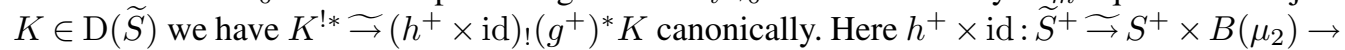
$S_{0} \times B\left(\mu_{2}\right)=\widetilde{S}_{0}$.

\section{Appendix B. Weil representation and the sheaf $S_{M}$}

B.1. Let $k=\mathbb{F}_{q}$ be a finite field with $q$ odd. Let $M$ be a symplectic space over $k$ of dimension $2 d$. The sheaf $S_{M}$ introduced in Section 4.4 has its origin in the Weil representation, this is what we are going to explain.

Consider the Heisenberg group $H(M)=M \oplus k$ with operation

$$
(m, a)\left(m^{\prime}, a^{\prime}\right)=\left(m+m^{\prime}, a+a^{\prime}+\frac{1}{2}\left\langle m, m^{\prime}\right\rangle\right)
$$

Fix an additive character $\psi: k \rightarrow \overline{\mathbb{Q}}_{\ell}^{*}$. There exists a unique up to isomorphism irreducible representation of $H(M)$ over $\overline{\mathbb{Q}}_{\ell}$ with central character $\psi$. Let $\left(\rho, S_{\psi}\right)$ be such representation. It yields an exact sequence

$$
1 \rightarrow \overline{\mathbb{Q}}_{\ell}^{*} \rightarrow \widetilde{G} \rightarrow G \rightarrow 1
$$

with $G=\operatorname{Sp}(M)$. Here

$$
\widetilde{G}=\left\{g, M[g] \mid g \in G, M[g] \in \operatorname{Aut} S_{\psi}, \rho(g m, a) \circ M[g]=M[g] \circ \rho(m, a)\right\} .
$$

Let $\mathcal{L}(M)$ denote the variety of Lagrangian subspaces of $M$. For $L \in \mathcal{L}(M)$ let $\chi_{L}: L \oplus k \rightarrow \overline{\mathbb{Q}}_{\ell}^{*}$ send $(l, a)$ to $\psi(a)$. Set

$$
S_{L, \psi}=\operatorname{Ind}_{L \oplus k}^{H(M)} \chi_{L}=\left\{f: H(M) \rightarrow \overline{\mathbb{Q}}_{\ell} \mid f(x h)=\chi_{L}(x) f(h) \text { for } x \in L \oplus k\right\} .
$$

For each $L \in \mathcal{L}(M)$ there is a pair $\left(v_{L} \in S_{\psi}, f_{L} \in S_{\psi}^{*}\right)$ which is $\left(L \oplus k, \chi_{L}\right)$-invariant. Normalize it by $f_{L}\left(v_{L}\right)=1$, so any such pair is $\left(a v_{L}, a^{-1} f_{L}\right)$ with $a \in \overline{\mathbb{Q}}_{\ell}^{*}$. Specifying such pair is equivalent to specifying an isomorphism of $H(M)$-modules $S_{\psi} \stackrel{\mathbb{\longrightarrow}}{\rightarrow} S_{L, \psi}$ such that the image of $f_{L}$ becomes the evaluation at zero $f_{L, s t} \in S_{L, \psi}^{*}$ (respectively, $v_{L}$ becomes the function $v_{L, s t}: H(M) \rightarrow \overline{\mathbb{Q}}_{\ell}$ supported at $L \oplus k$ with $\left.v_{L, s t}(0)=1\right)$.

Let $P_{L} \subset G$ be the Seigel parabolic subgroup preserving $L$. Restricting (37) we get an exact sequence

$$
1 \rightarrow \overline{\mathbb{Q}}_{\ell}^{*} \rightarrow \widetilde{P}_{L} \rightarrow P_{L} \rightarrow 1 .
$$

The action of $\widetilde{P}_{L}$ on $\overline{\mathbb{Q}}_{\ell} f_{L}$ yields a character $\widetilde{P}_{L} \rightarrow \overline{\mathbb{Q}}_{\ell}^{*}$ that splits this sequence (the group $\widetilde{P}_{L}$ acts on $\overline{\mathbb{Q}}_{\ell} v_{L}$ by the opposite character).

The finite-dimensional theta-function is $\theta_{L}: P_{L} \backslash \widetilde{G} / P_{L} \rightarrow \overline{\mathbb{Q}}_{\ell}$ given by $\theta_{L}(g)=f_{L}\left(g v_{L}\right)$, it does not depend on the choice of the pair $\left(v_{L}, f_{L}\right)$. 
B.2. Let $L_{1}, L_{2} \in \mathcal{L}(M)$. For $f \in S_{L_{1}, \psi}$ and $z \in L_{2} \oplus k$ the function $f(z h) \chi_{L_{2}}^{-1}(z)$ depends only on the image of $z$ in $L_{2}$, so we may set

$$
\left(F_{L_{1}, L_{2}}(f)\right)(h)=\int_{L_{2}} f(z h) \chi_{L_{2}}^{-1}(z) \mathrm{d} z,
$$

where $d z$ is the Haar measure on $L_{2}$ such that the volume of a point is one. Then $F_{L_{1}, L_{2}}: S_{L_{1}, \psi} \widetilde{\rightrightarrows}$ $S_{L_{2}, \psi}$ is an isomorphism of $H(M)$-modules.

One checks that $F_{L_{2}, L_{1}} \circ F_{L_{1}, L_{2}} \in \operatorname{Aut}\left(S_{L_{1}, \psi}\right)$ is the multiplication by $q^{d+\operatorname{dim}\left(L_{1} \cap L_{2}\right)}$.

Definition 8. - For $L_{1}, L_{2}, V \in \mathcal{L}(M)$ with $V \cap L_{i}=0$ define $\theta\left(L_{1}, L_{2}, V\right) \in \overline{\mathbb{Q}}_{\ell}^{*}$ by

$$
F_{L_{2}, L_{1}} \circ F_{V, L_{2}} \circ F_{L_{1}, V}=\theta\left(L_{1}, L_{2}, V\right) .
$$

We have $L_{1}=\left\{(b u+u) \mid u \in L_{2}\right\}$ for uniquely defined $b: L_{2} \rightarrow V$. The symplectic form on

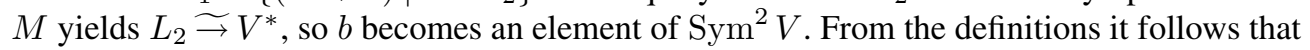

$$
\theta\left(L_{1}, L_{2}, V\right)=q^{d} \int_{V^{*}} \psi\left(\frac{1}{2}\left\langle b v^{*}, v^{*}\right\rangle\right) \mathrm{d} v^{*},
$$

where $d v^{*}$ is the Haar measure on $V^{*}$ such that the volume of a point is one.

Denote by $\widetilde{\mathcal{Y}}(k)$ the set of isomorphism classes of collections $L_{1}, L_{2} \in \mathcal{L}(M)$, a onedimensional space $\mathcal{B}$ together with $\mathcal{B}^{\otimes 2} \widetilde{\longrightarrow}\left(\operatorname{det} L_{1}\right) \otimes\left(\operatorname{det} L_{2}\right)$. So, $\widetilde{\mathcal{Y}}(k)$ is a two-sheeted covering of the set $\mathcal{Y}(k)$ of $G$-orbits on $\mathcal{L}(M) \times \mathcal{L}(M)$. Remind that $\mathcal{Y}(k)$ contains $d+1$ element.

Given a triple $L_{1}, L_{2}, V \in \mathcal{L}(M)$ with $L_{i} \cap V=0$, the form on $M$ yields isomorphisms

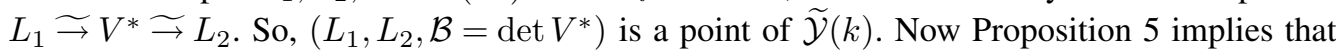
$\theta\left(L_{1}, L_{2}, V\right)$ depends only on the image of $\left(L_{1}, L_{2}, V\right)$ in $\widetilde{\mathcal{Y}}(k)$, so defining a function

$$
\theta: \tilde{\mathcal{Y}}(k) \rightarrow \overline{\mathbb{Q}}_{\ell}
$$

which is (up to a constant) the trace of Frobenius of the sheaf $S_{M}$. It is well-known that for $\left(L_{1}, L_{2}, \mathcal{B}\right) \in \widetilde{\mathcal{Y}}(k)$ with $i=\operatorname{dim}\left(L_{1} \cap L_{2}\right)$ one gets

$$
\theta\left(L_{1}, L_{2}, \mathcal{B}\right)^{2}=\left(\frac{-1}{q}\right)^{d-i} q^{3 d+i},
$$

where

$$
\left(\frac{-1}{q}\right)= \begin{cases}1, & \text { if }-1 \in k^{2} \\ -1, & \text { otherwise }\end{cases}
$$

B.3. Remind that we fixed a square root $q^{\frac{1}{2}}$ of $q$ in $\overline{\mathbb{Q}}_{\ell}$ (cf. 3.1). For $L_{1}, L_{2} \in \mathcal{L}(M)$ set

$$
\mathcal{F}_{L_{1}, L_{2}}=q^{\frac{1}{2}\left(-d-\operatorname{dim}\left(L_{1} \cap L_{2}\right)\right)} F_{L_{1}, L_{2}} .
$$

The following is a version of the Maslov index (cf. [18], appendix to Chapter 1).

Definition 9. - For $L_{1}, L_{2}, L_{3} \in \mathcal{L}(M)$ define $\gamma\left(L_{1}, L_{2}, L_{3}\right) \in \overline{\mathbb{Q}}_{\ell}^{*}$ by

$$
\mathcal{F}_{L_{2}, L_{1}} \circ \mathcal{F}_{L_{3}, L_{2}} \circ \mathcal{F}_{L_{1}, L_{3}}=\gamma\left(L_{1}, L_{2}, L_{3}\right) .
$$

$4^{\text {e }}$ SÉRIE - TOME $39-2006-\mathrm{N}^{\circ} 3$ 
Here are its immediate properties (cf. also loc.cit.).

PROPOSITION 20.-

(1) $\gamma\left(L_{1}, L_{2}, L_{3}\right)=\gamma\left(L_{1}, L_{3}, L_{2}\right)^{-1}=\gamma\left(L_{2}, L_{1}, L_{3}\right)^{-1}$.

(2) $\gamma\left(g L_{1}, g L_{2}, g L_{3}\right)=\gamma\left(L_{1}, L_{2}, L_{3}\right)$ for $g \in G$.

(3) If $L_{1}, L_{2}, L_{3}, L_{4} \in \mathcal{L}(M)$ then

$$
\gamma\left(L_{1}, L_{2}, L_{3}\right) \gamma\left(L_{1}, L_{4}, L_{2}\right)=\gamma\left(L_{3}, L_{4}, L_{2}\right) \gamma\left(L_{1}, L_{4}, L_{3}\right) .
$$

This implies that the function $\left(g_{1}, g_{2}\right) \mapsto \gamma\left(L, g_{1} L, g_{1} g_{2} L\right)$ is a 2-cocycle of $G$. This is the cocycle defining the extension (37). In our case of finite field $k$ this extension splits [21, Chapter 2, II.1].

\section{REFERENCES}

[1] Beauville A., Laszlo Y., Conformal blocks and generalized theta functions, Comm. Math. Phys. 164 (1994) 385-419.

[2] Beauville A., Laszlo Y., Un lemme de descente, C. R. Acad. Sci. Paris Série I 320 (1995) 335340.

[3] BiaŁYniCKI-Birula A., Some theorems on actions of algebraic groups, Ann. of Math. (2) 98 (1973) 480-497.

[4] BRADEN T., Hyperbolic localization of intersection cohomology, Transform. Groups 8 (3) (2003) 209-216.

[5] Braverman A., Gaitsgory D., Geometric Eisenstein series, Invent. Math. 150 (2002) 287-384.

[6] BREEN L., Bitorseurs et cohomologie non abélienne, in: The Grothendieck Festschrift, vol. 1, in: Progress in Math., vol. 86, Birkhäuser, Boston, 1990, pp. 401-476.

[7] Beilinson A., DRINFEld V., Quantization of Hitchin's integrable system and Hecke eigen-sheaves. Available at: http://www.math.uchicago.edu/ arinkin/langlands/.

[8] Deligne P., Le déterminant de la cohomologie, in: Current Trends in Arithmetical Algebraic Geometry, Arcata, CA, 1985, in: Contemp. Math., vol. 67, Amer. Math. Soc., Providence, RI, 1987, pp. 93-177.

[9] Deligne P., Letter to D. Kazhdan, 6 March 1985.

[10] Deligne P., Milne J.S., Tannakian categories, in: Hodge Cycles, Motives and Shimura Varieties, in: Lecture Notes in Math., vol. 900, Springer, Berlin, 1982.

[11] Drinfeld V., SyMPSON C., B-structures on G-bundles and local triviality, Math. Res. Letters 2 (1995) 823-829.

[12] Faltings G., Algebraic loop groups and moduli spaces of bundles, J. European Math. Soc. 5 (2003) 41-68.

[13] GAITSGORY D., Construction of central elements in the affine Hecke algebra via nearby cycles, Invent. Math. 144 (2) (2001) 253-280.

[14] GinZBURG V.A., Perverse sheaves on a Loop group and Langlands' duality, alg-geom/9511007.

[15] Howe R., $\theta$-series and invariant theory, Proc. Sympos. Pure Math., Part 133 (1979) 275-285.

[16] Laumon G., Transformation de Fourier homogène, Bull. Soc. Math. France 131 (4) (2003) 527-551.

[17] LASZLO Y., Linearization of group stack actions and the Picard group of the moduli of $\mathrm{SL}_{r} / \mu_{s^{-}}$ bundles on a curve, Bull. Soc. Math. France 125 (4) (1990) 529-545.

[18] LiOn G., Vergne M., The Weil Representation, Maslov Index and Theta Series, Progress in Math., vol. 6, Birkhäuser, Boston, 1980.

[19] MoORE C., Group extensions of $p$-adic and adelic linear groups, Publ. IHÉS 35 (1968) 5-70.

[20] Mirković I., ViLONEN K., Geometric Langlands duality and representations of algebraic groups over commutative rings, math.RT/0401222, Ann. Math., in press.

[21] Moeglin C., Vigneras M.-F., Waldspurger J.L., Correspondence de Howe sur un corps $p$-adique, Lecture Notes in Math., vol. 1291, Springer, Berlin, 1987. 
[22] PRASAD D., Weil Representation, Howe duality, and the Theta correspondence (lectures given in Montreal), http://www.mri.ernet.in/mathweb/dprasad.html.

[23] WEIL A., Sur certains groupes d'opérateurs unitaires, Acta Math. 111 (1964) 143-211.

(Manuscrit reçu le 6 juillet 2004; accepté, après révision, le 31 janvier 2006.)

\author{
Sergey LYSENKO \\ Institut de Mathématiques \\ Université Pierre et Marie Curie - Paris VI \\ 175 , rue du Chevaleret \\ 75013 Paris, France \\ E-mail: lysenko@math.jussieu.fr
}

